\title{
The ALMA Protostellar Interferometric Line Survey (PILS)
}

\section{First results from an unbiased submillimeter wavelength line survey of the Class 0 protostellar binary IRAS 16293-2422 with ALMA}

\author{
J. K. Jørgensen ${ }^{1}$, M. H. D. van der Wiel ${ }^{1}$, A. Coutens ${ }^{2}$, J. M. Lykke ${ }^{1}$, H. S. P. Müller ${ }^{3}$, E. F. van Dishoeck ${ }^{4,5}$, \\ H. Calcutt ${ }^{1}$, P. Bjerkeli ${ }^{1,6}$, T. L. Bourke ${ }^{7}$, M. N. Drozdovskaya ${ }^{4}$, C. Favre $^{8}$, E. C. Fayolle ${ }^{9}$, R. T. Garrod ${ }^{10}$, \\ S. K. Jacobsen ${ }^{1}$, K. I. Öberg ${ }^{9}$, M. V. Persson ${ }^{4}$, and S. F. Wampfler ${ }^{11}$ \\ ${ }^{1}$ Centre for Star and Planet Formation, Niels Bohr Institute \& Natural History Museum of Denmark, University of Copenhagen, \\ Øster Voldgade 5-7, 1350 Copenhagen K., Denmark \\ e-mail: jeskj@nbi.ku.dk \\ 2 Department of Physics and Astronomy, University College London, Gower St., London, WC1E 6BT, UK \\ 3 I. Physikalisches Institut, Universität zu Köln, Zülpicher Str. 77, 50937 Köln, Germany \\ ${ }^{4}$ Leiden Observatory, Leiden University, PO Box 9513, 2300 RA Leiden, The Netherlands \\ 5 Max-Planck Institut für Extraterrestrische Physik (MPE), Giessenbachstr. 1, 85748 Garching, Germany \\ ${ }^{6}$ Department of Earth and Space Sciences, Chalmers University of Technology, Onsala Space Observatory, 43992 Onsala, Sweden \\ 7 SKA Organization, Jodrell Bank Observatory, Lower Withington, Macclesfield, Cheshire SK11 9DL, UK \\ 8 Université Grenoble Alpes and CNRS, IPAG, 38000 Grenoble, France \\ 9 Harvard-Smithsonian Center for Astrophysics, 60 Garden Street, Cambridge, MA 02138, USA \\ 10 Departments of Chemistry and Astronomy, University of Virginia, Charlottesville, VA 22904, USA \\ 11 Center for Space and Habitability (CSH), University of Bern, Sidlerstrasse 5, 3012 Bern, Switzerland
}

Received 6 April 2016 / Accepted 28 July 2016

\begin{abstract}
Context. The inner regions of the envelopes surrounding young protostars are characterized by a complex chemistry, with prebiotic molecules present on the scales where protoplanetary disks eventually may form. The Atacama Large Millimeter/submillimeter Array (ALMA) provides an unprecedented view of these regions zooming in on solar system scales of nearby protostars and mapping the emission from rare species.

Aims. The goal is to introduce a systematic survey, the Protostellar Interferometric Line Survey (PILS), of the chemical complexity of one of the nearby astrochemical templates, the Class 0 protostellar binary IRAS 16293-2422, using ALMA in order to understand the origin of the complex molecules formed in its vicinity. In addition to presenting the overall survey, the analysis in this paper focuses on new results for the prebiotic molecule glycolaldehyde, its isomers, and rarer isotopologues and other related molecules.

Methods. An unbiased spectral survey of IRAS 16293-2422 covering the full frequency range from 329 to $363 \mathrm{GHz}(0.8 \mathrm{~mm})$ has been obtained with ALMA, in addition to a few targeted observations at 3.0 and $1.3 \mathrm{~mm}$. The data consist of full maps of the protostellar binary system with an angular resolution of $0.5^{\prime \prime}$ (60 AU diameter), a spectral resolution of $0.2 \mathrm{~km} \mathrm{~s}^{-1}$, and a sensitivity of 4-5 mJy beam ${ }^{-1} \mathrm{~km} \mathrm{~s}^{-1}$, which is approximately two orders of magnitude better than any previous studies.

Results. More than 10000 features are detected toward one component in the protostellar binary, corresponding to an average line density of approximately one line per $3 \mathrm{~km} \mathrm{~s}^{-1}$. Glycolaldehyde; its isomers, methyl formate and acetic acid; and its reduced alcohol, ethylene glycol, are clearly detected and their emission well-modeled with an excitation temperature of $300 \mathrm{~K}$. For ethylene glycol both lowest state conformers, $a G g^{\prime}$ and $g G g^{\prime}$, are detected, the latter for the first time in the interstellar medium (ISM). The abundance of glycolaldehyde is comparable to or slightly larger than that of ethylene glycol. In comparison to the Galactic Center these two species are over-abundant relative to methanol, possibly an indication of formation of the species at low temperatures in CO-rich ices during the infall of the material toward the central protostar. Both ${ }^{13} \mathrm{C}$ and the deuterated isotopologues of glycolaldehyde are detected, also for the first time ever in the ISM. For the deuterated species, a $\mathrm{D} / \mathrm{H}$ ratio of $\approx 5 \%$ is found with no differences between the deuteration in the different functional groups of glycolaldehyde, in contrast to previous estimates for methanol and recent suggestions of significant equilibration between water and - $\mathrm{OH}$ functional groups at high temperatures. Measurements of the ${ }^{13} \mathrm{C}$-species lead to $\mathrm{a}^{12} \mathrm{C}:{ }^{13} \mathrm{C}$ ratio of $\approx 30$, lower than the typical ISM value. This low ratio may reflect an enhancement of ${ }^{13} \mathrm{CO}$ in the ice due to either ion-molecule reactions in the gas before freeze-out or to differences in the temperatures where ${ }^{12} \mathrm{CO}$ and ${ }^{13} \mathrm{CO}$ ices sublimate.

Conclusions. The results reinforce the importance of low-temperature grain surface chemistry for the formation of prebiotic molecules seen here in the gas after sublimation of the entire ice mantle. Systematic surveys of the molecules thought to be chemically related, as well as the accurate measurements of their isotopic composition, hold strong promise for understanding the origin of prebiotic molecules in the earliest stages of young stars.
\end{abstract}

Key words. astrochemistry - stars: formation - stars: protostars - ISM: molecules - ISM: individual objects: IRAS 16293-2422 submillimeter: ISM 


\section{Introduction}

Understanding how, when, and where complex organic and potentially prebiotic molecules are formed is a fundamental goal of astrochemistry and an integral part of origins of life studies. The recent images from the Atacama Large Millimeter/submillimeter Array (ALMA) of a potentially planet-forming disk around a young star with an age of only $0.5-1$ Myr, HL Tau (ALMA Partnership et al. 2015), has highlighted the importance of the physics and chemistry of the early protostellar stages: How do stars evolve during their earliest evolutionary stages and, in particular, to what degree does the chemistry reflect this early evolution relative to, for example, the conditions in the environment in which the stars are forming? Already during its first years ALMA has demonstrated enormous potential for addressing these issues with its high angular resolution and sensitivity making it possible to zoom in on young stars on solar system scales and map the chemical complexity in their environments (e.g., Pineda et al. 2012; Jørgensen et al. 2012, 2013; Persson et al. 2013; Codella et al. 2014; Sakai et al. 2014; Friesen et al. 2014; Lindberg et al. 2014; Oya et al. 2014; Murillo et al. 2015; Podio et al. 2015; Belloche et al. 2016; Müller et al. 2016).

A particular focus of ALMA observations is the search for complex molecules in regions of low-mass star formation. Over the last decade it has become clear that the chemical complexity toward the innermost envelopes of solar-type protostars can rival that of more massive hot cores (see, e.g., review by Herbst \& van Dishoeck 2009). Complex molecules are not solely attributed to such regions, but are also found toward cold prestellar cores (e.g., Öberg et al. 2010; Bacmann et al. 2012; Vastel et al. 2014) and toward outflow driven shocks (e.g., Arce et al. 2008; Sugimura et al. 2011; Mendoza et al. 2014). The big questions that remain include; (a) what degree of molecular complexity can arise during the protostellar stages; (b) how exactly do complex organics form; (c) what are the roles of grain-surface/ice-mantle vs. gas-phase reactions at low and high temperatures for specific molecules; and (d) what is the importance of external conditions (e.g., cosmic ray induced ionization, UV radiation) and the physical environment (e.g., temperature)?

Many of these questions can potentially be addressed through systematic surveys with ALMA: with its high angular resolution we can zoom in on the smallest scales toward young stars making it possible to unambiguously identify the emitting regions for different molecules. The advantage of studying the hot inner regions of the envelopes around protostars is that the ices there are fully sublimated and all the molecules are present in the gas-phase. ALMA's high sensitivity and spectral resolution allows for identification of faint lines of rare species and also observations of more quiescent sources (e.g., of lower masses) for which line confusion is reached at a much deeper level than for sources of higher masses. One particularly interesting source in this context is the well-studied protostellar binary, IRAS 16293-2422, the first low-mass protostar for which complex organic molecules (van Dishoeck et al. 1995; Cazaux et al. 2003) and prebiotic species (Jørgensen et al. 2012) were identified - the latter already during ALMA science verification.

This paper presents an overview of an unbiased survey, the Protostellar Interferometric Line Survey (PILS) ${ }^{1}$ of IRAS 16293-2422 with ALMA covering a wide frequency window from 329 to $363 \mathrm{GHz}$ at $0.5^{\prime \prime}$ angular resolution $(60 \mathrm{AU}$ diameter), and also other selected frequencies around $1.3 \mathrm{~mm}$ $(230 \mathrm{GHz})$ and $3.0 \mathrm{~mm}(100 \mathrm{GHz})$. The paper provides a first

\footnotetext{
1 http://youngstars.nbi.dk/PILS
}

overview of the observations and data and presents new results concerning the presence of glycolaldehyde and related species, as well as the first detections of its rarer ${ }^{13} \mathrm{C}$ and deuterated isotopologues. The paper is laid out as follows. Section 2 presents a detailed review of studies of the physics and chemistry of IRAS 16293-2422 as background for this and subsequent PILS papers and Sect. 3 presents an overview of the details of the observations and reduction. Section 4 presents the overall features of the datasets including the continuum emission at the three different wavelengths and information about the line emission, while Sect. 5 focuses on the analysis of the emission from glycolaldehyde and related molecules (Sect. 5.2) and its isotopologues (Sect. 5.3) with particular emphasis on the constraints on formation scenarios for these species. Section 6 summarizes the main findings of the paper.

\section{Overview of IRAS 16293-2422}

IRAS 16293-2422 (hereafter IRAS 16293) is a deeply embedded young stellar object located in the L1689 region in the eastern part of the $\rho$ Ophiuchus cloud complex studied extensively through larger scale infrared and submillimeter continuum and line maps (e.g., Tachihara et al. 2000; Nutter et al. 2006; Young et al. 2006; Jørgensen et al. 2008; Padgett et al. 2008). The traditionally quoted distance for $\rho$ Oph was $\approx 160 \mathrm{pc}$ (Whittet \& van Breda 1975). However, most recent distance estimates for the bulk of the $\rho$ Oph cloud complex place it nearer, at approximately $120 \mathrm{pc}$ based on extinction measurements (Knude \& Høg 1998; Lombardi et al. 2008) and VLBI parallax measurements (Loinard et al. 2008). One dedicated measurement of the parallax of water masers toward IRAS 16293-2422 place it at a much larger distance than the rest of the cloud $(\approx 178$ pc), but Rivera et al. (2015) argue that this estimate may be hampered by the weak and highly variable nature of water masers and quote new measurements that place IRAS 16293-2422 at the nearer distance. We therefore adopt a distance of $120 \mathrm{pc}$ throughout this paper. For this distance, the observed luminosity of the source is $21 \pm 5 L_{\odot}$ estimated by pure integration of its SED with data from the mid-infrared from Spitzer (Jørgensen et al. 2005b), far-infrared from ISO-LWS (Correia et al. 2004) and Herschel/SPIRE (Makiwa 2014), and submillimeter (Schöier et al. 2002). To put the new ALMA PILS results into context, the following presents an overview of physical and chemical studies of this source to date.

\subsection{Physics}

Much of our current picture for star formation goes back to the 1980s when infrared observations (in particular, the full survey of the infrared sky by the Infrared Astronomical Satellite) gave rise to the identification and empirical classification of young stellar objects (Lada \& Wilking 1984) and combined with theoretical work on the collapse of dense cores and the formation of disks (e.g., Terebey et al. 1984; Adams \& Shu 1986) led to the generally accepted picture of the evolution of young stellar objects (Shu et al. 1987). One of the particular goals of these studies was to identify the youngest protostars and IRAS 16293-2422 with its very red colors in that context became a particularly interesting target for immediate follow-up studies using ground-based millimeter wavelength single-dish telescopes and small interferometers. The focus of these early line studies was to address whether the circumstellar material was predominantly characterized by infall toward the protostar 
(Walker et al. 1986) or rather by rotation in a disk-like structure (Mundy et al. 1986; Menten et al. 1987).

André et al. (1993) introduced the term "Class 0" protostar for young stellar objects thought to have accreted less than half of their final mass and still deeply embedded in their protostellar envelopes. André et al. noted that IRAS 16293-2422 was a candidate of such a source. Its very strong submillimeter emission made it unlikely that it was simply an edge-on Class I object, but instead a very young, still deeply embedded, protostar. The distribution of the dust around the source was recognized early on as being consistent with originating in a power-law density profile envelope (Walker et al. 1990) following $\rho \propto r^{-p}$ with $p \approx 1.5-2.5$ as theoretically predicted for a singular-isothermal sphere or free-falling envelope. Ceccarelli et al. (2000a), Schöier et al. (2002), and others have modeled the lower resolution single-dish observations of IRAS 16293-2422 and found that such envelope profiles reproduce far-infrared/(sub)millimeter line and continuum observations, and that such models thereby could be taken as a useful reference, for example, to describe the chemistry at larger scales ( $\gtrsim 1000 \mathrm{AU})$. Mapping of the protostellar envelope with singledish telescopes demonstrated that the material could be characterized by infall on a few thousand AU scales (Zhou 1995; Narayanan et al. 1998), although more quantitative statements are complicated owing to the underlying physical structure that appears at higher angular resolution as multiple separated velocity components (e.g., Schöier et al. 2004).

On smaller scales, IRAS 16293-2422 was the first protostar identified as a binary, being resolved into two separate components, IRAS 16293A and IRAS 16293B, at radio (Wootten 1989) and millimeter (Mundy et al. 1992) wavelengths with a separation of $\approx 5.1^{\prime \prime}$ or 620 AU (Looney et al. 2000; Chandler et al. 2005). Each of the two components shows compact millimeter continuum emission on $100 \mathrm{AU}$ scales, signs of two protostars each surrounded by a compact disk-like structure embedded within a larger circumbinary envelope (Looney et al. 2000; Schöier et al. 2004). The early observations demonstrated that the continuum fluxes toward the two components have different dependencies on wavelength, with IRAS 16293A showing a flattened continuum spectrum at wavelengths longer than $3 \mathrm{~mm}$, while IRAS 16293B has a continuum spectrum that is accurately described by a single power-law, $F_{v} \propto v^{\alpha}$, with $\alpha=2.0-2.5$ from $\mathrm{cm}$ through submillimeter wavelengths (Mundy et al. 1992; Schöier et al. 2004; Chandler et al. 2005). A plausible explanation for this difference is that the emission from IRAS $16293 \mathrm{~A}$ is a combination of dust continuum radiation with shock-ionized emission at longer wavelengths (Chandler et al. 2005), while IRAS $16293 \mathrm{~B}$ is dominated by optically thick dust continuum emission. IRAS 16293A is in fact resolved into multiple components at longer wavelengths (Wootten 1989; Chandler et al. 2005; Loinard et al. 2007; Pech et al. 2010) and possibly itself a tight binary within a distance of about $1^{\prime \prime}$ (120 AU). The early claims of rotation in a "disk" encompassing IRAS 16293A and IRAS 16293B were likely the result of a difference in the LSR velocities of the two sources (3.1 vs. $2.7 \mathrm{~km} \mathrm{~s}^{-1}$; Jørgensen et al. 2011) for which the physical origin is still not fully understood. IRAS 16293A does show a small velocity gradient in the NE-SW direction close to the location of the continuum peak that could be attributed to rotation in a disk located in this direction (Pineda et al. 2012; Girart et al. 2014), although the velocity profile of this component is only consistent with Keplerian rotation if the dynamical mass of IRAS 16293A is very small $\left(\sim 0.1 M_{\odot}\right.$; Favre et al. 2014b). Also, the relative contributions to the overall luminosity of IRAS 16293-2422 from the different sources remain unclear.

The binarity is also reflected in the complex outflow morphologies observed toward the system. The outflow structure has been studied in different tracers and at very different angular resolutions from its first detections (Fukui et al. 1986; Wootten \& Loren 1987). It was recognized early on as being quadrupolar in nature (Walker et al. 1988; Mizuno et al. 1990) with one collimated pair of lobes in the NE-SW direction and one less collimated (and less well-aligned set of lobes) in the E-W direction. This complicated outflow morphology has led to some discussion concerning the nature of IRAS 16293A and IRAS 16293B: it has long been accepted that IRAS 16293A is of protostellar nature and the NE-SW outflow driven by this source (e.g., Castets et al. 2001; Stark et al. 2004). However, the lack of clear outflow structures associated with IRAS 16293B itself has made its nature more ambiguous: Stark et al. (2004) suggested that IRAS 16293B was in fact a more evolved T Tauri star responsible for the "fossil" outflow in the E-W direction, but higher resolution $\mathrm{CO}$ images from the SMA showed that a compact outflow in the $\mathrm{E}-\mathrm{W}$ direction originates close to IRAS 16293A, possibly the current manifestation of the larger scale, less collimated, E-W flow (Yeh et al. 2008). Also, direct detections of infall toward the source (Chandler et al. 2005; Pineda et al. 2012; Jørgensen et al. 2012) suggest that it is in fact in an early evolutionary stage. Based on ALMA CO 6-5 images, Loinard et al. (2013) argued that blue-shifted emission seen to the southeast of IRAS 16293B (see also Yeh et al. 2008; and Jørgensen et al. 2011) was the manifestation of a very young (mono-polar) outflow from IRAS 16293B. Kristensen et al. (2013), however, used the same data to argue that it was instead a bow-shock associated with a (new) NW-SE outflow driven from IRAS 16293A.

Finally, larger scale maps reveal a companion core to that associated with IRAS 16293-2422 itself, IRAS 16293E, offset by about $1.5^{\prime}$. This core was first recognized as a prominent $\mathrm{NH}_{3}$ core (Mizuno et al. 1990) and is almost as bright in submillimeter continuum as the core hosting IRAS 16293-2422 itself (e.g., Nutter et al. 2006). Castets et al. (2001) mapped the region in $\mathrm{H}_{2} \mathrm{CO}, \mathrm{N}_{2} \mathrm{H}^{+}$, and other tracers using single-dish telescopes and suggested that the source was in fact also a Class 0 protostar driving a separate outflow in the northwest-southeast direction. However, Stark et al. (2004) instead argued that this was the reflection of the interaction between the outflow driven by IRAS 16293-2422 with the dense IRAS $16293 \mathrm{E}$ core, itself therefore likely a dense prestellar core. In support of this interpretation, Jørgensen et al. (2008) did not find any signs of an embedded protostar toward IRAS 16293E through Spitzer mid-infrared observations, but rather extended $4.5 \mu \mathrm{m}$ emission (molecular hydrogen) originating toward IRAS 16293-2422 and extending to and around IRAS 16293E.

\subsection{Chemistry}

\subsubsection{Single-dish observations}

IRAS 16293 has a particularly rich molecular line spectrum for a low-mass protostar, as has been recognized since the late 1980s. Given the limited sensitivity of early interferometers, chemical studies were mainly performed with single-dish telescopes, especially the then newly available Caltech Submillimeter Observatory (CSO) and James Clerk Maxwell Telescope (JCMT) equipped with sensitive submillimeter receivers and located on Mauna Kea, a high and dry site with easy access to this southern source (van Dishoeck et al. 1993). Individual line settings in the 
230 and $345 \mathrm{GHz}$ windows revealed 265 lines belonging to 24 different molecules (Blake et al. 1994; van Dishoeck et al. 1995). Because of its rich spectrum, IRAS 16293 was quickly dubbed the low-mass counterpart of Orion-KL. Through rotational diagrams, non-LTE excitation calculations and line profile analyses, at least three different physical and chemical components were identified: a compact, turbulent, warm $(>80 \mathrm{~K})$, and dense $\left(\sim 10^{7} \mathrm{~cm}^{-3}\right)$ region rich in $\mathrm{Si}$ - and $\mathrm{S}$-bearing molecules and complex organic molecules such as $\mathrm{CH}_{3} \mathrm{OH}$ and $\mathrm{CH}_{3} \mathrm{CN}$; the quiescent circumbinary envelope best traced in common molecules like $\mathrm{CS}, \mathrm{HCO}^{+}$, and $\mathrm{H}_{2} \mathrm{CO}$; and a colder outer envelope and surrounding cloud core seen in radicals such as $\mathrm{CN}$, $\mathrm{C}_{2} \mathrm{H}$, and $\mathrm{C}_{3} \mathrm{H}_{2}$ with very narrow lines.

These early data also revealed that IRAS 16293 has very high abundances of deuterated molecules, with $\mathrm{C}_{2} \mathrm{D} / \mathrm{C}_{2} \mathrm{H}$ and $\mathrm{HDCO} / \mathrm{H}_{2} \mathrm{CO}$ values $>0.1$. This extreme fractionation is thought to be a result of gas-grain chemistry, benefitting from much longer timescales at very low temperatures and high densities compared with high-mass sources (van Dishoeck et al. 1995). Subsequent deeper observations revealed that doubly and even triply deuterated molecules are common toward IRAS 16293 and the nearby cold clump IRAS 16293E: $\mathrm{D}_{2} \mathrm{CO}$ (Ceccarelli et al. 1998a; Loinard et al. 2000), $\mathrm{ND}_{2} \mathrm{H}$ (Loinard et al. 2001; Lis et al. 2006; Gerin et al. 2006), $\mathrm{ND}_{3}$ (Roueff et al. 2005), $\mathrm{D}_{2} \mathrm{O}$ (Butner et al. 2007; Vastel et al. 2010), $\mathrm{CHD}_{2} \mathrm{OH}$ (Parise et al. 2002), and $\mathrm{CD}_{3} \mathrm{OH}$ (Parise et al. 2004). The extreme deuteration of $\mathrm{D}_{2} \mathrm{CO}$ over scales of a few thousand $\mathrm{AU}$ was considered to be the smoking gun that proved active grain surface chemistry (Ceccarelli et al. 2001). Around the same time, the detection of $\mathrm{H}_{2} \mathrm{D}^{+}$in protostellar sources including IRAS 16293 (Stark et al. 1999, 2004) and prestellar cores (Caselli et al. 2003) pointed toward enhanced abundances of gaseous $\mathrm{H}_{2} \mathrm{D}^{+}, \mathrm{D}_{2} \mathrm{H}^{+}$, and even $\mathrm{D}_{3}^{+}$in cold cores assisted by heavy freeze-out of $\mathrm{CO}$, which in turn drive high deuteration fractions of other gas-phase species (Roberts \& Millar 2000; Roberts et al. 2003). The doubly-deuterated isotopologue $\mathrm{D}_{2} \mathrm{H}^{+}$ was indeed first detected toward IRAS 16293E by Vastel et al. (2004).

Another astrochemical milestone came from highly sensitive IRAM $30 \mathrm{~m}$ spectra revealing a much wider variety of complex organic molecules than found in early data, including $\mathrm{HCOOCH}_{3}, \mathrm{CH}_{3} \mathrm{OCH}_{3}$, and $\mathrm{C}_{2} \mathrm{H}_{5} \mathrm{CN}$ (Cazaux et al. 2003). Subsequent full spectral scans at $3,2,1$, and $0.9 \mathrm{~mm}$ with the IRAM $30 \mathrm{~m}$ and JCMT as part of the TIMASSS survey (Caux et al. 2011) detected thousands of lines belonging to simple and complex species (Jaber et al. 2014), including the first detection of an amide, $\mathrm{NH}_{2} \mathrm{CHO}$ (formamide), in a low-mass protostar (Kahane et al. 2013). An early search for the simplest amino acid, glycine, was unsuccessful (Ceccarelli et al. 2000b). IRAS 16293 has also been surveyed with the HIFI instrument on the Herschel Space Observatory in various bands between $460 \mathrm{GHz}$ and $2 \mathrm{THz}$ (Ceccarelli et al. 2010; Hily-Blant et al. 2010; Bacmann et al. 2010; Bottinelli et al. 2014) and by the GREAT instrument on SOFIA (Parise et al. 2012), revealing various (deuterated) hydrides, water lines (see below), and highexcitation lines of heavier molecules.

Water is clearly abundant near IRAS 16293, revealed originally by maser emission at radio wavelengths (Wootten 1989; Furuya et al. 2001) and subsequently by thermal emission using the Infrared Space Observatory (ISO; Ceccarelli et al. 1998b, 1999) and most recently with the Herschel Space Observatory (Ceccarelli et al. 2010; Coutens et al. 2012). Herschel observed lines over a wide energy range and from minor isotopologues, $\mathrm{H}_{2}^{18} \mathrm{O}$ and $\mathrm{H}_{2}^{17} \mathrm{O}$, which are less dominated by the strong outflow emission than $\mathrm{H}_{2}^{16} \mathrm{O}$. Several lines of deuterated water, HDO (Coutens et al. 2012) and $\mathrm{D}_{2} \mathrm{O}$ (Vastel et al. 2010; Coutens et al. 2013) were detected as well.

Determination of abundances from this wealth of data has been hindered by the realization that there are gradients in temperature, density, and chemistry throughout the source. Early analyses hinted at a jump in abundances of organic molecules in the inner envelope by up to two orders of magnitude taking into account the beam dilution of the inner warm region in the large observing beam (van Dishoeck et al. 1995). Quantitative abundance determinations with varying temperature and density profiles using jump abundance models were subsequently introduced when non-LTE excitation and radiative transfer codes became available (van der Tak et al. 2000; Ceccarelli et al. 2000a; Schöier et al. 2002; Ceccarelli et al. 2003; Doty et al. 2004). The jump in abundance was typically put at dust temperatures around $100 \mathrm{~K}$ when water ice sublimates, together with any species embedded in the ice (Fraser et al. 2001), although $\mathrm{H}_{2} \mathrm{CO}$ was recognized to return to the gas at lower temperatures, around 50 K (Ceccarelli et al. 2001; Schöier et al. 2004), and CO at even lower temperatures, around $25 \mathrm{~K}$ (Jørgensen et al. 2002). This inner region with temperatures above $100 \mathrm{~K}$ is also called the hot core or hot corino. The realization of varying abundances also pointed to another conundrum, namely that optically thin $\mathrm{C}^{18} \mathrm{O}$ emission could not be used as a tracer of the $\mathrm{H}_{2}$ column density since the emission arises from a much larger area than that of the organic molecules. This is one of the main reasons for the large discrepancies in derived abundances that are scattered throughout the literature (see Herbst \& van Dishoeck 2009 , for further details). For molecules with a sufficient number of observed lines originating from a range of upper energy levels, inner and outer envelope abundances can be determined within the context of the adopted physical model (Ceccarelli et al. 2000c; Schöier et al. 2002; Maret et al. 2004, 2005; Jørgensen et al. 2005c; Coutens et al. 2012). In the systematic search for complex organic molecules in the TIMASSS survey, it was found that a number of species, including ketene, acetaldehyde, formamide, dimethyl ether, and methyl formate, show emission not just from the inner hot core, but also from the cold extended envelope probed by the single-dish observations (Jaber et al. 2014).

The early data also triggered the debate of the importance of thermal sublimation of ices in the inner hot core region versus shocks associated with the outflows. The broad line profiles of $\mathrm{Si}$ - and S-bearing molecules, as well as some complex organic molecules like $\mathrm{CH}_{3} \mathrm{OH}$ and $\mathrm{CH}_{3} \mathrm{CN}$, hinted at the importance of sputtering along the outflow walls, much like that seen for $\mathrm{SiO}$ and $\mathrm{CH}_{3} \mathrm{OH}$ at outflow spots away from the protostar in this and other sources (e.g., Bachiller \& Pérez Gutiérrez 1997; Hirano et al. 2001; Garay et al. 2002; Jørgensen et al. 2004). These lines are clearly broader than can be explained by an infalling envelope. A particularly important molecule in the debate of the importance of shocks versus quiescent hot core emission is water itself, with both origins being argued in the literature (Ceccarelli et al. 2000a; Nisini et al. 2002). New data on optically thin isotopologues, spatially resolved interferometry by Persson et al. (2013), and data such as is presented here, provide evidence for both scenarios.

\subsubsection{Interferometric observations}

Early interferometer observations revealed elongated $\mathrm{C}^{18} \mathrm{O}$ and CS emission coincident with the dust distributions on $\sim 800 \mathrm{AU}$ scales, with $\mathrm{NH}_{3}$ arising from a larger (8000 AU diameter) 
region (Mundy et al. 1990; Walker et al. 1990). Also, very strong SO emission was found centered on IRAS 16293A, but not on IRAS 16293B, providing early evidence for chemical differentiation between the two sources (Mundy et al. 1992).

Once interferometers grew from three to six telescopes and the Submillimeter Array (SMA) was inaugurated, IRAS 16293 became a prime target for spatially resolved astrochemical studies. The first arcsecond resolution images by Bottinelli et al. (2004b) and Kuan et al. (2004) demonstrated that the emission from complex organic molecules peak at the locations of the components of the protostellar binary on scales argued to be consistent with thermal evaporation. Both studies showed some evidence for differences between the intensities of lines of different species toward the two sources, but with the emission toward IRAS 16293A generally being brighter. A systematic interferometric study (Bisschop et al. 2008; Jørgensen et al. 2011) demonstrated the association of some nitrogen-containing organic molecules $\left(\mathrm{HNCO}, \mathrm{CH}_{3} \mathrm{CN}\right)$ primarily with IRAS 16293A, while a number of oxygencontaining species are present with comparable intensities toward both sources and one, acetaldehyde $\left(\mathrm{CH}_{3} \mathrm{CHO}\right)$, almost exclusively associated with IRAS $16293 \mathrm{~B}$. When normalized to the column densities of methanol, these differences translate into relative abundance differences of up to an order of magnitude between different species toward the two sources (Bisschop et al. 2008). Subarcsecond resolution observations by Chandler et al. (2005) indicated that the emission from molecules characterized by high rotation temperatures peak toward one of the shock positions in the vicinity of IRAS 16293A rather than the protostar itself. The presence of shocks on small scales toward IRAS $16293 \mathrm{~B}$ is demonstrated by high angular resolution images of $\mathrm{SiO}$ (Jørgensen et al. 2011), with some evidence for the importance of shocks on other species (including water on larger scales). Recently, Oya et al. (2016) analyzed the kinematics around source $\mathrm{A}$ and found evidence for chemical differentiation between OCS on the one hand and $\mathrm{CH}_{3} \mathrm{OH}$ and $\mathrm{CH}_{3} \mathrm{OCHO}$ on the other with the latter emission more compact, possibly associated with weak shocks at the centrifugal barrier of the disk forming around IRAS 16293A.

Despite the natural focus of the interferometric studies on the warm compact gas, the observations also demonstrated the importance of the colder parts of the protostellar environments. For example, observations of lower excited $\mathrm{H}_{2} \mathrm{CO}$ (Schöier et al. 2004) and HNCO (Bisschop et al. 2008) transitions show that significant abundance (and optical depth) variations are present in the colder gas in the circumbinary envelope. The images also demonstrated spatial differences between species such as $\mathrm{C}^{18} \mathrm{O}$, $\mathrm{DCO}^{+}$, and $\mathrm{N}_{2} \mathrm{D}^{+}$in the colder gas that can be explained through relatively simple gas-phase chemistry (Jørgensen et al. 2011).

The chemical richness (and differentiations) of the gas toward IRAS 16293, seen in the 6-8 element interferometric data, made it an obvious target for science verification observations with ALMA at $220 \mathrm{GHz}$ or $1.4 \mathrm{~mm}$ (Pineda et al. 2012; Jørgensen et al. 2012) and at $690 \mathrm{GHz}$ or $0.43 \mathrm{~mm}$ (Baryshev et al. 2015). These early observations were already more than an order of magnitude more sensitive than the previous interferometric data and, as mentioned above, showed the first discovery of a prebiotic molecule, glycolaldehyde, toward a solar-type protostar (Jørgensen et al. 2012). Additional science verification observations at $690 \mathrm{GHz}(0.4 \mathrm{~mm})$ produced images of one line of the $\mathrm{H}_{2}^{18} \mathrm{O}$ water isotopologue. Together with observations of both $\mathrm{H}_{2}^{18} \mathrm{O}$ and HDO from the Submillimeter Array, these measurements constrained the $\mathrm{HDO} / \mathrm{H}_{2} \mathrm{O}$ abundance ratio to $9 \times 10^{-4}$ (Persson et al. 2013), much lower than the value in the colder parts of the envelope (e.g., Coutens et al. 2012) and more in line with the ratios for Earth's oceans and solar system comets.

\section{Observations}

IRAS 16293-2422 was observed as part of the Protostellar Interferometric Line Survey (PILS) program (PI: Jes K. Jørgensen). The survey consists of an unbiased spectral survey covering a significant part of ALMA's Band 7 (wavelengths of approximately $0.8 \mathrm{~mm}$ ) in ALMA's Cycle 2 (projectid: 2013.1.00278.S) as well as selected windows in ALMA's Bands $3(\approx 3 \mathrm{~mm}$, or $100 \mathrm{GHz})$ and $6(\approx 1.3 \mathrm{~mm}$, or $230 \mathrm{GHz})$ obtained in Cycle 1 (project-id: 2012.1.00712.S). The following sections describe each of these datasets ${ }^{2}$ and the reduction process.

\subsection{Band 7 data: $329-363 \mathrm{GHz}$ unbiased line survey}

\subsubsection{Data, calibration, and imaging}

The Band 7 part of the survey covers in full the frequency range from $329.147 \mathrm{GHz}$ to $362.896 \mathrm{GHz}$. Data were obtained from both the array of $12 \mathrm{~m}$ dishes (typically 36-41 antennas in the array at the time of observations) and the Atacama Compact Array (ACA), or Morita Array, of $7 \mathrm{~m}$ dishes (typically 8-10 antennas). Table 1 presents a full log of the Band 7 observations. The pointing center in both cases was set to be a location between the two components of the binary system at $\alpha_{\mathrm{J} 2000}=16^{\mathrm{h}} 32^{\mathrm{m}} 22 \mathrm{~s} .72$; $\delta_{\mathrm{J} 2000}=-24^{\circ} 28^{\prime} 34^{\prime \prime}$.3. In total 18 spectral settings were observed: each setting covers a bandwidth of $1875 \mathrm{MHz}$ (over four different spectral windows, $468.75 \mathrm{MHz}$ wide). To limit the data rate the data were downsampled by a factor of two relative to the native spectral resolution of the array, resulting in a spectral resolution of $0.244 \mathrm{MHz}\left(\approx 0.2 \mathrm{~km} \mathrm{~s}^{-1}\right)$ over 1920 channels for each spectral window. Each setting was observed with approximately 13 min integration on source (execution blocks of approximately 40 min including calibrations) for the $12 \mathrm{~m}$ array and double that for the ACA.

The calibration and imaging proceeded according to the standard recipes in CASA. Titan and, in a few instances, Ceres were used as flux calibrators, while the quasar J1517-2422 $(\approx 1.2 \mathrm{Jy})$ was used as bandpass calibrator. Observations of the science target were interspersed with observations of the nearby quasar $\mathrm{J} 1625-2527\left(\approx 0.5 \mathrm{Jy} ; 1.8^{\circ}\right)$ that was used as phase calibrator. During the reduction, data of bad quality were flagged, partly automatically by the CASA tasks and partly through manual inspection. A phase-only self-calibration was performed on the continuum datasets and applied to the full data cubes before combining the $12 \mathrm{~m}$ array and ACA datasets and performing the final imaging. The resulting spectral line data cubes have a noise rms for the combined datasets of about 7-10 mJy beam ${ }^{-1}$ channel $^{-1}$, which translates into a uniform sensitivity of approximately 4-5 mJy beam ${ }^{-1} \mathrm{~km} \mathrm{~s}^{-1}$ with beam sizes ranging from $\approx 0.4-0.7^{\prime \prime}$ depending on the exact configuration at the date of observation (Table 1). For ease of comparison across the different spectral windows and extraction of spectra, a combined dataset with a circular restoring beam of $0.5^{\prime \prime}$ was produced as well.

\footnotetext{
2 The data are available through the ALMA archive at https:// almascience.eso.org/aq
} 
Table 1. Log of Band 7 observations.

\begin{tabular}{ccccll}
\hline \hline \multirow{2}{*}{ Setting } & \multirow{2}{*}{ Frequency [GHz] } & \multicolumn{2}{c}{ Observing date } & Number of antennas ${ }^{a}$ & Beam $^{b}$ \\
& & 12 m array & ACA & & \\
\hline a & $329.150-331.025$ & 2015-May-16 & 2014-Jun-08 & $41 / 10$ & $0.49^{\prime \prime} \times 0.37^{\prime \prime}\left(+77^{\circ}\right)$ \\
b & $331.025-332.900$ & $2015-M a y-17$ & 2014-Jun-14 & $36 / 9$ & $0.49^{\prime \prime} \times 0.40^{\prime \prime}\left(+87^{\circ}\right)$ \\
c & $332.900-334.775$ & 2015-May-17 & 2014-Jun-17 & $36 / 8$ & $0.55^{\prime \prime} \times 0.41^{\prime \prime}\left(-87^{\circ}\right)$ \\
d & $334.775-336.650$ & $2015-M a y-17$ & 2014-Jun-08 & $36 / 10$ & $0.43^{\prime \prime} \times 0.39^{\prime \prime}\left(+72^{\circ}\right)$ \\
e & $336.650-338.525$ & 2015-May-17 & 2015-Apr-04 & $36 / 9$ & $0.45^{\prime \prime} \times 0.39^{\prime \prime}\left(+79^{\circ}\right)$ \\
f & $338.525-340.400$ & 2015-May-21 & 2014-Jun-30 & $36 / 10$ & $0.44^{\prime \prime} \times 0.40^{\prime \prime}\left(+37^{\circ}\right)$ \\
g & $340.400-342.275$ & 2015-May-21 & 2014-Jun-14 & $36 / 9$ & $0.42^{\prime \prime} \times 0.38^{\prime \prime}\left(+56^{\circ}\right)$ \\
h & $342.275-344.150$ & 2015-May-21 & 2014-Jun-11 & $36 / 10$ & $0.43^{\prime \prime} \times 0.37^{\prime \prime}\left(+56^{\circ}\right)$ \\
i & $344.150-346.025$ & 2015-Apr.-05 & 2014-Jun-29 & $39 / 11$ & $0.87^{\prime \prime} \times 0.60^{\prime \prime}\left(+86^{\circ}\right)$ \\
j & $346.025-347.900$ & 2015-Apr.-05 & 2014-Jun-12 & $40 / 9$ & $0.81^{\prime \prime} \times 0.57^{\prime \prime}\left(+82^{\circ}\right)$ \\
k & $347.900-349.775$ & 2015-Apr.-04 & 2014-Jun-04 & $37 / 10$ & $0.82^{\prime \prime} \times 0.57^{\prime \prime}\left(+85^{\circ}\right)$ \\
l & $349.775-351.650$ & 2015-May-17 & 2014-Jun-10 & $36 / 8$ & $0.65^{\prime \prime} \times 0.39^{\prime \prime}\left(-81^{\circ}\right)$ \\
m & $351.650-353.525$ & 2015-Apr.-03 & 2014-Jun-10 & $38 / 8$ & $0.79^{\prime \prime} \times 0.58^{\prime \prime}\left(+79^{\circ}\right)$ \\
n & $353.525-355.400$ & 2015-May-19 & 2014-Dec-29 & $37 / 11$ & $0.44^{\prime \prime} \times 0.34^{\prime \prime}\left(-82^{\circ}\right)$ \\
o & $355.400-357.275$ & 2015-May-20 & 2014-Jun-17 & $37 / 8$ & $0.40^{\prime \prime} \times 0.37^{\prime \prime}\left(+45^{\circ}\right)$ \\
p & $357.275-359.150$ & 2015-May-20 & 2014-Jun-16 & $37 / 10$ & $0.42^{\prime \prime} \times 0.37^{\prime \prime}\left(+53^{\circ}\right)$ \\
q & $359.150-361.025$ & 2015-May-20 & 2014-Jun-14 & $37 / 9$ & $0.44^{\prime \prime} \times 0.38^{\prime \prime}\left(+60^{\circ}\right)$ \\
r & $361.025-362.900$ & 2015-May-21 & 2014-Jun-08 & $36 / 10$ & $0.43^{\prime \prime} \times 0.38^{\prime \prime}\left(+75^{\circ}\right)$ \\
\hline
\end{tabular}

Notes. ${ }^{(a)}$ Number of antennas available for the main array and ACA, respectively. ${ }^{(b)}$ Synthesized beam size and position angle for the $12 \mathrm{~m}$ and ACA datasets combined.

\subsubsection{Continuum subtraction}

For analysis and, in particular, the imaging of individual lines it is desirable to have continuum subtracted products. The standard procedure is to define one or more spectral regions with "line-free" channels to be used for continuum estimates and subtraction either in the $(u, v)$ - or image-plane. In this case, however, because of the high sensitivity (and dynamic range) the resulting image cubes are strongly line confused in large parts of the spectral ranges even toward IRAS 16293B with the narrowest lines. Also, some velocity gradients are seen, which makes a general definition of line-free regions impossible. Finally, fitting each spatial pixel by hand is not practical because of the large number of individual spectra (each datacube representing more than 10000 spectra and spectral coverage of more than 140000 independent channels).

To circumvent these issues and treat the data homogeneously, the continuum subtraction is done in a statistical/iterative manner: for each pixel in a datacube the density distribution of fluxvalues is created (Fig. 1). For a pixel with only a contribution from continuum emission (or noise) but no line emission, this distribution will represent a symmetric Gaussian centered at the continuum level with a width corresponding to the channelby-channel rms noise, $\sigma$. For pixels with both continuum and line emission this Gaussian will be modified by an exponential tail toward higher values with a number of datapoints at lower flux values in the case of absorption (in particular, toward IRAS 16293B). In either of these cases, however, the continuum level can still be recognized as the leading edge in the flux distribution (Fig. 1) with the overall distribution appearing as a log-normal or skewed Gaussian distribution.

The continuum is then determined in two automated steps: first, the peak of the distribution is determined over the entire range of the flux values with a simple symmetric Gaussian fit resulting in an estimate of the centroid $F$ and its width $\Delta F$. The width is small in case of pixels with little or no line emission, but for line contaminated regions the distribution becomes broader and the exact location of the peak more uncertain. Subsequently, a skewed Gaussian is fitted to the part of the distribution within $F \pm 3 \Delta F$ and the new centroid (now not necessarily symmetric) is recorded as the continuum level that can then be used for continuum subtraction for that particular pixel. This method does not fit the low flux tail corresponding to absorption lines, but generally these channels do not contribute significantly to the overall distribution. Experiments with the observed cubes and synthetic models suggest that the continuum level can be estimated in this manner to be accurate to within $2 \sigma$.

Figure 2 compares the continuum peak flux toward IRAS 16293B for each of the 18 spectral setups as a function of frequency. As expected a slight slope is seen with flux increasing as function of frequency consistent with a power-law $F_{v} \propto v^{\alpha}$ with $\alpha \approx 2$ as expected for optically thick dust continuum emission. This plot also provides a direct estimate of the calibration accuracy: the dark and light shaded areas correspond respectively to $\pm 5 \%$ and $\pm 10 \%$ around the continuum powerlaw. Thirteen out of the eighteen measurements (or 72\%) are within $\pm 5 \%$ while all of the measurements lie within the $\pm 10 \%$ ranges. Assuming that the scatter is entirely due to errors in the calibration, this suggests that the (flux) calibration accuracy is better than 5\% for the ALMA observations.

\subsection{Band 3 and Band 6 data: observations of specific spectral windows at 100 and $230 \mathrm{GHz}$}

In addition to the unbiased survey in Band 7, three spectral setups were observed in Band 6 and Band 3 between May and July 2014 targeting the same position. These setups were optimized to observe the emission from a range of glycolaldehyde lines previously measured in the laboratory as an extension of the detection of that molecule (Jørgensen et al. 2012) and to map species in the chemical network related to glycolaldehyde formation. Table 2 lists windows targeted in these setups and the logs of observations. For these settings only the $12 \mathrm{~m}$ array was utilized, and any more extended emission will thus be resolved 

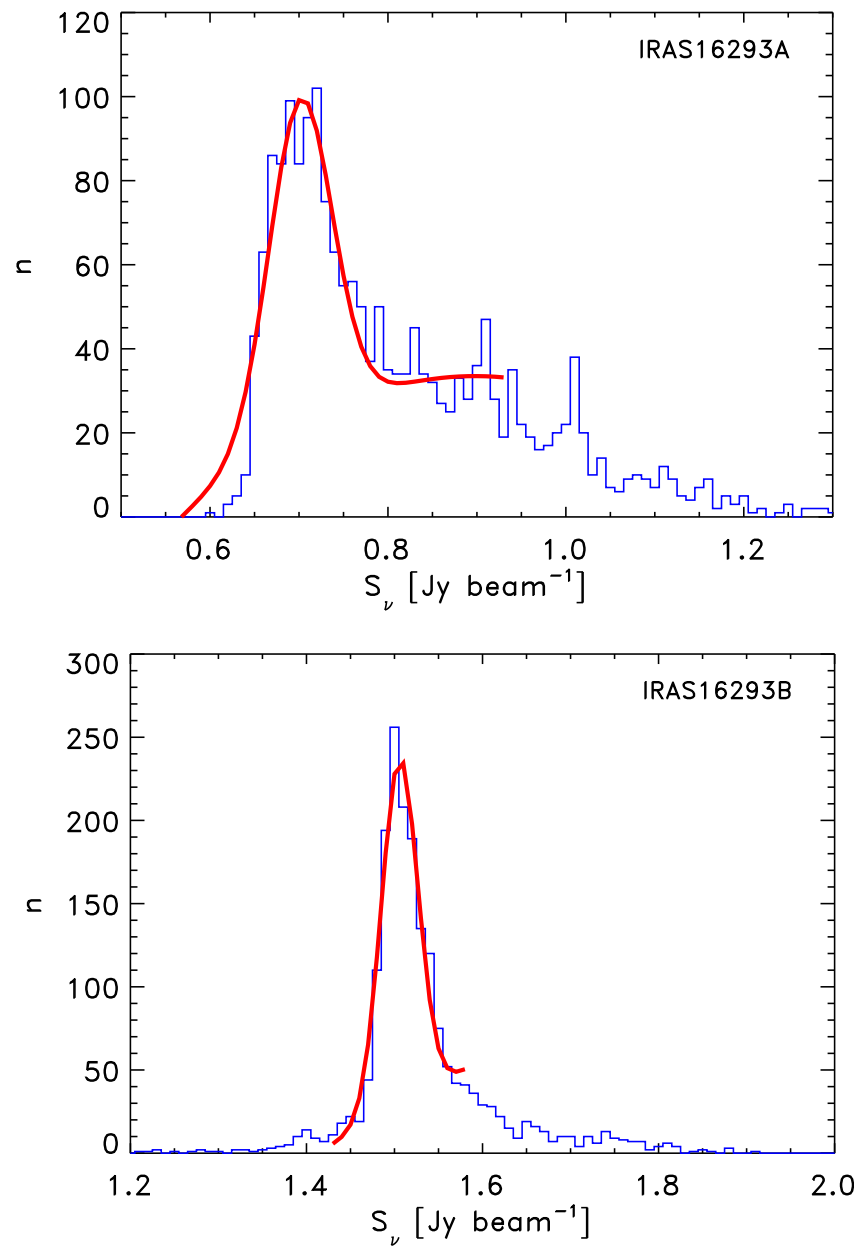

Fig. 1. Methodology for continuum subtraction illustrated with data from two representative pixels toward IRAS 16293A (upper) and IRAS 16293B (lower): shown are the flux distributions for the two pixels (histogram) with the resulting fit overlaid (red line).

out. The calibration otherwise proceeded in the same manner as for the Band 7 data.

\section{Results}

\subsection{Continuum emission}

Much can be learned about the structure of the IRAS 16293-2422 system by straightforward inspection of the dust continuum maps. Figure 3 shows a three-color composite of the continuum toward the system in the $3.0 \mathrm{~mm}$, $1.3 \mathrm{~mm}$, and $0.87 \mathrm{~mm}$ bands, while Fig. 4 shows the continuum at the three different wavelengths separately at the angular resolution of each individual dataset. The extended emission connecting the two sources, also noted in the science verification data (Pineda et al. 2012), is clearly seen. It shows a characteristic bend toward the north of IRAS 16293A/east of IRAS 16293B. East of IRAS 16293B two separate stream-lines pointing away from the source are seen. Toward IRAS 16293A additional extended continuum emission is observed toward the southwest. This extension coincides with the $\mathrm{N}_{2} \mathrm{D}^{+}$emission picked up in SMA observations (Jørgensen et al. 2011) and likely reflects cold material with a high column density.

Another very striking feature of the maps is the clear differences in the morphologies and colors of the emission toward the two protostars. IRAS 16293A appears clearly elongated in

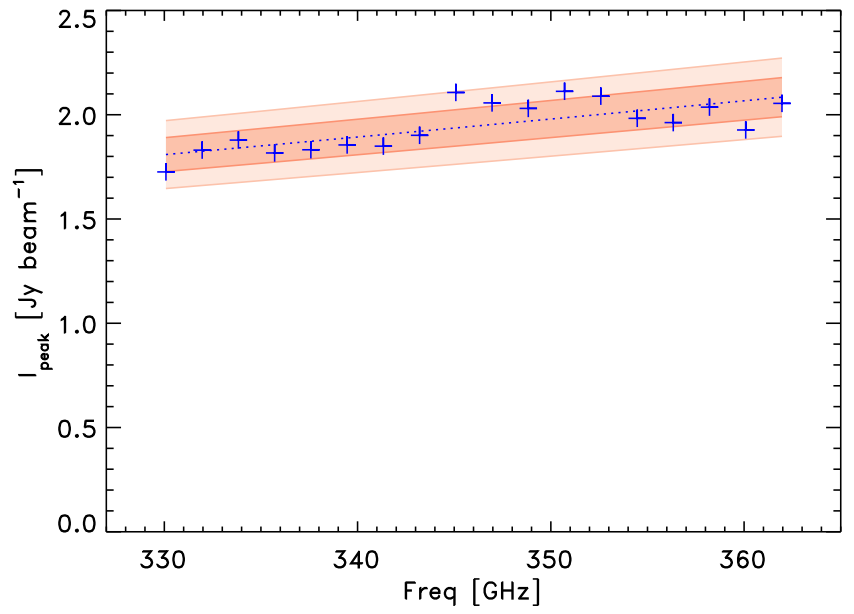

Fig. 2. Continuum peak flux (in a $0.5^{\prime \prime}$ beam toward IRAS $16293 \mathrm{~B}$ for the 18 different spectral setups (plus signs) as a function of frequency. A slight increase in flux as a function of frequency is seen that can be approximated by a power-law $F_{v} \propto v^{2}$. The shaded areas correspond to this dependency $\pm 5 \%$ (darker color) and $\pm 10 \%$ (lighter color). About $70 \%$ of the measurements are within the $\pm 5 \%$ region with all of the measurements within the $\pm 10 \%$ area.

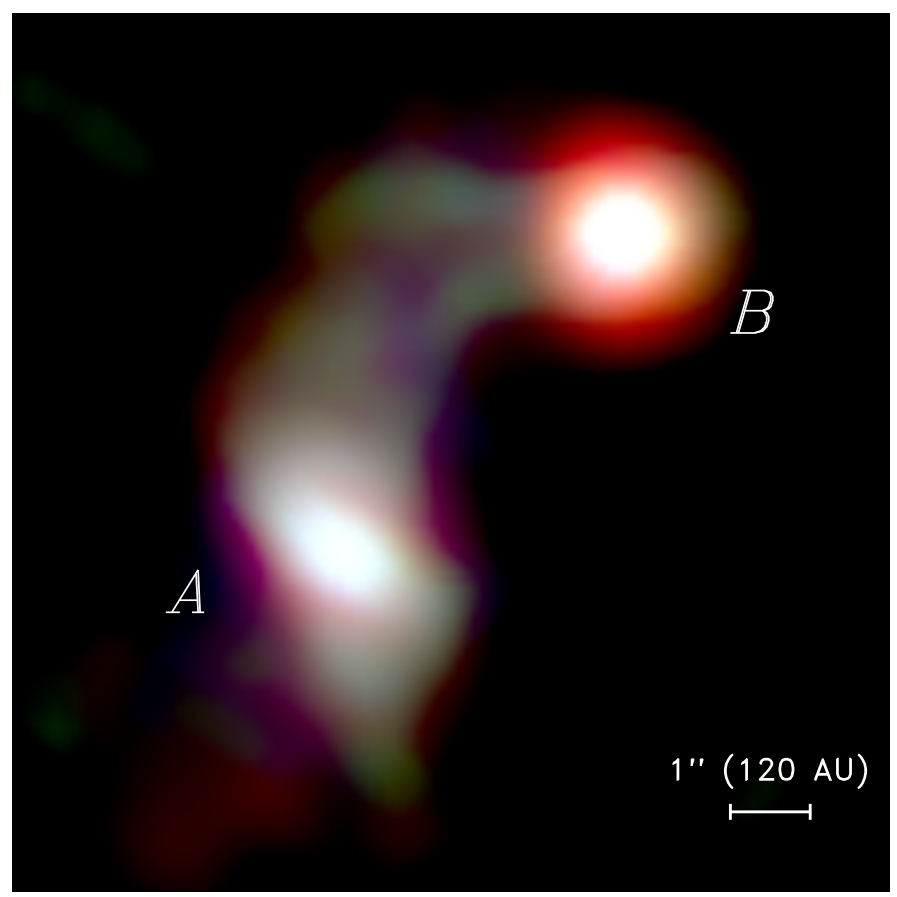

Fig. 3. Three-color image showing the continuum at $3.0 \mathrm{~mm}, 1.3 \mathrm{~mm}$, and $0.87 \mathrm{~mm}$ (ALMA Bands 3, 6, and 7) in red, green, and blue, respectively. Before the combination the $1.3 \mathrm{~mm}$ and $0.87 \mathrm{~mm}$ images were smoothed to match the resolution of the $3.0 \mathrm{~mm}$ data.

the northeast/southwest direction (with an aspect ratio of 1.9). Chandler et al. (2005) used continuum maps at $305 \mathrm{GHz}$ from the SMA to study the system: in super-resolution images (images for which higher weight was given to the longer baselines and subsequently restored with a beam slightly smaller than the usual synthesized beams), they noted a similar extension, but also found that the source broke up into two separate components named "Aa" and "Ab". These separate components are not seen in our images even though the beam in the ALMA observations (with regular weighting) is comparable to those super-resolution images. Our images are therefore more in 
Table 2. Log of ALMA Band 3 and Band 6 observations.

\begin{tabular}{llllll}
\hline \hline & Window & Frequency [GHz] & Date & Number of antennas & Beam $^{a}$ \\
\hline B3 & 0 & $92.78-93.02$ & 2014-Jul.-03 & 31 & $1.13^{\prime \prime} \times 0.93^{\prime \prime}\left(+15^{\circ}\right)$ \\
& 1 & $89.45-89.74$ & & & \\
& 2 & $102.48-102.73$ & & & \\
& 3 & $103.18-103.42$ & & 35 & $0.48^{\prime \prime} \times 0.43^{\prime \prime}\left(+64^{\circ}\right)$ \\
\hline B6-1 & 0 & $239.40-239.86$ & 2014-Jun.-14 & & \\
& 1 & $240.15-240.61$ & & & $0.62^{\prime \prime} \times 0.48^{\prime \prime}\left(+77^{\circ}\right)$ \\
& 2 & $224.75-225.21$ & & \\
\hline B6-2 & 0 & $221.76-222.22$ & & & \\
& 1 & $247.32-247.79$ & $2014-$ May-22 & 35 & \\
& $250.28-250.75$ & & & & \\
& 3 & $231.03-231.51$ & & & \\
\hline
\end{tabular}

Notes. ${ }^{(a)}$ Synthesized beam size and position angle for the dataset.

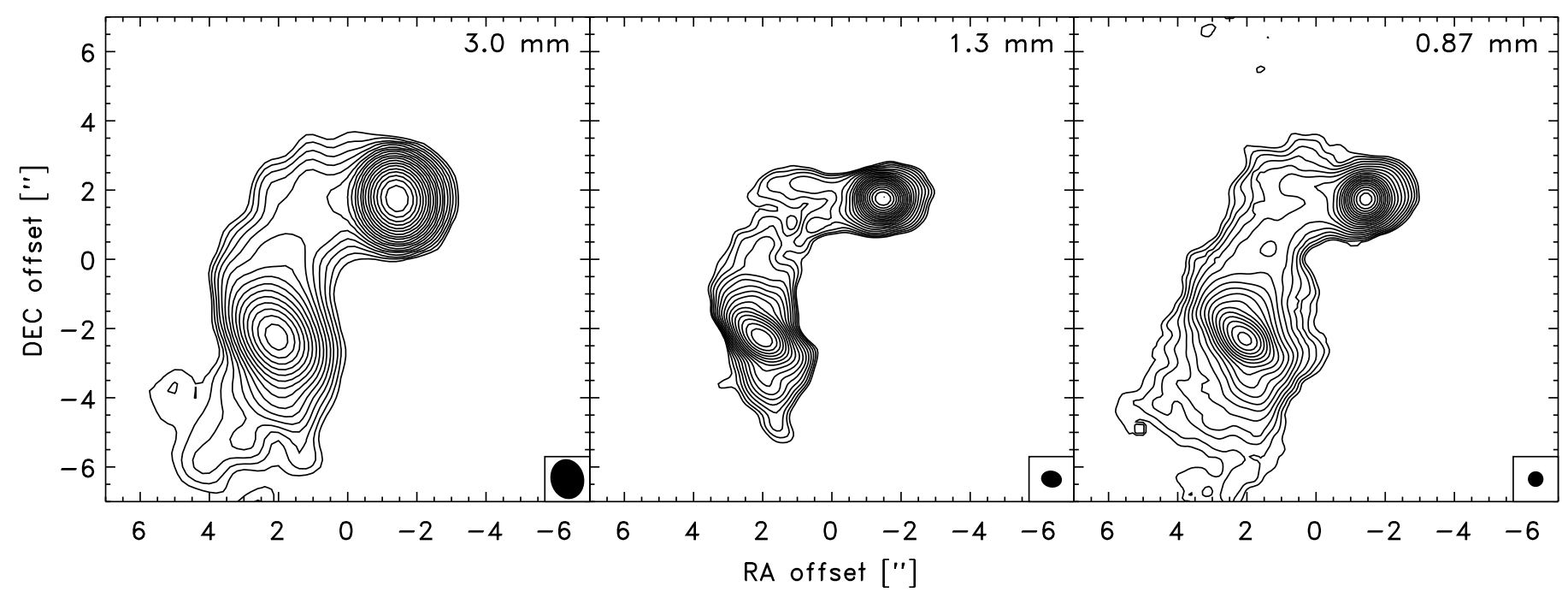

Fig. 4. Continuum images at 3.0, 1.3, and $0.87 \mathrm{~mm}$ (left, middle, and right) at the angular resolution of each dataaset. The $0.87 \mathrm{~mm}$ images include both $12 \mathrm{~m}$ array and ACA data, while the 3.0 and $1.3 \mathrm{~mm}$ images only contain data from the $12 \mathrm{~mm}$ array. The contour levels are given as 20 logarithmically divided levels between $0.5 \%$ and $100 \%$ of the peak flux at the given wavelength. The RA and Dec offsets are relative to the phase center for the observations.

support of the interpretation that the submillimeter continuum emission toward IRAS 16293A represents a more edge-on disk system, also supported by the velocity gradient seen in this direction (Pineda et al. 2012; Girart et al. 2014).

In stark contrast, the emission toward IRAS 16293B is very circular with an elliptical aspect ratio $<1.1$. Its colors are also much redder than both the extended emission and that toward IRAS 16293A. This is consistent with the suggestion that the emission toward IRAS 16293B is optically thick at the $50 \mathrm{AU}$ scales imaged here and at higher frequencies (e.g., Zapata et al. 2013). The fact that it appears so optically thick at even longer wavelengths tightens the constraints on the minimum amount of dust toward IRAS 16293B: assuming typical dust opacities from Ossenkopf \& Henning (1994) with $\kappa_{v}=0.0182 \mathrm{~cm}^{2} \mathrm{~g}^{-1}$ at $850 \mu \mathrm{m}$ an optical depth $\tau_{v}>1$ implies that the column density toward the continuum peak is

$N\left(\mathrm{H}_{2}\right)=\frac{\tau_{v}}{\mu_{\mathrm{H}_{2}} m_{\mathrm{H} \kappa_{v}}}>1.2 \times 10^{25} \mathrm{~cm}^{-2}$

and consequently for a distribution over a $50 \mathrm{AU}$ (diameter) projected circular region that the mass is $\gtrsim 0.01 M_{\odot}$. While this may not appear to be a significant amount, it is in fact 1-2 orders of magnitude above the mass on similar scales from larger scale envelope models (e.g., Schöier et al. 2002) for IRAS 16293-2422 extrapolated to these scales. Likewise, if it is assumed that the distribution of the material along the line of sight is comparable to the projected extent on the sky the lower limit to the column density translates into a density $\gtrsim 3 \times 10^{10} \mathrm{~cm}^{-3}$; if this dust is indeed located in a face-on disk-like structure such as implied by the circular distribution and narrow line widths toward the source (e.g., Jørgensen et al. 2011), the density is expected to be even higher than this lower limit.

\subsection{Line emission}

The incredible line-richness of IRAS 16293-2422 makes it a natural template source for astrochemical studies. Figure 5 shows the full spectrum from the Band 7 data toward a position offset by $0.25^{\prime \prime}$ (a half beam) from IRAS 16293B that is used for the analysis in this paper, while the figures in Appendix B show the spectra from the Band 3 and 6 data. Figure 6 compares the observed spectra in the $338-339 \mathrm{GHz}$ spectral range (including 


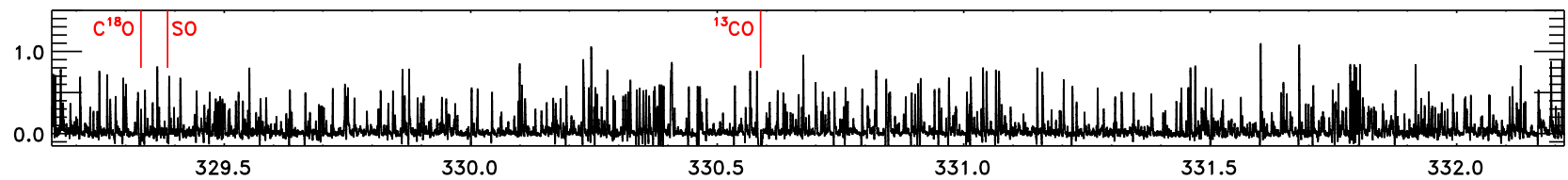
1.0 10. 19.

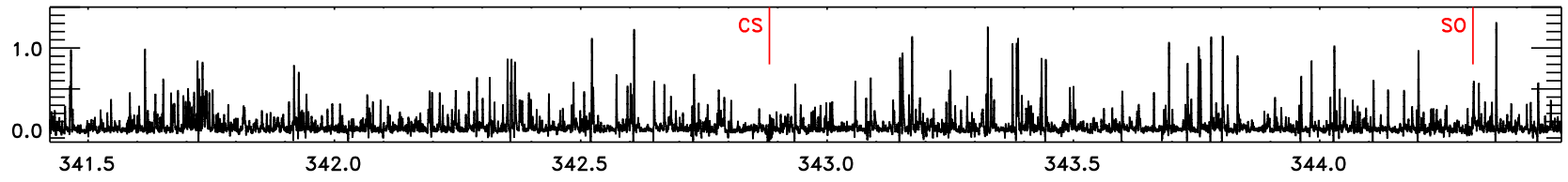

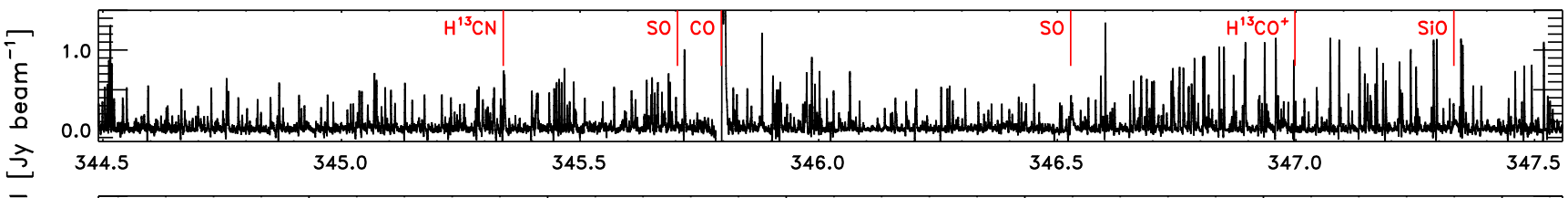

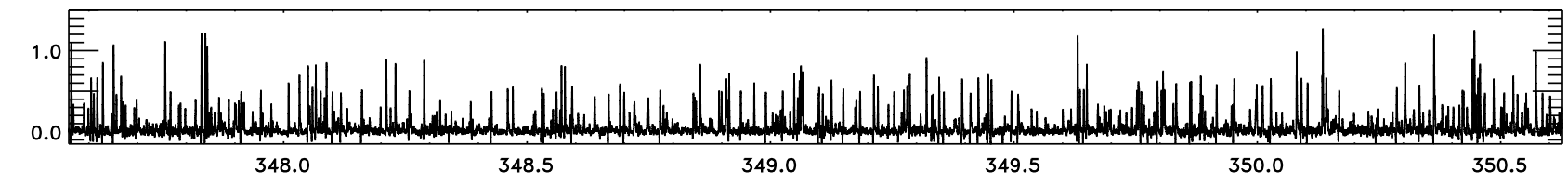
10 10

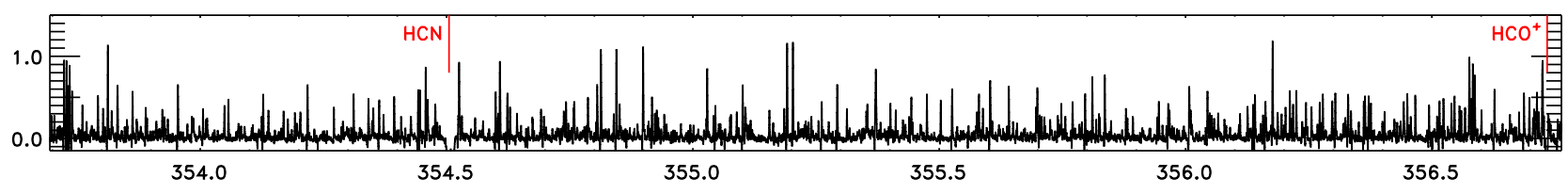
10 10

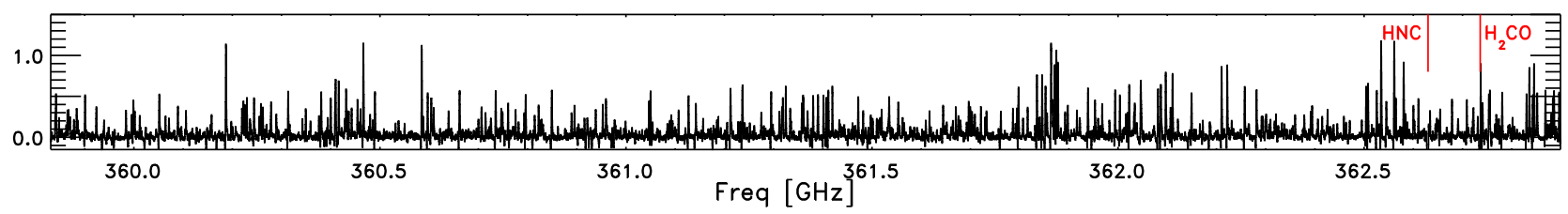

Fig. 5. Spectrum in a $0.5^{\prime \prime}$ beam toward a position offset by half a beam from the continuum peak of IRAS 16293B. Frequencies of prominent lines of a few key species are marked by vertical lines. 
the main $\mathrm{CH}_{3} \mathrm{OH} 7_{k}-6_{k}$ branch) from JCMT observations taken as part of the larger single-dish TIMASSS survey (Caux et al. 2011) to spectra toward the two continuum peaks in the ACAonly and full ALMA datasets. Also shown are spectra toward positions separated by a half and a full beam from IRAS 16293B, respectively.

The difference in line widths between IRAS 16293A and IRAS 16293B noted previously (e.g., Bottinelli et al. 2004b; Jørgensen et al. 2011) is clearly seen in Fig. 6 with the lines toward IRAS 16293A about a factor of 5 broader than the narrow $\approx 1 \mathrm{~km} \mathrm{~s}^{-1}$ (FWHM) lines toward IRAS 16293B. This difference is consistent with the interpretation above that IRAS 16293A is an edge-on system and IRAS 16293B face-on. The very narrow line widths for IRAS 16293B make it an ideal source for line identifications compared to, e.g., the Galactic Center and typical high-mass star formation regions with widths of 5-10 $\mathrm{km} \mathrm{s}^{-1}$ Furthermore, the strong variations in the peak strengths of the different lines between all seven panels is a clear indication that the excitation conditions and/or chemistry change significantly over the studied scales.

The single-dish spectrum with its beam of approximately $14^{\prime \prime}$ naturally encompasses both IRAS 16293A and IRAS 16293B: the line widths in this particular spectrum are generally closer to IRAS 16293A suggesting that the data are more strongly weighted toward this source. Again, this is consistent with the emission being more extended, and thus dominating in the single-dish beam, even though the peak strengths are not significantly different. Some of the brighter lines in the singledish spectrum are in fact stronger than in the ACA data - as is expected - but there are also counter-examples. This likely indicates that the single-dish observations were not targeted exactly between the two sources, and thus for slightly extended transitions some of the flux is not picked-up in full by the single-dish spectra. The point source rms noise level in the single-dish data is $0.15-0.3 \mathrm{Jy}_{\text {beam }}{ }^{-1} \mathrm{~km} \mathrm{~s}^{-1}$, whereas the ACA-only data is already about a factor of 5 more sensitive. The combined dataset is an additional order of magnitude more sensitive with a rms level of $5 \mathrm{mJy}$ beam $^{-1} \mathrm{~km} \mathrm{~s}^{-1}$. Furthermore the ALMA observation produced fully sampled maps with a higher angular resolution.

While comparing the ACA-only and combined ALMA data, a very striking feature is the emergence of a number of absorption lines. This is one of the key arguments for not considering the continuum peak position of IRAS 16293B for line identification and modeling. Instead we extract the spectra at positions half a beam and one beam southeast of the continuum peak (see Fig. 7 as well as higher angular resolution Band 9 data of Baryshev et al. 2015; their Fig. 13). As seen in Fig. 6 this shift means that most lines become pure emission lines: the peak of line emission is located at a position offset by half a beam from the continuum peak, but some of the brighter lines still show self-absorption there. At the position separated one beam from the continuum peak, very few absorption features remain, but the fluxes of lines that are not strongly optically thick is also weakened by a factor of $\approx 1.8$, likely reflecting the drop in column density moving away from the center: in the Band 6 data, the continuum flux drops by factors of $\approx 2.2$ and 4.7 by going from the peak position to the positions offset by a half and full beam, respectively, reflecting the drop in column density and, to a smaller degree, temperature. Which of the positions is preferable for the analysis depends on the species considered. It should be emphasized that for species with similar distributions, it is possible either to compare column densities derived at the same positions or to apply the correction factor above for statements about relative abundances.
Over the full $33.7 \mathrm{GHz}$ spectral range approximately 10000 lines are seen toward the position half a beam away from the continuum peak, i.e., on average 1 line per $3.4 \mathrm{MHz}$. Toward this position the average line flux per channel is $60 \mathrm{mJy}^{\text {beam }}{ }^{-1}$, whereas the continuum flux is $1.1 \mathrm{Jy} \mathrm{beam}^{-1}$, i.e., approximately a $5 \%$ contamination of the continuum by line emission. The line density is about a factor of 10 higher than was found by Caux et al. (2011) in the part of the TIMASSS single-dish survey covering the same frequencies. This improvement is in part due to the higher sensitivity of the ALMA, but also reflects the narrower lines toward IRAS 16293B in the spatially resolved ALMA data making it easier to separate individual features. Compared to single-dish studies of IRAS 16293 (e.g., Blake et al. 1994; van Dishoeck et al. 1995; Caux et al. 2011) it is noteworthy that the lines from the most common species do not stand out prominently close to the continuum peaks, partly because of the high optical depth for the transitions of the most common isotopologues causing them to be in absorption against the continuum. This is, for example, seen for species such as $\mathrm{CO}, \mathrm{HCO}^{+}, \mathrm{CS}$, and $\mathrm{HCN}$. For some of their rarer isotopologues the emission lines are seen, but their line brightness at the scales of the interferometric data are not particularly high compared to the lines from the complex organics. This is in contrast to singledish observations that also pick up the more extended emission of the more common species and lower excited transitions.

While assigning all of the lines seen in Fig. 5 is beyond the scope of this initial paper, a statistical argument can be made for the nature of these 10000 lines. Based on previous estimates of the column densities of complex organic molecules toward IRAS 16293B we calculate synthetic spectra for the most common organics seen toward hot cores and hot corinos: formaldehyde, methanol, methyl cyanide, isocyanic acid, ethanol, acetaldehyde, methyl formate, dimethyl ether, and ketene. The model predicts that approximately $25 \%$ of the transitions in Fig. 5 are from the main isotopologues of these species. In addition, some regular non-organic species have lines in the frequency range, but the bulk of the remaining species can likely be attributed to isotopologues of the complex organics $\left({ }^{13} \mathrm{C},{ }^{18} \mathrm{O}\right.$, ${ }^{17} \mathrm{O},{ }^{15} \mathrm{~N}, \mathrm{D}$, and all possible combinations) appearing at the sensitivity of the ALMA observations (Coutens et al. 2016, and Sect. 5 of this paper) as well as the "next level" of complex organics, i.e., species with three carbon atoms (e.g., Lykke et al., in prep.).

\section{Analysis}

In this section we present an analysis of the ALMA data focusing, in particular, on the simple sugar-like molecule, glycolaldehyde, previously detected in the Science Verification data (Jørgensen et al. 2012) and related species. Section 5.1 analyzes the assumption of LTE at the densities representative for the scales traced by the ALMA data and presents an estimate of the column density for methanol useful as a future reference for abundance measurements. Section 5.2 analyzes the emission from glycolaldehyde and the related species, ethylene glycol (the reduced alcohol of glycolaldehyde) and acetic acid (the third isomer in the glycolaldehyde/methyl formate group), while Sect. 5.3 presents detections of the ${ }^{13} \mathrm{C}$ and deuterated isotopologues of glycolaldehyde.

\subsection{Excitation analysis at observed scales}

The dust continuum emission from the material on the scales probed by ALMA provides important constraints on the physical 
J. K. Jørgensen et al.: The ALMA Protostellar Interferometric Line Survey (PILS)
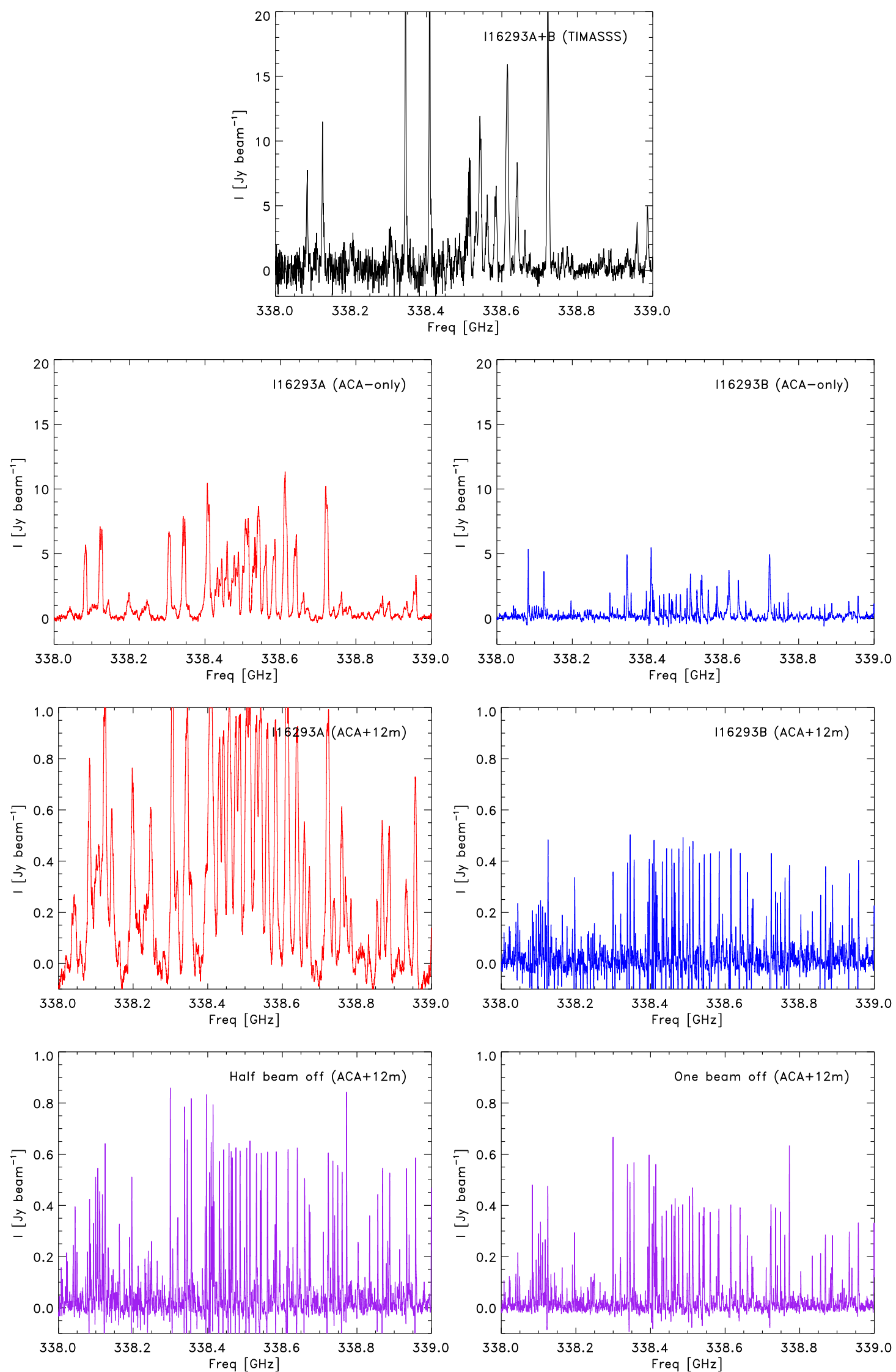

Fig. 6. Comparison between the spectra in the $338 \mathrm{GHz}$ window around the prominent $\mathrm{CH}_{3} \mathrm{OH}$ branch from the single-dish observations from the TIMASSS survey (Caux et al. 2011) (top row), the ACA-only data toward IRAS 16293A and IRAS 16293B (second row; left and right, respectively), the full data toward IRAS 16293A and IRAS 16293B (third row; left and right, respectively), and positions offset by a half and full beam southwest of the IRAS 16293B continuum position (bottom row). The scale on the $Y$-axis in the bottom two rows is different from the top rows. 

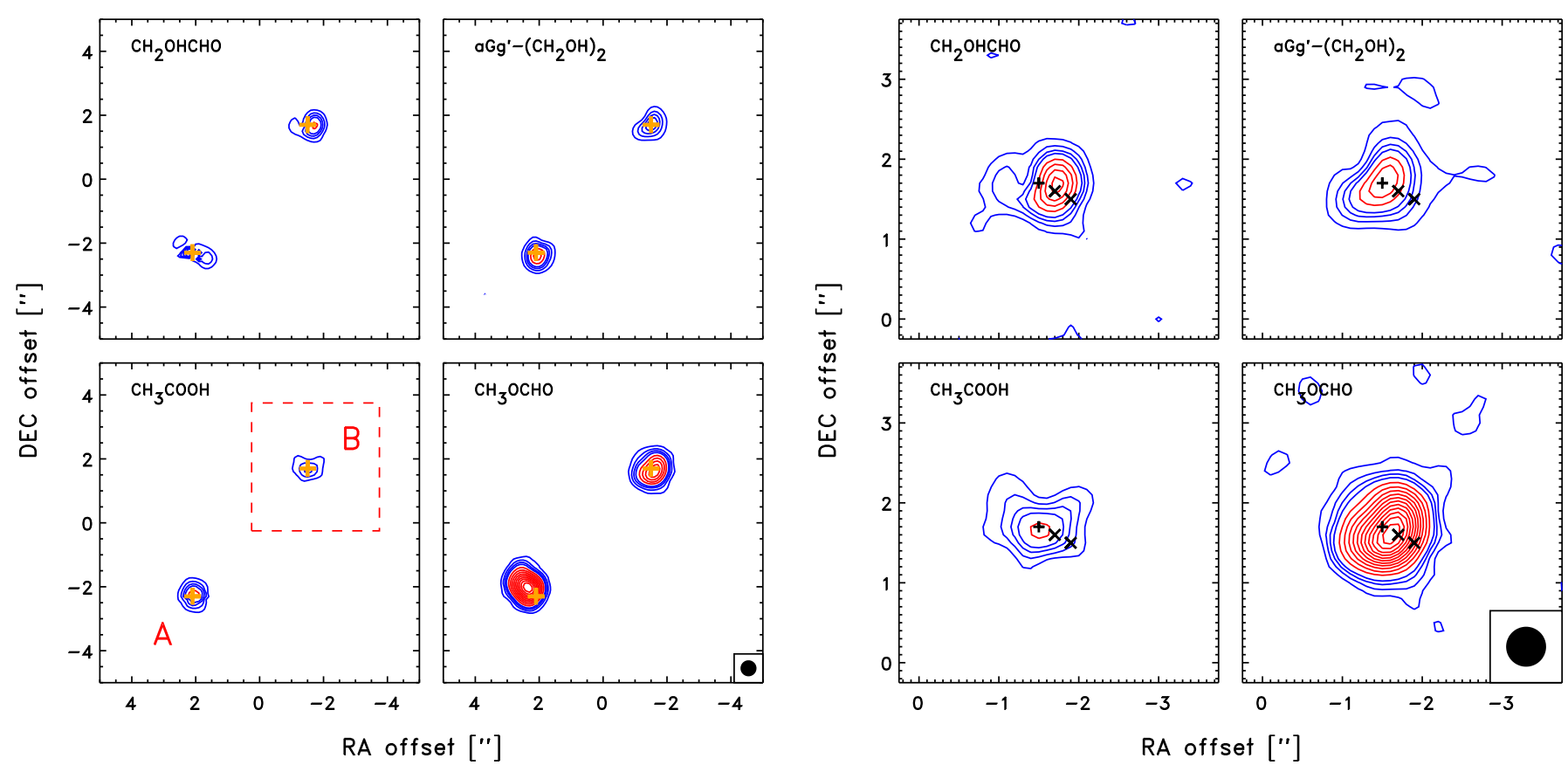

Fig. 7. Maps of representative transitions of glycolaldehyde and related species. The four panels to the left show the entire IRAS $16293-2422$ system, while the four panels to the right zoom-in on IRAS 16293B (indicated as the red dashed square in the lower left panel). The mapped species are glycolaldehyde $\left(362.406 \mathrm{GHz}, E_{\text {up }}=266 \mathrm{~K}\right.$; upper left $)$, ethylene glycol $\left(348.550 \mathrm{GHz}, E_{\text {up }}=329 \mathrm{~K} ;\right.$ upper right $)$, acetic acid $(352.872 \mathrm{GHz}$, $E_{\text {up }}=275 \mathrm{~K}$; lower left $)$, and methyl formate $\left(360.467 \mathrm{GHz}, E_{\mathrm{up}}=324 \mathrm{~K}\right.$; lower right $)$. In each map the contours represent the integrated emission over $\pm 0.5 \mathrm{~km} \mathrm{~s}^{-1}$ in steps of $15 \mathrm{mJy} \mathrm{km} \mathrm{s}^{-1}$ (approximately $3 \sigma$ ). In each panel the plus sign marks the location of the continuum sources associated with IRAS 16293A and IRAS 16293B (labeled A and B in the lower left panel), while the crosses in the right panels represent the positions offset by half a beam and a full beam from IRAS 16293B. These are the positions used for line identification/modeling.

structure - and thus the molecular excitation. Ideally, the full non-LTE radiative transfer problem needs to be solved to use the line observations, for example, for constraints on column densities or abundances. However, for most of the complex organic molecules dominating the spectra, no collisional rate coefficients are available and such calculations are therefore not possible. However, the high densities implied from the optical thickness of the dust continuum emission (Sect. 4.1) means the calculations under the assumption of LTE are likely to be sufficient.

To test the validity of the LTE assumption, we use the escape probability code Radex (van der Tak et al. 2007) to solve the radiative transfer problem for the largest complex molecule methanol $\left(\mathrm{CH}_{3} \mathrm{OH}\right)$, for which collision rates are available from the Leiden Atomic and Molecular Database (LAMDA; Schöier et al. 2005) based on calculations by Rabli \& Flower (2010). For a range of densities, $n\left(\mathrm{H}_{2}\right)$, and kinetic temperatures, $T_{\text {kin }}$, we calculate the excitation temperatures, $T_{\mathrm{ex}, i}$, for each of the $\mathrm{CH}_{3} \mathrm{OH}$ transitions (both A- and E-type) in the 329 to $363 \mathrm{GHz}$ range of the survey. We then estimate the root mean square deviation between each of these excitation temperatures and the kinetic temperature

$T_{\mathrm{rms}}=\sqrt{\frac{1}{N} \sum_{i}\left(T_{\mathrm{ex}, i}-T_{\mathrm{kin}}\right)^{2}}$,

where $N$ is the total number of transitions in the band: $N=82$ in the frequency range from 329 to $363 \mathrm{GHz}$. The value of $T_{\text {rms }}$ thereby provides a measure of whether LTE is a good approximation in general for the specific value of $n\left(\mathrm{H}_{2}\right)$ and $T_{\text {kin }}$ : if LTE is a good approximation for a given transition, then $T_{\text {ex }, i} \approx T_{\text {kin. }}$. A low $T_{\text {rms }}$ would therefore signify that most transitions are well reproduced in LTE.
Figure 8 shows the result of these excitation calculations for $\mathrm{CH}_{3} \mathrm{OH}$ for models with $T_{\text {kin }}$ of 50,100 , and $300 \mathrm{~K}$, respectively, with densities varying from $10^{6}-10^{12} \mathrm{~cm}^{-3}$. At low densities significant fluctuations due to transitions masing at specific densities are seen. Still, at those densities even the brighter transitions are subthermally excited, leading to the very large rms deviation between the individual excitation temperatures and the adopted value for $T_{\text {kin }}$. However, for each set of models $T_{\text {rms }} / T_{\text {kin }}$ clearly decreases at densities higher than $10^{9}-10^{10} \mathrm{~cm}^{-3}$. For densities higher than $3 \times 10^{10} \mathrm{~cm}^{-3}$, the lower limit according to the continuum calculations (Sect. 4.1), the deviations are less than $15 \%$ for these three kinetic temperatures, which is a confirmation that the excitation is close to being in LTE. In conclusion, with the lower limit to the density at the scales of the ALMA observations implied by the continuum optical depth, LTE is a good approximation for $\mathrm{CH}_{3} \mathrm{OH}$ and presumably for most other molecules.

Although it is often assumed that the dust continuum emission is optically thin at submillimeter wavelengths and thus a tracer of column density, this is not the case for IRAS 16293B and the column density quoted in Sect. 4.1 is at best a lower limit to the actual column density of material at these scales. Abundance estimates are therefore much better expressed relative to a chemically related molecule. To supply such a reference we fitted the observed lines from $\mathrm{CH}_{3}{ }^{18} \mathrm{OH}$ in the Band 7 data with synthetic spectra (Fig. C.1). As indicated in Fig. 5 and the figures in Appendix B, the Band 7 and 6 data are approaching the line confusion level at specific frequencies. For this reason it is not practical to identify individual lines and create rotation diagrams based on them. Instead, the approach taken for this analysis, and for other species in Bands 6 and 7, is to calculate synthetic spectra of the targeted molecules and directly compare them to the 


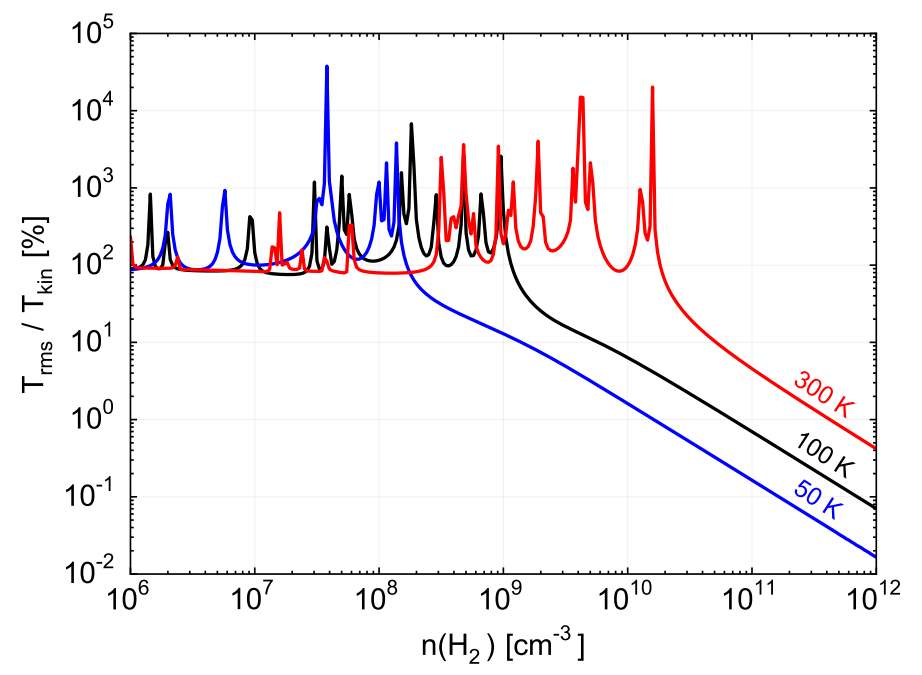

Fig. 8. Excitation of $\mathrm{CH}_{3} \mathrm{OH}$ transitions. The lines show the rms deviation $T_{\mathrm{rms}}$ for the excitation temperatures, $T_{\mathrm{ex}}$, relative to the kinetic temperature from calculations with the Radex escape probability code (van der Tak et al. 2007) for all $\mathrm{CH}_{3} \mathrm{OH}$ transition in the 329 to $363 \mathrm{GHz}$ band as a function of $\mathrm{H}_{2}$ density. The three different lines show results for kinetic temperatures of 50,100 , and $300 \mathrm{~K}$ in blue, black, and red, respectively.

extracted spectra. This model also contains a reference model for other identified species and thereby makes it possible to address whether a given line is a genuine identification. Following the arguments above, the synthetic spectra are computed under the assumption that the excitation obeys LTE. The parameters needed to produce a synthetic spectrum are then the column densities and excitation temperature of the particular molecule, the extent of the emission, and the line width and LSR velocity. We adopt a source size of $0.5^{\prime \prime}$, justified by the maps shown in Fig. 7; the maps of the emission from the complex organics in general show a centrally condensed component toward IRAS 16293B, which is marginally resolved with an extent of $0.6-0.9^{\prime \prime}$ consistent with a deconvolved extent of approximately $0.5^{\prime \prime}$ (see also Coutens et al. 2016; and Lykke et al., in prep.). It should be noted that since the main purpose is to establish a reference scale for comparison between relative abundances of different complex organics present in the same gas based on optically thin transitions, the exact value of the intrinsic source size is less critical. In this analysis we keep the line width and LSR velocity fixed, which appears to reproduce all lines well at the position half a beam from the continuum peak.

Since we aim to use $\mathrm{CH}_{3}{ }^{18} \mathrm{OH}$ lines to provide a reference column density for comparison to other species we adopt here an excitation temperature of $300 \mathrm{~K}$, which appears to be required from modeling of isotopologues of formamide $\left(\mathrm{NH}_{2} \mathrm{CHO}\right)$, isocyanic acid (HNCO), and glycolaldehyde and ethylene glycol (Jørgensen et al. 2012, and the following section). As seen in Fig. C.1, most of the transitions are in fact well modeled with this excitation temperature. Two counter-examples are the lowest excitation transitions ( $E_{\mathrm{up}}$ of 16 and $35 \mathrm{~K}$, respectively) for which colder material may contribute to the observed line intensities. Also, a few other examples of worse fits are seen where the $\mathrm{CH}_{3}^{18} \mathrm{OH}$ is blended with other species, e.g., the transition at $343.135 \mathrm{GHz}\left(E_{\text {up }}=204 \mathrm{~K}\right)$ that is located between two bright methyl formate transitions (at 343.134 and $343.136 \mathrm{GHz}$ ) or the $345.858 \mathrm{GHz}$ and $361.052 \mathrm{GHz}$ transitions $\left(E_{\text {up }}=326\right.$ and $338 \mathrm{~K}$ ) that overlap with the lines of ethylene glycol and formamide, respectively (the latter in fact strongly self-absorbed; see also Coutens et al. 2016). Still, besides these cases, it appears that there is no systematic over- or underestimate of either the lowest or highest excitation transitions, an indication that the adopted excitation temperature is representative of the bulk of the gas at this position. All of the lines are well-fit with one LSR velocity and width, which indicates that this single warm component dominates the emission at the scales of the ALMA beam and is relatively homogeneous in terms of its physical conditions.

With the excitation temperature of $300 \mathrm{~K}$, the derived column density for $\mathrm{CH}_{3}{ }^{18} \mathrm{OH}$ is $4 \times 10^{16} \mathrm{~cm}^{-2}$ within a $0.5^{\prime \prime}$ beam toward the position offset by half a beam from the continuum peak, corresponding to a column density of the main $\mathrm{CH}_{3} \mathrm{OH}$ isotopologue of $2 \times 10^{19} \mathrm{~cm}^{-2}$ with a standard interstellar medium (ISM) ${ }^{16} \mathrm{O} /{ }^{18} \mathrm{O}$ ratio of 560 (Wilson \& Rood 1994) or an upper limit to its abundance of $3 \times 10^{-6}$ with respect to $\mathrm{H}_{2}$ using the lower limit to the total column density from the continuum emission. Again, we emphasize that even as a limit the abundance with respect to $\mathrm{H}_{2}$ is highly uncertain and dependent on the exact relation between the physical components traced by the line and continuum emission. However, for molecules distributed similarly to $\mathrm{CH}_{3} \mathrm{OH}$ and thought to be chemically related, the abundance reference relative to $\mathrm{CH}_{3} \mathrm{OH}$ is useful.

\subsection{Glycolaldehyde, ethylene glycol, and acetic acid}

Glycolaldehyde $\left(\mathrm{CH}_{2} \mathrm{OHCHO}\right)$ is interesting from an astrobiological point of view as it is a simple sugar-like molecule and under Earth-like conditions is related to the formation of ribose. Since its first detection toward the Galactic Center (Hollis et al. 2000, 2001, 2004; Halfen et al. 2006; Requena-Torres et al. 2008) it has been detected in a number of other places in the ISM, both associated with the formation of stars of high (Beltrán et al. 2009; Calcutt et al. 2014), intermediate (Fuente et al. 2014), and low mass (Jørgensen et al. 2012; Coutens et al. 2015; Taquet et al. 2015). Recently, glycolaldehyde has also been detected in comet C/2014 Q2 (Lovejoy) (Biver et al. 2015). Ethylene glycol is closely related to glycolaldehyde being its reduced alcohol version. It has been seen toward most of the regions where glycolaldehyde is detected and toward a few more high-mass star-forming regions (Lykke et al. 2015), the Orion-KL nebula (Brouillet et al. 2015) and toward a few comets where it appears to be more abundant than glycolaldehyde (e.g., Crovisier et al. 2004; Biver et al. 2014). Two other species worth mentioning are the two isomers of glycolaldehyde, methyl formate $\left(\mathrm{CH}_{3} \mathrm{OCHO}\right)$ and acetic acid $\left(\mathrm{CH}_{3} \mathrm{COOH}\right)$. The former is relatively common in high-mass star-forming regions (e.g., Brown et al. 1975; Ellder et al. 1980; Blake et al. 1987; MacDonald et al. 1996; Gibb et al. 2000; Bisschop et al. 2007) and has also been detected toward low-mass protostars (e.g., Cazaux et al. 2003; Bottinelli et al. 2004a; Jørgensen et al. 2005a; Sakai et al. 2006) and even prestellar cores (e.g., Öberg et al. 2010; Bacmann et al. 2012), while acetic acid is less abundant (Mehringer et al. 1997; Remijan et al. 2002) and tentatively detected toward low-mass protostars (Cazaux et al. 2003; Shiao et al. 2010). Studies of the relative abundances of these species may provide interesting insights into their formation and possibly also provide the link between the physical conditions in star-forming regions and the solar system. For example, significant differences are seen between the relative abundances of ethylene glycol and 
glycolaldehyde between different regions of high- and low-mass star-forming regions and comets (e.g., Lykke et al. 2015) with low glycolaldehyde abundances relative to ethylene glycol seen in some star-forming regions and comets but the Galactic Center shows comparable abundances of the two species (Hollis et al. 2002; Belloche et al. 2013).

\subsubsection{Spectroscopic data and vibrational corrections}

The spectroscopic data used in the analysis here have different origins: the data for glycolaldehyde are based on laboratory measurements by Butler et al. (2001), Widicus Weaver et al. (2005) and Carroll et al. (2010) including the $v=0-3$ forms and are provided by the JPL catalogue (Pickett et al. 1998). The JPL catalogue also supplies data for methyl formate based on measurements by Ilyushin et al. (2009). The data for acetic acid were taken from the Spectral Line Atlas of Interstellar Molecules (SLAIM), which is available through the Splatalogue ${ }^{3}$ interface (F. J. Lovas, priv. comm.; Remijan et al. 2007), with the partition function given by Mehringer et al. (1997). Ethylene glycol has a more complex structure than the other species: it is a triple rotator with coupled rotation possible around the C-C and two $\mathrm{C}-\mathrm{O}$ bonds. This means that ethylene glycol can be characterized by a total of ten different conformers distributed over the two main groups (gauche and anti), characterized by the arrangements of the two $\mathrm{OH}$ groups (the gauche forms more energetically favorable). Of these, the lowest state $a G g^{\prime}$ conformer is the one that has been detected in star-forming regions. The next lowest $g G g^{\prime}$ conformer lies about $290 \mathrm{~K}$ higher than the $a G g^{\prime}$ conformer (Müller \& Christen 2004); it has been searched for, but not detected, in the Orion hot core with an upper limit of 0.2 times the column density of the $a G g^{\prime}$ conformer (Brouillet et al. 2015). In the ALMA Science Verification data, a single line of this conformer was tentatively detected (Jørgensen et al. 2012), but no lines of the main conformer were covered in the observed spectral range. The spectroscopy of the two conformers is provided by the Cologne Database for Molecular Spectroscopy (CDMS; Müller et al. 2001, 2005) based on measurements by Christen \& Müller (2003) and Müller \& Christen (2004) for the $a G g^{\prime}$ and $g G g^{\prime}$ conformers, respectively.

The high temperatures on the scales of the ALMA observations ( $300 \mathrm{~K}$ Jørgensen et al. 2012, and below) complicates the direct use of the tabulated spectroscopic data and partition functions. In the case of an organic molecule with several lowlying vibrational modes, such as glycolaldehyde, the high temperatures mean that several vibrational states will be populated in addition to the vibrational ground state. The infrared spectrum of gas-phase glycolaldehyde was measured quite recently (Johnson et al. 2013). There are three fundamental modes below $520 \mathrm{~K}$ vibrational energy. These fundamentals have been considered in the calculation of the partition function for the JPL catalogue entry. They increase the partition function by a factor of $\sim 1.9$ at $300 \mathrm{~K}$. Partition function values in the CDMS entries refer to the ground state only. Accounting for the associated overtone and combination states in the harmonic oscillator approximation and lowering the $v_{17}$ mode because of its pronounced anharmonicity yields a vibrational factor of 2.59 , which increases to 2.80 upon consideration of the next three modes below $1250 \mathrm{~K}$. All other modes are above $1550 \mathrm{~K}$ and contribute combined less than $2 \%$ to the vibrational factor at $300 \mathrm{~K}$, which is less than the uncertainty of the procedure. Thus, for correct

http://www.splatalogue.net estimates of the column densities of the main isotopologue of glycolaldehyde the main species needs to be increased by a factor of 1.47 compared to estimates derived using the partition function given in the JPL catalogue.

The gas-phase infrared spectrum of ethylene glycol was published several years ago (Buckley \& Giguère 1967). Comparison with quantum-chemical calculations (Howard et al. 2005) suggests that all seven features measured below $1500 \mathrm{~K}$ are all fundamentals and that they may constitute all fundamentals below $1500 \mathrm{~K}$. These modes together yield a vibrational factor of 4.02. The contribution by the higher lying $g G g$ ' conformer should increase the total column density additionally by a factor of $\sim 1.38$ at $300 \mathrm{~K}$ (Müller \& Christen 2004).

\subsubsection{Line identification, temperatures, and column densities}

To identify the emission from each of the species the lines of glycolaldehyde and ethylene glycol are first identified in Band 3: the spectral windows in the $3 \mathrm{~mm}$ observations were selected to cover a number of the low excitation $v=0$ transitions of glycolaldehyde for which the frequencies had previously been measured in the laboratory. As these spectra are much less line confused than those at higher frequencies, it is meaningful to fit the individual lines. Table A.1 lists the parameters from Gaussian fits to the lines of glycolaldehyde as well as the two conformers of ethylene glycol in the central beam toward IRAS 16293B, and Fig. 9 shows rotation diagrams for these three species. If the emission of a set of lines is in local thermodynamic equilibrium and optically thin, the column density per statistical weight $N_{\mathrm{u}} / g_{\mathrm{u}}$ (directly derived from their measured line strengths) for the lines as function of their energies above the ground-state $E_{\text {up }}$ will follow the Boltzmann distribution. The total column density of the molecule and its kinetic temperature can thus be derived from linear fits to plots of $\ln \left(N_{\mathrm{u}} / g_{\mathrm{u}}\right)$ as a function of $E_{\mathrm{up}}$, where the column density can be derived as the intersect of the fit with the $Y$-axis and the temperature from the slope of the fit (e.g., Blake et al. 1987; Goldsmith et al. 1999). We used the parameters from these rotation diagram fits (Fig. 9) to create synthetic spectra for each of the observed spectral windows to strengthen the discoveries. Figures B.1, B.2 compare the model predictions to the full spectral ranges covered in the Band 3 and Band 6 parts of the survey, while Figs. C.2-C.4 show the observed and synthetic spectra for the 24 lines each of glycolaldehyde, $a G g^{\prime}$ and $g G g^{\prime}$ ethylene glycol, respectively, which are predicted to be the brightest in the Band 7 data while still having optical depths less than 0.2. A strong test of the assignments is that no lines are predicted to show emission at frequencies where none is seen in the spectra.

As can be observed from these fits, clear detections are seen of glycolaldehyde and the two conformers of ethylene glycol. The detection of ethylene glycol marks the first detection toward IRAS 16293-2422 and specifically the detection of the $g G g^{\prime}$ conformer (tentatively seen in the science verification data; Jørgensen et al. 2012) is the first solid detection of this conformer in the ISM at all. For glycolaldehyde and the lower state conformer of ethylene glycol, the $a G g^{\prime}$ conformer, good fits are obtained with rotation temperatures of approximately $300 \mathrm{~K}$, as was also found in the analysis of glycolaldehyde and methyl formate in the science verification data (Jørgensen et al. 2012). The fits for the higher state conformer of ethylene glycol, the $g G g^{\prime}$ conformer, has a lower rotation temperature, but the few lines for this species cover a rather limited range in $E_{\mathrm{u}}$ up to about $140 \mathrm{~K}$ and a rotation temperature of $300 \mathrm{~K}$, similar to that of the other conformer and glycolaldehyde, is consistent with 

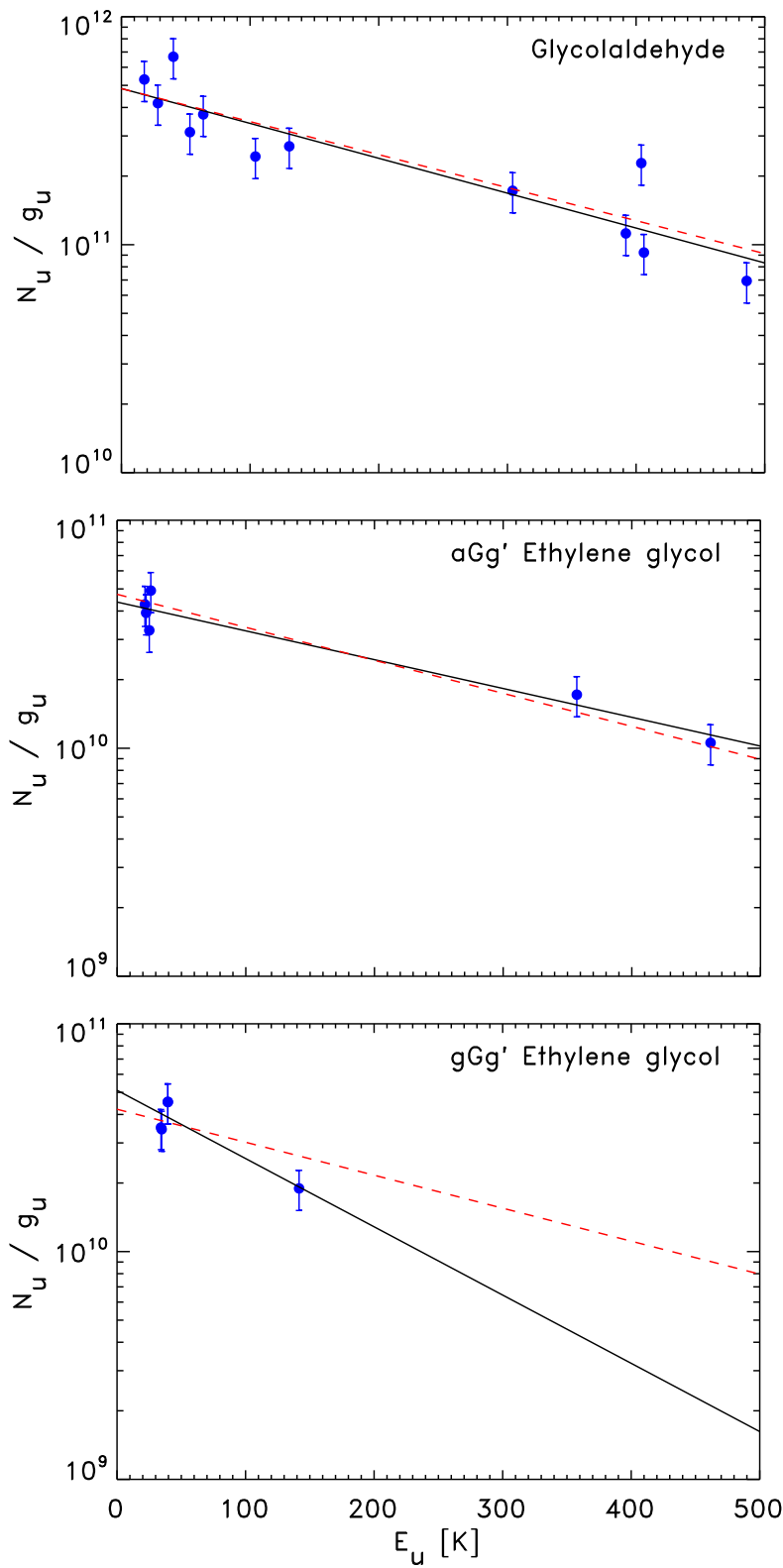

Fig. 9. Rotation diagrams for glycolaldehyde and the two conformers $\left(a G g^{\prime}\right.$ and $g G g^{\prime}$ ) of ethylene glycol based on the observations in Band 3 . The solid line indicates the best fit line to the rotation diagram for each species and the dashed red line the best fit for a fixed temperature of $300 \mathrm{~K}$. The error bars represent $1 \sigma$ uncertainties.

the data as well. Furthermore, when compared to the Band 6 and 7 data, it also appears that all lines are well reproduced with an excitation temperature of $300 \mathrm{~K}$. In particular, there are no systematics with either low- or high-excitation transitions being generally over- or under-produced, a clear indication that the excitation temperature of $300 \mathrm{~K}$ is a good approximation.

In terms of column densities, several complications arise when comparisons are made across the different wavelengths. Whereas the $3 \mathrm{~mm}$ transitions are all expected to be optically thin, both glycolaldehyde and ethylene glycol have lines at the higher frequencies becoming optically thick. This is partly an excitation effect and partly a result of the smaller beam and makes the column density estimates slightly more uncertain. Because of the differences in beam sizes between the Band 3 and Band 6/7 data, caution is necessary when deciding which beam filling factor to adopt for the analysis. Also, the optically thick continuum affects the observed line emission. Still, if only transitions predicted to have optical depths lower than 0.1 are considered, it seems that the best fit column densities are slightly lower for the two ethylene glycol conformers relative to glycolaldehyde at the higher frequencies in contrast to the case for the pure rotation diagram fits to the Band 3 data where the three species have similar column densities within the uncertainties. A possible explanation for this may be that ethylene glycol is slightly more extended around the protostar itself than glycolaldehyde - thus contributing more in the larger beam at $3 \mathrm{~mm}$ - while also being more obscured by the optically thick continuum in the Band 7 data. Hints of this are seen in the maps of the representative transitions in Fig. 7, but higher resolution imaging at lower frequencies as well as observations of isotopologues of ethylene glycol would be important to test whether line optical thickness or the distribution is more important. No strong lines of acetic acid are covered in the Band 3 data, but it is clearly seen in the Band 6 and 7 datasets (see Figs. B.1, B.2 and also Fig. C.5). To model the emission, the same excitation temperature as derived for the glycolaldehyde and ethylene glycol lines is adopted for the derivation of its column density using similar comparisons between observed and synthetic spectra as for $\mathrm{CH}_{3}^{18} \mathrm{OH}$.

\subsubsection{Comparison with models}

Figure 10 compares the derived column densities for methyl formate, glycolaldehyde, ethylene glycol, acetaldehyde, and acetic acid relative to methanol for IRAS 16293B, SgrB2(N) (Belloche et al. 2013), and three versions of the three-phase chemical models of hot cores by Garrod (2013). The models of Garrod simulate gas-phase, grain surface, and mantle chemistry during the formation of high-mass hot cores. The physical models represent the initial collapse followed by gradual warm-up of the gas and grains, the latter with three different rates, "slow" with warm-up to $200 \mathrm{~K}$ in $1 \times 10^{6} \mathrm{yr}$, "medium" reaching $200 \mathrm{~K}$ in $2 \times 10^{5} \mathrm{yr}$, and "fast" in $5 \times 10^{4} \mathrm{yr}$. The model values in Fig. 10 represent the peak fractional abundances for the individual species in these three model instances. The most notable aspect is the good agreement between the abundances relative to methanol for methyl formate, acetaldehyde, and acetic acid with those in $\operatorname{SgrB} 2(\mathrm{~N})$, but somewhat low abundances of glycolaldehyde and ethylene glycol. Taking into account both the $a G g^{\prime}$ and $g G g^{\prime}$ conformers, the ethylene glycol abundance is higher by about a factor of 3 than that of glycolaldehyde, in line with measurements for comets and the other solar-type protostar, NGC 1333-IRAS 2A where glycolaldehyde and the $a G g^{\prime}$ conformers have been detected (Coutens et al. 2015). This is in contrast to what was inferred based on the tentative detection of the $g G g^{\prime}$ conformer based on the SV data (Jørgensen et al. 2012). The reason for this discrepancy is that the analysis of the SV data did not take into account the vibrational correction factors for ethylene glycol and only considered one of the conformers. As noted above, these corrections are non-negligible at the temperature of $300 \mathrm{~K}$.

Generally, both observations and models find abundances for the five species relative to methanol in the few $0.01-1 \%$ ranges; however, some significant differences are seen within this range. For example, the models strongly overproduce the glycolaldehyde abundances relative to methyl formate when compared to the observations toward both IRAS $16293 \mathrm{~B}$ and SgrB2(N). This was also noted by Garrod (2013) who speculated that it was caused by differences in the formation efficiencies of methyl formate and glycolaldehyde through their primary grain-surface/ice 


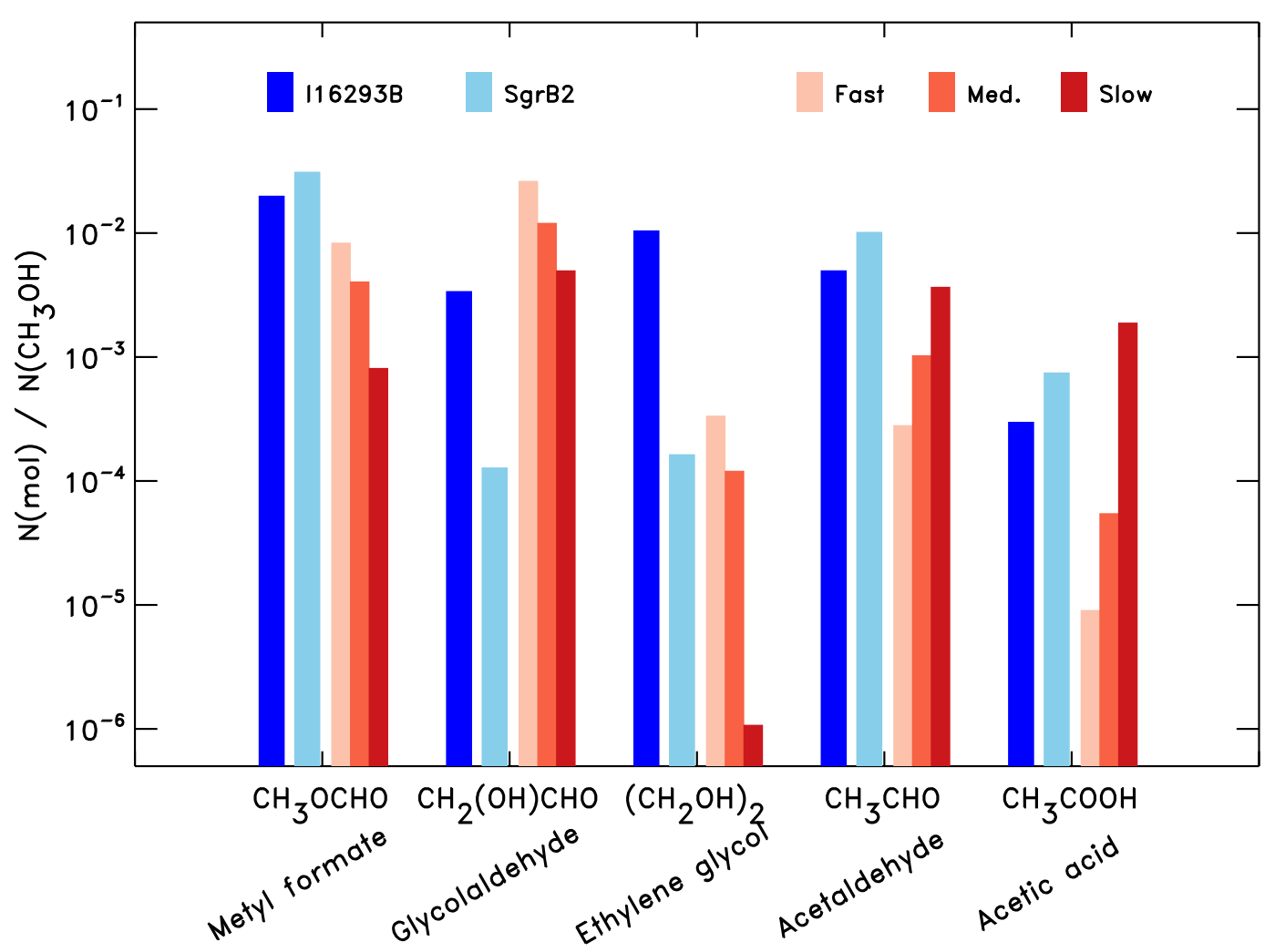

Fig. 10. Abundances of methyl formate $\left(\mathrm{CH}_{3} \mathrm{OCHO}\right)$, glycolaldehyde $\left(\mathrm{CH}_{2}(\mathrm{OH}) \mathrm{CHO}\right)$, ethylene glycol $\left(\left(\mathrm{CH}_{2} \mathrm{OH}\right)_{2}\right)$, acetaldehyde $\left(\mathrm{CH} \mathrm{CHO}_{3}\right)$, and acetic acid $\left(\mathrm{CH}_{3} \mathrm{COOH}\right)$ relative to methanol $\left(\mathrm{CH}_{3} \mathrm{OH}\right)$ toward IRAS 16293B (this paper) compared to SgrB2(N) from Belloche et al. (2013) and to the slow, medium, and fast models of Garrod (2013).

Table 3. Column densities of glycolaldehyde, the two conformers of ethylene glycol, acetic acid and methanol.

\begin{tabular}{llll}
\hline \hline Species & $T_{\text {rot }}{ }^{a}$ & $N(T=300 \mathrm{~K})^{b}$ & $N_{\text {synt }}{ }^{c}$ \\
\hline Glycolaldehyde & $284 \pm 27 \mathrm{~K}$ & $3.3 \times 10^{16} \mathrm{~cm}^{-2}$ & $6.8 \times 10^{16} \mathrm{~cm}^{-2}$ \\
$a G g^{\prime}$ ethylene glycol & $344 \pm 52 \mathrm{~K}$ & $3.3 \times 10^{16} \mathrm{~cm}^{-2}$ & $1.1 \times 10^{17} \mathrm{~cm}^{-2}$ \\
$g G g^{\prime}$ ethylene glycol & $145 \pm 45 \mathrm{~K}$ & $3.0 \times 10^{16} \mathrm{~cm}^{-2}$ & $1.0 \times 10^{17} \mathrm{~cm}^{-2}$ \\
Acetic acid $^{d}$ & $\ldots$ & $\ldots$ & $6 \times 10^{15} \mathrm{~cm}^{-2}$ \\
Methanol $^{e}$ & $\ldots$ & $\ldots$ & $2 \times 10^{19} \mathrm{~cm}^{-2}$ \\
\hline
\end{tabular}

Notes. ${ }^{(a)}$ Derived rotation temperature from fit to Band 3 data (Fig. 9). ${ }^{(b)}$ Column densities from Band 3 data assuming that $T=300 \mathrm{~K}$ and that the emission is uniformly extended compared to the beam. ${ }^{(c)}$ Column density from fits of synthetic spectra to the Band 7 data assuming a source size of $0.5^{\prime \prime}$ (see also Lykke et al., in prep.) and including vibrational corrections. ${ }^{(d)}$ From fitting synthetic spectra to the Band 6 and 7 data (Figs. B.1-B.2 and Fig. C.5) adopting an excitation temperature of $300 \mathrm{~K} .{ }^{(e)}$ Derived by fitting synthetic spectra of $\mathrm{CH}_{3}^{18} \mathrm{OH}$ in $\mathrm{Band} 7$ as discussed in Sect. 5.1.

formation routes involving $\mathrm{HCO}$ and respectively $\mathrm{CH}_{3} \mathrm{O}$ and $\mathrm{CH}_{2} \mathrm{OH}$ for methyl formate and glycolaldehyde. The models of Garrod (2013) require photodissociation in the ices to lead to those radicals, but recent experiments (Fedoseev et al. 2015; Chuang et al. 2016) show that methyl formate, glycolaldehyde, and ethylene glycol can also be formed in ices at low temperatures through $\mathrm{H}$-atom addition and abstraction reactions during the formation of $\mathrm{CH}_{3} \mathrm{OH}$ from $\mathrm{CO}$ and $\mathrm{H}_{2} \mathrm{CO}$. These reactions also make the link between glycolaldehyde and ethylene glycol stronger, which possibly accounts for the higher abundances of these species relative to methyl formate between IRAS 16293B on one side and SgrB2(N) on the other.

Concerning acetic acid and acetaldehyde, the agreement between the IRAS 16293B and SgrB2(N) abundances is noteworthy. Although acetic acid is an isomer of methyl formate and glycolaldehyde, its formation is not expected to follow the same paths owing to its different structure (Garrod 2013). As those models show, the resulting abundances are strongly dependent on the warm-up timescale with the slower models allowing for more build-up of acetic acid through the addition of $\mathrm{OH}$ to $\mathrm{CH}_{3} \mathrm{CO}$, which has been derived through hydrogen abstraction of acetaldehyde $\left(\mathrm{CH}_{3} \mathrm{CHO}\right)$. There is, however, a priori no reason why the warm-up rates should be similar in IRAS 16293B and SgrB2(N), so some other parameters must come into play and serve to regulate the abundances.

\subsection{Isotopologues of glycolaldehyde}

A potentially very intriguing possibility offered by ALMA is to characterize the isotopic composition of different complex organics and to use the possible differences to show the relationships between different complex organics. 
Charnley et al. (2004), for example, proposed a number of tests of the origins of typical hot core organics by measuring their ${ }^{12} \mathrm{C} /{ }^{13} \mathrm{C}$ fractionation patterns in different functional groups. Another interesting culprit is the $\mathrm{D} / \mathrm{H}$ ratio for different complex organics: Chemical models predict that the $\mathrm{D} / \mathrm{H}$ ratios for different species reflect the conditions at the time of their formation (e.g., Cazaux et al. 2011; Taquet et al. 2013) and, consequently, the formation pathways for different complex organics may thereby lead to different levels of deuteration (e.g., Taquet et al. 2014). These effects may, for example, explain the differences in the $\mathrm{D} / \mathrm{H}$ ratio of water and methanol with the former formed earlier in the evolution of prestellar cores where the $\mathrm{D} / \mathrm{H}$ ratio is lower (see Ceccarelli et al. 2014, for a discussion).

To start the fractionation study in the PILS data, we searched for the ${ }^{13} \mathrm{C}$ - and deuterated isotopologues for glycolaldehyde in the Band 7 data. Similar to the approach above we generate synthetic spectra and match those with the lines predicted to be the brightest in the spectra. The spectroscopy for the isotopologues is available through the CDMS database: for the two ${ }^{13} \mathrm{C}$-isotopologues of glycolaldehyde, ${ }^{13} \mathrm{CH}_{2} \mathrm{OHCHO}$ and $\mathrm{CH}_{2} \mathrm{OH}^{13} \mathrm{CHO}$, the data are based on measurements by Haykal et al. (2013), while those for the three deuterated isotopologues, $\mathrm{CHDOHCHO}, \mathrm{CH}_{2} \mathrm{ODCHO}$, and $\mathrm{CH}_{2} \mathrm{OHCDO}$, are based on Bouchez et al. (2012). The partition function for the isotopologues listed by the CDMS do not include the corrections for the vibrational contributions. Information on vibrational states of isotopic species have not been published experimentally, but quantum-chemical calculations on the main and the OD species are available, as is an investigation of the anharmonicity of the lowest two vibrational states $v_{18}=1$ and $v_{17}=1$ (Senent 2004). Scaling the lowest six vibrational modes by the calculated D-to-H ratio yields a vibrational factor of 3.29 for the OD species. The increase is mainly due to the isotopic shift of the $v_{17}$ mode. Vibrational factors of all other isotopologues are probably closer to the value of the main isotopologue because the lowest three vibrations involve mainly the heavy atoms or the $\mathrm{H}$ on the $\mathrm{OH}$ group ( $v_{17}$ mainly). In summary, the column density of the OD isotopologue needs to be increased by 3.29 and those of all other isotopologues by 2.80 to account for the population of vibrational states at $300 \mathrm{~K}$.

Figures C.6, C.7 show the spectra for the lines predicted to be the brightest for each of the deuterated isotopologues; for these species lines are clearly detected throughout the band. The lines of the ${ }^{13} \mathrm{C}$ species are intrinsically weaker and line blending becomes more significant. A number of reasonable assignments can still be made, which are compiled in Fig. C.8. In these plots only bright and/or relatively isolated lines are shown, corresponding to about half of the transitions predicted to be the brightest from synthetic spectra at $300 \mathrm{~K}$. The derived column densities are given in Table 4. For the deuterated species the immediately derived column density of the isotopologue with the deuteration in the $\mathrm{CH}_{2}$ group is a factor two higher than that of the other two isotopologues. This should be expected statistically if the $\mathrm{D} / \mathrm{H}$ ratio is similar for each of the hydrogen atoms across the functional groups (the $\mathrm{CHDOHCHO}$ and $\mathrm{CDHOHCHO}$ versions being indistinguishable). Taking this into account, the $\mathrm{D} / \mathrm{H}$ ratio for glycolaldehyde is $4.1-5.2 \%$, where the differences between the deuteration of the different functional groups appear marginal given the uncertainties in the line identification and modeling. For the ${ }^{13} \mathrm{C}$ species, the uncertainties are somewhat larger owing to the smaller number of lines, but the modeling of both species appears consistent with a column density of $1.9-2.8 \times 10^{15} \mathrm{~cm}^{-2}$ or a ${ }^{12} \mathrm{C} /{ }^{13} \mathrm{C}$ ratio of $24-35$, with a best fit column density of $2.5 \times 10^{15} \mathrm{~cm}^{-2}$ fitting the two species
Table 4. Derived column densities for the isotopologues of glycolaldehyde and abundances relative to the main isotopologue.

\begin{tabular}{lll}
\hline \hline Species & $\begin{array}{l}N^{a} \\
{\left[\mathrm{~cm}^{-2}\right]}\end{array}$ & $N / N\left(\mathrm{CH}_{2} \mathrm{OHCHO}\right)^{b}$ \\
\hline $\mathrm{CH}_{2} \mathrm{OHCHO}$ & $6.8 \times 10^{16}$ & $\ldots$ \\
$\mathrm{CHDOHCHO}$ & $7.1 \times 10^{15}$ & $0.10(9.6)$ \\
$\mathrm{CH}_{2} \mathrm{ODCHO}$ & $3.2 \times 10^{15}$ & $0.047(21)$ \\
$\mathrm{CH}_{2} \mathrm{OHCDO}$ & $3.5 \times 10^{15}$ & $0.052(19)$ \\
${ }^{13} \mathrm{CH}_{2} \mathrm{OHCHO}$ & $2.5 \times 10^{15 c}$ & $0.037(27)^{c}$ \\
$\mathrm{CH}_{2} \mathrm{OH}^{13} \mathrm{CHO}$ & $2.5 \times 10^{15 c}$ & $0.037(27)^{c}$ \\
\hline
\end{tabular}

Notes. ${ }^{(a)}$ Column density of the isotopologue corrected for the vibrational contributions at $300 \mathrm{~K} .{ }^{(b)}$ Column density of isotopologue relative to that of the main species. The number in parentheses gives the inverse ratio: traditionally the first number is quoted for measurements of the deuterated species, while the number in parentheses is typically quoted for ${ }^{12} \mathrm{C} /{ }^{13} \mathrm{C}$ measurements. ${ }^{(c)}$ The ${ }^{13} \mathrm{CH}_{2} \mathrm{OHCHO}$ and $\mathrm{CH}_{2} \mathrm{OH}^{13} \mathrm{CHO}$ fitted with a common column density for both owing to the relatively low number of lines seen for the two species.

simultaneously. It is worth emphasizing that these detections illustrate the great sensitivity of the ALMA observations, allowing us to measure emission from species with abundances below $0.01 \%$ with respect to methanol $\left(\mathrm{CH}_{3} \mathrm{OH}\right)$.

The $\mathrm{D} / \mathrm{H}$ ratio for glycolaldehyde of $\approx 5 \%$ is clearly higher than the typical water deuteration levels of $\approx 0.1 \%$ in the warm gas on the same scales probed by interferometric observations toward IRAS 16293-2422 (e.g., Persson et al. 2013) and other protostars (e.g., Jørgensen \& van Dishoeck 2010; Taquet et al. 2013; Persson et al. 2014). It appears to be lower than the value inferred for formaldehyde, methanol, and other complex organics from single-dish observations of IRAS 16293-2422 and other protostars (e.g., Parise et al. 2006; Demyk et al. 2010; Richard et al. 2013). Before concluding that this lower ratio reflects differences in the processes involved in the formation of glycolaldehyde, caution must be taken as the single-dish and interferometric data probe very different environments owing to the very different beam sizes. The significant beam dilution and excitation/optical depth effects may also be an issue for the single-dish observations where many components are enclosed in one beam. Clearly, a systematic comparison of the $\mathrm{D} / \mathrm{H}$ ratios for complex organics on the scales of the ALMA observations will be critical.

It is particularly noteworthy that no differences are seen in the deuteration of the three different functional groups in glycolaldehyde. For deuterated methanol the $\mathrm{CH}_{3}$-group has been found to be significantly enhanced compared to the $\mathrm{OH}$ group with the $\mathrm{D} / \mathrm{H}$ ratios of $12 \%$ for $\mathrm{CH}_{2} \mathrm{DOH}$ (ratio of the column densities of $\mathrm{CH}_{2} \mathrm{DOH} / \mathrm{CH}_{3} \mathrm{OH}$ of $37 \%$ ) and $1.8 \%$ for $\mathrm{CH}_{3} \mathrm{OD}$ (Parise et al. 2004). This led Faure et al. (2015) to propose that the functional groups able to establish hydrogen bonds $(-\mathrm{OH}$, $-\mathrm{NH})$ are expected to equilibrate with the $\mathrm{D} / \mathrm{H}$ ratio in water ice during heating events. In this scenario, the $\mathrm{OH}$ group of glycolaldehyde should show a significantly lower $\mathrm{D} / \mathrm{H}$ ratio than the $\mathrm{CH}_{2}$ and $\mathrm{CHO}$ groups, in contrast to what is observed. A separate analysis of formamide, $\mathrm{NH}_{2} \mathrm{CHO}$, based on the PILS data (Coutens et al. 2016) shows a similar effect with the three deuterated isotopologues $\mathrm{NH}_{2} \mathrm{CDO}$ and cis-/trans-NHDCHO all having similar degrees of deuteration. 
The inferred ${ }^{12} \mathrm{C} /{ }^{13} \mathrm{C}$ ratio of $24-35$ is lower than the ratio of $68 \pm 15$ of the local interstellar medium (Milam et al. 2005). This appears to be a real difference and does not reflect, e.g., high optical depth of the lines of the main isotopologue given that a relatively high number of significant, low optical depth lines have been identified. It is in contrast to recent measurements of the ${ }^{12} \mathrm{C} /{ }^{13} \mathrm{C}$ ratio for methyl formate toward a sample of high-mass star-forming regions (including Orion-KL) by Favre et al. (2014a), who found isotope ratios for complex organic molecules similar to the value for $\mathrm{CO}$. The ${ }^{13} \mathrm{CO}$ enhancement could result from low temperature ion-molecule reactions in the gas increasing the gaseous ${ }^{13} \mathrm{CO}$ abundance at $10 \mathrm{~K}$ just prior to freeze-out (Langer et al. 1984). Once on the grains, the enhanced ${ }^{13} \mathrm{CO}$ can be further incorporated into complex organic molecules (Charnley et al. 2004). This could be a simple explanation for the measured ratio for glycolaldehyde: if formed efficiently from $\mathrm{CO}$ ices at low temperatures $(T \approx 10-15 \mathrm{~K})$ on the grains, as has been proposed by Fedoseev et al. (2015) and Chuang et al. (2016) based on laboratory experiments, glycolaldehyde ice would naturally inherit any ${ }^{13} \mathrm{C}$-enhancement (i.e., low ${ }^{12} \mathrm{C}:{ }^{13} \mathrm{C}$ isotopologue ratio) from the $\mathrm{CO}$ ices, which would be preserved upon sublimation into the gas-phase. In that case many of the other complex organics formed at low temperatures in the ices from $\mathrm{CO}$, should show low ratios between the ${ }^{12} \mathrm{C}$ and ${ }^{13} \mathrm{C}$ isotopologues that are comparable to glycolaldehyde, whereas molecules with significant contributions from other $\mathrm{C}$-containing radicals or from gas-phase routes may not (Charnley et al. 2004). The more "standard" ${ }^{12} \mathrm{C}:{ }^{13} \mathrm{C}$ ratio in the high-mass star-forming regions would then reflect the shorter timescales and higher temperatures there, with $13 \mathrm{CO}$ less enhanced, similar to what has been invoked to explain the differences in the deuteration.

An alternative is that the ${ }^{12} \mathrm{CO} /{ }^{13} \mathrm{CO}$ ratio in the ices is affected by slightly different binding energies of the two isotopologues. Smith et al. (2015) recently measured the ${ }^{12} \mathrm{CO} /{ }^{13} \mathrm{CO}$ ratio in the gas-phase for a sample of young stellar objects (including four in Ophiuchus) through high spectral resolution infrared observations and generally found very high ${ }^{12} \mathrm{CO} /{ }^{13} \mathrm{CO}$ values ranging from 85 to 165 . Smith et al. discuss several options for these high gas-phase values, including possible differential sublimation of ${ }^{12} \mathrm{CO}$ versus ${ }^{13} \mathrm{CO}$ due to slightly different binding energies and isotope selective photodissociation coupled with low temperature chemistry. It should be noted that no strong evidence for such differences in binding energies has yet been found in the laboratory experiments (e.g., Bisschop et al. 2006; Acharyya et al. 2007). However, if the binding energies were in fact different, it would lead ${ }^{12} \mathrm{CO}$ to sublimate at slightly lower temperatures than ${ }^{13} \mathrm{CO}$, thus leading to an enhancement of the ${ }^{13} \mathrm{C}$-isotopologue in the ice and, vice versa, an enhancement of the ${ }^{12} \mathrm{C}$-isotopologue in the gas-phase. Further observations of the carbon-isotopic composition of complex organics could shed more light on the underlying mechanism and further extend its use in determining the formation routes for the different complex organics.

\section{Summary}

We have presented an overview and some of the first results from a large unbiased spectral survey of the protostellar binary IRAS 16293-2422 using ALMA. The full frequency window from 329 to $363 \mathrm{GHz}$ is covered with a spectral resolution of $0.2 \mathrm{~km} \mathrm{~s}^{-1}$ and beam size of $0.5^{\prime \prime}$ (60 AU diameter at the distance of IRAS 16293-2422) in addition to three selected settings at 3.0 and $1.3 \mathrm{~mm}$. The main findings of this paper are as follows:

- The continuum is well detected in each band with different signatures toward the two protostars: clear elongated emission seen toward IRAS 16293A in the direction of a previously reported velocity gradient, making it appear as a flattened edge-on structure. The binarity of this source at submillimeter wavelengths previously reported is not confirmed in these data. In contrast, the emission for IRAS $16293 \mathrm{~B}$ is clearly optically thick out to wavelengths of approximately $1 \mathrm{~mm}$ (and possibly beyond). The optical thickness of radiation toward IRAS 16293B confirms that the emission has its origin in a different component than the larger scale envelope with a density larger than $3 \times 10^{10} \mathrm{~cm}^{-3}$.

- More than 10000 lines are seen in spectra toward IRAS 16293B. The high gas density implied by the continuum radiation means that lines of typical complex organic molecules are thermalized and LTE is thus a valid approximation for their analysis.

- The spectra at 3.0, 1.3, and $0.8 \mathrm{~mm}$ provide strong confirmation of the previous detection of glycolaldehyde and of the derived excitation temperature of $300 \mathrm{~K}$ based on ALMA Science Verification data (Jørgensen et al. 2012). In addition, the spectra show detections of acetic acid (isomer of glycolaldehyde) and two conformers of ethylene glycol (the reduced alcohol of glycolaldehyde); the detection of the $g G g^{\prime}$ conformer is the first reported detection in the ISM. The excitation temperatures of these species are consistent, $\approx 300 \mathrm{~K}$. The abundance of glycolaldehyde is comparable to that of the main conformer of ethylene glycol with the second conformer not much rarer, as one would expect given the high temperatures in the gas. Small differences between the relative glycolaldehyde and ethylene glycol abundances in the data of different beam sizes possibly reflect glycolaldehyde being slightly more centrally concentrated than ethylene glycol.

- Relative to methanol (determined from observations of optically thin lines of the $\mathrm{CH}_{3}^{18} \mathrm{OH}$ isotopologue), the abundances of glycolaldehyde and related species are between $0.03 \%$ and $\approx 1 \%$. Glycolaldehyde and ethylene glycol are clearly more abundant relative to methanol toward IRAS 16293B compared to the Galactic Center source SgrB2(N) (Belloche et al. 2013), whereas the abundances of the glycolaldehyde isomers (methyl formate and acetic acid) and acetaldehyde are comparable for the two sources. A possible explanation for this is the formation of glycolaldehyde from $\mathrm{CO}$ at low temperatures in ices toward IRAS 16293-2422, in agreement with recent laboratory experiments, a route that is unlikely to apply for the warmer Galactic Center.

- The data also show detections of two ${ }^{13} \mathrm{C}$-isotopologues of glycolaldehyde as well as three deuterated isotopologues. These are the first detections of these five isotopologues reported for the ISM, enabled by the narrow line widths toward IRAS $16293 \mathrm{~B}$. The $\mathrm{D} / \mathrm{H}$ ratio for glycolaldehyde is $\approx 5 \%$ for all three deuterated isotopologues with no measurable differences for the deuteration of the different functional groups. These ratios are higher than in water, but lower than reported $\mathrm{D} / \mathrm{H}$ ratios for methanol, formaldehyde, and other complex organics, although those should be revisited at the same scales. The ${ }^{12} \mathrm{C} /{ }^{13} \mathrm{C}$ ratio of glycolaldehyde of $\approx 30$ derived from our data is lower than the canonical ISM. 
This may reflect a low ${ }^{12} \mathrm{CO} /{ }^{13} \mathrm{CO}$ ratio in the ice from which it is formed, either owing to ion-molecule reactions in the gas or differences in binding energies for the different $\mathrm{CO}$ isotopologues.

This first presentation of data has only scratched the surface of all the information available in the survey, but has already raised a number of new questions concerning, in particular, the formation of complex organic molecules around protostars. Moving forward, it is clear that the possibility to systematically derive accurate relative abundances of different organic molecules (and their isotopologues) will be an important tool. In this respect, many of the answers to the questions concerning the origin of complex prebiotic molecules may be hidden in this rich ALMA dataset.

Acknowledgements. We are grateful to the staff at the Nordic ALMA Regional Center (ARC) node at Onsala Space Observatory, in particular, Ivan Martí-Vidal and Sébastien Muller, for their support in setting up the program and for assistance with the data reduction. We also thank the anonymous referee for many comments that helped improve the presentation Thanks also go to G. Fedoseev, K.-J. Chuang, and H. Linnartz for experimental information and discussions on the formation of glycolaldehyde and ethylene glycol in ices at low temperatures. This paper make use of the following ALMA data: ADS/JAO.ALMA\#2012.1.00712.S and ADS/JAO.ALMA\#2013.1.00278.S. ALMA is a partnership of ESO (representing its member states), NSF (USA) and NINS (Japan), together with NRC (Canada), NSC and ASIAA (Taiwan), and KASI (Republic of Korea), in cooperation with the Republic of Chile. The Joint ALMA Observatory is operated by ESO, AUI/NRAO and NAOJ. The group of J.K.J. acknowledges support from a Lundbeck Foundation Group Leader Fellowship as well as the European Research Council (ERC) under the European Union's Horizon 2020 research and innovation programme (grant agreement No. 646908) through ERC Consolidator Grant "S4F". Research at the Centre for Star and Planet Formation is funded by the Danish National Research Foundation. The work of AC was funded by a STFC grant. AC thanks the COST action CM1401 "Our Astrochemical History" for additional financial support. The group of EvD acknowledges A-ERC grant 291141 CHEMPLAN.

\section{References}

Acharyya, K., Fuchs, G. W., Fraser, H. J., van Dishoeck, E. F., \& Linnartz, H 2007, A\&A, 466, 1005

Adams, F. C., \& Shu, F. H. 1986, ApJ, 308, 836

André, P., Ward-Thompson, D., \& Barsony, M. 1993, ApJ, 406, 122

Arce, H. G., Santiago-García, J., Jørgensen, J. K., Tafalla, M., \& Bachiller, R. 2008, ApJ, 681, L21

Bachiller, R., \& Pérez Gutiérrez, M. 1997, ApJ, 487, L93

Bacmann, A., Caux, E., Hily-Blant, P., et al. 2010, A\&A, 521, L42

Bacmann, A., Taquet, V., Faure, A., Kahane, C., \& Ceccarelli, C. 2012, A\&A, 541, L12

Baryshev, A. M., Hesper, R., Mena, F. P., et al. 2015, A\&A, 577, A129

Belloche, A., Müller, H. S. P., Menten, K. M., Schilke, P., \& Comito, C. 2013 A\&A, 559, A47

Belloche, A., Müller, H. S. P., Garrod, R. T., \& Menten, K. M. 2016, A\&A, 587, A91

Beltrán, M. T., Codella, C., Viti, S., Neri, R., \& Cesaroni, R. 2009, ApJ, 690, L93

Bisschop, S. E., Fraser, H. J., Öberg, K. I., van Dishoeck, E. F., \& Schlemmer, S. 2006, A\&A, 449, 1297

Bisschop, S. E., Jørgensen, J. K., van Dishoeck, E. F., \& de Wachter, E. B. M. 2007, A\&A, 465, 913

Bisschop, S. E., Jørgensen, J. K., Bourke, T. L., Bottinelli, S., \& van Dishoeck, E. F. 2008, A\&A, 488, 959

Biver, N., Bockelée-Morvan, D., Debout, V., et al. 2014, A\&A, 566, L5

Biver, N., Bockelee-Morvan, D., Moreno, R., et al. 2015, Sci. Adv., 1, e1500863

Blake, G. A., Sutton, E. C., Masson, C. R., \& Phillips, T. G. 1987, ApJ, 315, 62

Blake, G. A., van Dishoek, E. F., Jansen, D. J., Groesbeck, T. D., \& Mundy, L. G. 1994, ApJ, 428, 680

Bottinelli, S., Ceccarelli, C., Lefloch, B., et al. 2004a, ApJ, 615, 354

Bottinelli, S., Ceccarelli, C., Neri, R., et al. 2004b, ApJ, 617, L69

Bottinelli, S., Wakelam, V., Caux, E., et al. 2014, MNRAS, 441, 1964

Bouchez, A., Margulès, L., Motiyenko, R. A., et al. 2012, A\&A, 540, A51
Brogan, C. L., Pérez, L. M., et al. (ALMA Partnership) 2015, ApJ, 808, L3 Brouillet, N., Despois, D., Lu, X.-H., et al. 2015, A\&A, 576, A129 Brown, R. D., Crofts, J. G., Godfrey, P. D., et al. 1975, ApJ, 197, L29 Buckley, P., \& Giguère, P. A. 1967, Can. J. Chem., 45, 397 Butler, R. A. H., De Lucia, F. C., Petkie, D. T., et al. 2001, ApJS, 134, 319 Butner, H. M., Charnley, S. B., Ceccarelli, C., et al. 2007, ApJ, 659, L137 Calcutt, H., Viti, S., Codella, C., et al. 2014, MNRAS, 443, 3157

Carroll, P. B., Drouin, B. J., \& Widicus Weaver, S. L. 2010, ApJ, 723, 845

Caselli, P., van der Tak, F. F. S., Ceccarelli, C., \& Bacmann, A. 2003, A\&A, 403, L37

Castets, A., Ceccarelli, C., Loinard, L., Caux, E., \& Lefloch, B. 2001, A\&A, 375, 40

Caux, E., Kahane, C., Castets, A., et al. 2011, A\&A, 532, A23

Cazaux, S., Tielens, A. G. G. M., Ceccarelli, C., et al. 2003, ApJ, 593, L51

Cazaux, S., Caselli, P., \& Spaans, M. 2011, ApJ, 741, L34

Ceccarelli, C., Castets, A., Loinard, L., Caux, E., \& Tielens, A. G. G. M. 1998a, A\&A, 338, L43

Ceccarelli, C., Caux, E., White, G. J., et al. 1998b, A\&A, 331, 372

Ceccarelli, C., Caux, E., Loinard, L., et al. 1999, A\&A, 342, L21

Ceccarelli, C., Castets, A., Caux, E., et al. 2000a, A\&A, 355, 1129

Ceccarelli, C., Loinard, L., Castets, A., Faure, A., \& Lefloch, B. 2000b, A\&A, 362,1122

Ceccarelli, C., Loinard, L., Castets, A., Tielens, A. G. G. M., \& Caux, E. 2000c, A\&A, 357, L9

Ceccarelli, C., Loinard, L., Castets, A., et al. 2001, A\&A, 372, 998

Ceccarelli, C., Maret, S., Tielens, A. G. G. M., Castets, A., \& Caux, E. 2003, A\&A, 410, 587

Ceccarelli, C., Bacmann, A., Boogert, A., et al. 2010, A\&A, 521, L22

Ceccarelli, C., Caselli, P., Bockelée-Morvan, D., et al. 2014, Protostars and Planets VI, 859

Chandler, C. J., Brogan, C. L., Shirley, Y. L., \& Loinard, L. 2005, ApJ, 632, 371

Charnley, S. B., Ehrenfreund, P., Millar, T. J., et al. 2004, MNRAS, 347, 157

Christen, D., \& Müller, H. S. P. 2003, Phys. Chem. Chem. Phys., 5, 3600

Chuang, K.-J., Fedoseev, G., Ioppolo, S., van Dishoeck, E. F., \& Linnartz, H. 2016, MNRAS, 455, 1702

Codella, C., Cabrit, S., Gueth, F., et al. 2014, A\&A, 568, L5

Correia, J. C., Griffin, M., \& Saraceno, P. 2004, A\&A, 418, 607

Coutens, A., Vastel, C., Caux, E., et al. 2012, A\&A, 539, A132

Coutens, A., Vastel, C., Cazaux, S., et al. 2013, A\&A, 553, A75

Coutens, A., Persson, M. V., Jørgensen, J. K., Wampfler, S. F., \& Lykke, J. M. 2015, A\&A, 576, A5

Coutens, A., Jørgensen, J. K., van der Wiel, M. H. D., et al. 2016, A\&A, 590, L6

Crovisier, J., Bockelée-Morvan, D., Biver, N., et al. 2004, A\&A, 418, L35

Demyk, K., Bottinelli, S., Caux, E., et al. 2010, A\&A, 517, A17

Doty, S. D., Schöier, F. L., \& van Dishoeck, E. F. 2004, A\&A, 418, 1021

Ellder, J., Friberg, P., Hjalmarson, A., et al. 1980, ApJ, 242, L93

Faure, A., Faure, M., Theulé, P., Quirico, E., \& Schmitt, B. 2015, A\&A, 584, A98

Favre, C., Carvajal, M., Field, D., et al. 2014a, ApJS, 215, 25

Favre, C., Jørgensen, J. K., Field, D., et al. 2014b, ApJ, 790, 55

Fedoseev, G., Cuppen, H. M., Ioppolo, S., Lamberts, T., \& Linnartz, H. 2015, MNRAS, 448, 1288

Fraser, H. J., Collings, M. P., McCoustra, M. R. S., \& Williams, D. A. 2001, MNRAS, 327, 1165

Friesen, R. K., Di Francesco, J., Bourke, T. L., et al. 2014, ApJ, 797, 27

Fuente, A., Cernicharo, J., Caselli, P., et al. 2014, A\&A, 568, A65

Fukui, Y., Sugitani, K., Takaba, H., et al. 1986, ApJ, 311, L85

Furuya, R. S., Kitamura, Y., Wootten, H. A., Claussen, M. J., \& Kawabe, R. 2001, ApJ, 559, L143

Garay, G., Mardones, D., Rodríguez, L. F., Caselli, P., \& Bourke, T. L. 2002, ApJ, 567, 980

Garrod, R. T. 2013, ApJ, 765, 60

Gerin, M., Lis, D. C., Philipp, S., et al. 2006, A\&A, 454, L63

Gibb, E., Nummelin, A., Irvine, W. M., Whittet, D. C. B., \& Bergman, P. 2000, ApJ, 545, 309

Girart, J. M., Estalella, R., Palau, A., Torrelles, J. M., \& Rao, R. 2014, ApJ, 780, L11

Goldsmith, P. F., Langer, W. D., \& Velusamy, T. 1999, ApJ, 519, L173

Halfen, D. T., Apponi, A. J., Woolf, N., Polt, R., \& Ziurys, L. M. 2006, ApJ, 639, 237

Haykal, I., Motiyenko, R. A., Margulès, L., \& Huet, T. R. 2013, A\&A, 549, A96

Herbst, E., \& van Dishoeck, E. F. 2009, ARA\&A, 47, 427

Hily-Blant, P., Maret, S., Bacmann, A., et al. 2010, A\&A, 521, L52

Hirano, N., Mikami, H., Umemoto, T., Yamamoto, S., \& Taniguchi, Y. 2001, ApJ, 547, 899

Hollis, J. M., Lovas, F. J., \& Jewell, P. R. 2000, ApJ, 540, L107 
Hollis, J. M., Vogel, S. N., Snyder, L. E., Jewell, P. R., \& Lovas, F. J. 2001, ApJ, 554, L81

Hollis, J. M., Lovas, F. J., Jewell, P. R., \& Coudert, L. H. 2002, ApJ, 571, L59

Hollis, J. M., Jewell, P. R., Lovas, F. J., \& Remijan, A. 2004, ApJ, 613, L45

Howard, D. L., Jørgensen, P., \& Kjaergaard, H. G. 2005, J. A. Chem. Soc., 127 17096

Ilyushin, V., Kryvda, A., \& Alekseev, E. 2009, J. Mol. Spectr., 255, 32

Jaber, A. A., Ceccarelli, C., Kahane, C., \& Caux, E. 2014, ApJ, 791, 29

Johnson, T. J., Sams, R. L., Profeta, L. T. M., et al. 2013, J. Phys. Chem. A, 117 4096

Jørgensen, J. K., \& van Dishoeck, E. F. 2010, ApJ, 725, L172

Jørgensen, J. K., Schöier, F. L., \& van Dishoeck, E. F. 2002, A\&A, 389, 908

Jørgensen, J. K., Hogerheijde, M. R., van Dishoeck, E. F., Blake, G. A., \& Schöier, F. L. 2004, A\&A, 413, 993

Jørgensen, J. K., Bourke, T. L., Myers, P. C., et al. 2005a, ApJ, 632, 973

Jørgensen, J. K., Lahuis, F., Schöier, F. L., et al. 2005b, ApJ, 631, L77

Jørgensen, J. K., Schöier, F. L., \& van Dishoeck, E. F. 2005c, A\&A, 437, 501

Jørgensen, J. K., Johnstone, D., Kirk, H., et al. 2008, ApJ, 683, 822

Jørgensen, J. K., Bourke, T. L., Nguyen Luong, Q., \& Takakuwa, S. 2011, A\&A, 534, A 100

Jørgensen, J. K., Favre, C., Bisschop, S. E., et al. 2012, ApJ, 757, L4

Jørgensen, J. K., Visser, R., Sakai, N., et al. 2013, ApJ, 779, L22

Kahane, C., Ceccarelli, C., Faure, A., \& Caux, E. 2013, ApJ, 763, L38

Knude, J., \& Høg, E. 1998, A\&A, 338, 897

Kristensen, L. E., Klaassen, P. D., Mottram, J. C., Schmalzl, M., \& Hogerheijde, M. R. 2013, A\&A, 549, L6

Kuan, Y., Huang, H., Charnley, S. B., et al. 2004, ApJ, 616, L27

Lada, C. J., \& Wilking, B. A. 1984, ApJ, 287, 610

Langer, W. D., Graedel, T. E., Frerking, M. A., \& Armentrout, P. B. 1984, ApJ, 277,581

Lindberg, J. E., Jørgensen, J. K., Brinch, C., et al. 2014, A\&A, 566, A74

Lis, D. C., Gerin, M., Roueff, E., Vastel, C., \& Phillips, T. G. 2006, ApJ, 636, 916

Loinard, L., Castets, A., Ceccarelli, C., et al. 2000, A\&A, 359, 1169

Loinard, L., Castets, A., Ceccarelli, C., Caux, E., \& Tielens, A. G. G. M. 2001 ApJ, 552, L163

Loinard, L., Chandler, C. J., Rodríguez, L. F., et al. 2007, ApJ, 670, 1353

Loinard, L., Torres, R. M., Mioduszewski, A. J., \& Rodríguez, L. F. 2008, ApJ, 675, L29

Loinard, L., Zapata, L. A., Rodríguez, L. F., et al. 2013, MNRAS, 430, L10

Lombardi, M., Lada, C. J., \& Alves, J. 2008, A\&A, 480, 785

Looney, L. W., Mundy, L. G., \& Welch, W. J. 2000, ApJ, 529, 477

Lykke, J. M., Favre, C., Bergin, E. A., \& Jørgensen, J. K. 2015, A\&A, 582, A64

MacDonald, G. H., Gibb, A. G., Habing, R. J., \& Millar, T. J. 1996, A\&AS, 119, 333

Makiwa, G. 2014, Ph.D. Thesis, University of Lethbridge, Canada

Maret, S., Ceccarelli, C., Caux, E., et al. 2004, A\&A, 416, 577

Maret, S., Ceccarelli, C., Tielens, A. G. G. M., et al. 2005, A\&A, 442, 527

Mehringer, D. M., Snyder, L. E., Miao, Y., \& Lovas, F. J. 1997, ApJ, 480, L71

Mendoza, E., Lefloch, B., López-Sepulcre, A., et al. 2014, MNRAS, 445, 151

Menten, K. M., Serabyn, E., Guesten, R., \& Wilson, T. L. 1987, A\&A, 177, L57

Milam, S. N., Savage, C., Brewster, M. A., Ziurys, L. M., \& Wyckoff, S. 2005, ApJ, 634, 1126

Mizuno, A., Fukui, Y., Iwata, T., Nozawa, S., \& Takano, T. 1990, ApJ, 356, 184

Müller, H. S. P., \& Christen, D. 2004, J. Mol. Spectr., 228, 298

Müller, H. S. P., Thorwirth, S., Roth, D. A., \& Winnewisser, G. 2001, A\&A, 370, L49

Müller, H. S. P., Schlöder, F., Stutzki, J., \& Winnewisser, G. 2005, J. Mol. Struct., 742, 215

Müller, H. S. P., Belloche, A., Xu, L.-H., et al. 2016, A\&A, 587, A92

Mundy, L. G., Myers, S. T., \& Wilking, B. A. 1986, ApJ, 311, L75

Mundy, L. G., Wootten, H. A., \& Wilking, B. A. 1990, ApJ, 352, 159

Mundy, L. G., Wootten, A., Wilking, B. A., Blake, G. A., \& Sargent, A. I. 1992, ApJ, 385, 306

Murillo, N. M., Bruderer, S., van Dishoeck, E. F., et al. 2015, A\&A, 579, A114

Narayanan, G., Walker, C. K., \& Buckley, H. D. 1998, ApJ, 496, 292

Nisini, B., Giannini, T., \& Lorenzetti, D. 2002, ApJ, 574, 246

Nutter, D., Ward-Thompson, D., \& André, P. 2006, MNRAS, 368, 1833

Öberg, K. I., Bottinelli, S., Jørgensen, J. K., \& van Dishoeck, E. F. 2010, ApJ, 716,825

Ossenkopf, V., \& Henning, T. 1994, A\&A, 291, 943

Oya, Y., Sakai, N., Sakai, T., et al. 2014, ApJ, 795, 152

Oya, Y., Sakai, N., López-Sepulcre, A., et al. 2016, ApJ, 824, 88
Padgett, D. L., Rebull, L. M., Stapelfeldt, K. R., et al. 2008, ApJ, 672, 1013 Parise, B., Ceccarelli, C., Tielens, A. G. G. M., et al. 2002, A\&A, 393, L49 Parise, B., Castets, A., Herbst, E., et al. 2004, A\&A, 416, 159

Parise, B., Ceccarelli, C., Tielens, A. G. G. M., et al. 2006, A\&A, 453, 949

Parise, B., Du, F., Liu, F.-C., et al. 2012, A\&A, 542, L5

Pech, G., Loinard, L., Chandler, C. J., et al. 2010, ApJ, 712, 1403

Persson, M. V., Jørgensen, J. K., \& van Dishoeck, E. F. 2013, A\&A, 549, L3

Persson, M. V., Jørgensen, J. K., van Dishoeck, E. F., \& Harsono, D. 2014, A\&A, 563, A74

Pickett, H. M., Poynter, I. R. L., Cohen, E. A., et al. 1998, J. Quant. Spectr. Rad. Transf, 60, 883

Pineda, J. E., Maury, A. J., Fuller, G. A., et al. 2012, A\&A, 544, L7

Podio, L., Codella, C., Gueth, F., et al. 2015, A\&A, 581, A85

Rabli, D., \& Flower, D. R. 2010, MNRAS, 406, 95

Remijan, A., Snyder, L. E., Liu, S.-Y., Mehringer, D., \& Kuan, Y.-J. 2002, ApJ, 576, 264

Remijan, A. J., Markwick-Kemper, A., \& ALMA Working Group on Spectral Line Frequencies 2007, in Amer. Astron. Soc. Meet. Abstr., BAAS, 39, 963

Requena-Torres, M. A., Martín-Pintado, J., Martín, S., \& Morris, M. R. 2008 , ApJ, 672, 352

Richard, C., Margulès, L., Caux, E., et al. 2013, A\&A, 552, A117

Rivera, J. L., Loinard, L., Dzib, S. A., et al. 2015, ApJ, 807, 119

Roberts, H., \& Millar, T. J. 2000, A\&A, 361, 388

Roberts, H., Herbst, E., \& Millar, T. J. 2003, ApJ, 591, L41

Roueff, E., Lis, D. C., van der Tak, F. F. S., Gerin, M., \& Goldsmith, P. F. 2005, A\&A, 438, 585

Sakai, N., Sakai, T., \& Yamamoto, S. 2006, PASJ, 58, L15

Sakai, N., Sakai, T., Hirota, T., et al. 2014, Nature, 507, 78

Schöier, F. L., Jørgensen, J. K., van Dishoeck, E. F., \& Blake, G. A. 2002, A\&A, 390, 1001

Schöier, F. L., Jørgensen, J. K., van Dishoeck, E. F., \& Blake, G. A. 2004, A\&A, 418,185

Schöier, F. L., van der Tak, F. F. S., van Dishoeck, E. F., \& Black, J. H. 2005, A\&A, 432, 369

Senent, M. L. 2004, J. Phys. Chem. A, 108, 6286

Shiao, Y.-S. J., Looney, L. W., Remijan, A. J., Snyder, L. E., \& Friedel, D. N. 2010, ApJ, 716, 286

Shu, F. H., Adams, F. C., \& Lizano, S. 1987, ARA\&A, 25, 23

Smith, R. L., Pontoppidan, K. M., Young, E. D., \& Morris, M. R. 2015, ApJ, 813,120

Stark, R., van der Tak, F. F. S., \& van Dishoeck, E. F. 1999, ApJ, 521, L67

Stark, R., Sandell, G., Beck, S. C., et al. 2004, ApJ, 608, 341

Sugimura, M., Yamaguchi, T., Sakai, T., et al. 2011, PASJ, 63, 459

Tachihara, K., Mizuno, A., \& Fukui, Y. 2000, ApJ, 528, 817

Taquet, V., López-Sepulcre, A., Ceccarelli, C., et al. 2013, ApJ, 768, L29

Taquet, V., Charnley, S. B., \& Sipilä, O. 2014, ApJ, 791, 1

Taquet, V., López-Sepulcre, A., Ceccarelli, C., et al. 2015, ApJ, 804, 81

Terebey, S., Shu, F. H., \& Cassen, P. 1984, ApJ, 286, 529

van der Tak, F. F. S., van Dishoeck, E. F., Evans, N. J., \& Blake, G. A. 2000, ApJ, 537, 283

van der Tak, F. F. S., Black, J. H., Schöier, F. L., Jansen, D. J., \& van Dishoeck, E. F. 2007, A\&A, 468, 627

van Dishoeck, E. F., Blake, G. A., Draine, B. T., \& Lunine, J. I. 1993, in Protostars and Planets III, eds. E. H. Levy, \& J. I. Lunine, 163

van Dishoeck, E. F., Blake, G. A., Jansen, D. J., \& Groesbeck, T. D. 1995, ApJ, 447, 760

Vastel, C., Phillips, T. G., \& Yoshida, H. 2004, ApJ, 606, L127

Vastel, C., Ceccarelli, C., Caux, E., et al. 2010, A\&A, 521, L31

Vastel, C., Ceccarelli, C., Lefloch, B., \& Bachiller, R. 2014, ApJ, 795, L2

Walker, C. K., Lada, C. J., Young, E. T., Maloney, P. R., \& Wilking, B. A. 1986, ApJ, 309, L47

Walker, C. K., Lada, C. J., Young, E. T., \& Margulis, M. 1988, ApJ, 332, 335

Walker, C. K., Adams, F. C., \& Lada, C. J. 1990, ApJ, 349, 515

Whittet, D. C. B., \& van Breda, I. G. 1975, Ap\&SS, 38, L3

Widicus Weaver, S. L., Butler, R. A. H., Drouin, B. J., et al. 2005, ApJS, 158, 188

Wilson, T. L., \& Rood, R. 1994, ARA\&A, 32, 191

Wootten, A. 1989, ApJ, 337, 858

Wootten, A., \& Loren, R. B. 1987, ApJ, 317, 220

Yeh, S. C. C., Hirano, N., Bourke, T. L., et al. 2008, ApJ, 675, 454

Young, K. E., Enoch, M. L., Evans, II, N. J., et al. 2006, ApJ, 644, 326

Zapata, L. A., Loinard, L., Rodríguez, L. F., et al. 2013, ApJ, 764, L14

Zhou, S. 1995, ApJ, 442, 685 
J. K. Jørgensen et al.: The ALMA Protostellar Interferometric Line Survey (PILS)

\section{Appendix A: Fitted lines of glycolaldehyde and ethylene glycol in Band 3 data}

Table A.1. Parameters for identified glycolaldehyde and ethylene glycol transitions measured in the Band 3 (3 mm) dataset.

\begin{tabular}{|c|c|c|c|c|c|c|c|}
\hline Transition & $\begin{array}{l}\text { Freq } \\
{[\mathrm{GHz}]}\end{array}$ & $\begin{array}{l}E_{\mathrm{up}} \\
{[\mathrm{K}]}\end{array}$ & $\begin{array}{l}A_{\mathrm{ul}} \\
{\left[\mathrm{s}^{-1}\right]}\end{array}$ & $g_{\mathrm{u}}$ & $\begin{array}{l}I \\
{\left[\mathrm{Jy} \mathrm{beam}^{-1}\right]}\end{array}$ & $\begin{array}{l}V_{\mathrm{lsr}} \\
{\left[\mathrm{km} \mathrm{s}^{-1}\right]}\end{array}$ & $\begin{array}{l}\delta v \\
{\left[\mathrm{~km} \mathrm{~s}^{-1}\right]}\end{array}$ \\
\hline \multicolumn{8}{|c|}{ Glycolaldehyde } \\
\hline $20_{4,16}-20_{3,17}$ & 89.6164 & 130.4 & $2.00 \times 10^{-5}$ & 41 & 0.062 & 2.6 & 1.5 \\
\hline $10_{4,7}-10_{3,8}$ & 89.6441 & 40.3 & $1.59 \times 10^{-5}$ & 21 & 0.057 & 2.5 & 1.6 \\
\hline $8_{4,5}-8_{3,6}$ & 89.7022 & 404.1 & $1.47 \times 10^{-5}$ & 17 & 0.013 & 2.8 & 1.8 \\
\hline $9,9-8,8$ & 92.8515 & 304.0 & $2.67 \times 10^{-5}$ & 19 & 0.022 & 2.6 & 1.7 \\
\hline $12_{4,9}-12_{3,10}$ & 92.8539 & 53.2 & $1.83 \times 10^{-5}$ & 25 & 0.061 & 2.5 & 1.0 \\
\hline $25_{6,19}-25_{5,20}$ & 92.8821 & 485.9 & $2.71 \times 10^{-5}$ & 51 & 0.023 & 2.5 & 1.7 \\
\hline $10_{1,9}-9_{2,8}$ & 102.5062 & 406.1 & $2.12 \times 10^{-5}$ & 21 & 0.013 & 2.8 & 1.3 \\
\hline $7_{2,6}-6_{1,5}$ & 102.5498 & 17.8 & $1.91 \times 10^{-5}$ & 15 & 0.055 & 2.7 & 1.2 \\
\hline $14_{3,12}-14_{2,13}$ & 102.5729 & 63.6 & $1.82 \times 10^{-5}$ & 29 & 0.064 & 2.6 & 1.3 \\
\hline $18_{3,15}-18_{2,16}$ & 102.6144 & 104.1 & $2.29 \times 10^{-5}$ & 37 & 0.073 & 2.6 & 1.2 \\
\hline $7_{2,6}-6_{1,5}$ & 102.6690 & 392.0 & $1.91 \times 10^{-5}$ & 15 & 0.011 & 2.8 & 1.2 \\
\hline $10_{0,10}-9_{1,9}$ & 103.3913 & 28.3 & $2.78 \times 10^{-5}$ & 21 & 0.086 & 2.7 & 1.2 \\
\hline \multicolumn{8}{|c|}{$a G g^{\prime}$ ethylene glycol } \\
\hline $8_{3,5} v=1-7_{3,4} v=0$ & 89.7082 & 22.5 & $1.45 \times 10^{-5}$ & 153 & 0.037 & 2.7 & 1.0 \\
\hline $36_{7,29} v=1-36_{6,30} v=1$ & 92.8604 & 357.3 & $3.53 \times 10^{-6}$ & 657 & 0.010 & 2.6 & 1.7 \\
\hline $9_{1,9} v=1-8_{1,8} v=0$ & 92.9759 & 21.6 & $1.80 \times 10^{-5}$ & 171 & 0.049 & 2.7 & 1.1 \\
\hline $41_{8,33} v=0-41_{7,34} v=0$ & 102.5189 & 461.2 & $4.51 \times 10^{-6}$ & 747 & 0.013 & 2.9 & 1.1 \\
\hline $9_{2,7} v=1-8_{2,6} v=0$ & 102.5394 & 25.0 & $2.38 \times 10^{-5}$ & 133 & 0.048 & 2.6 & 0.90 \\
\hline $10_{0,10} v=1-9_{0,9} v=0$ & 102.6898 & 26.1 & $2.63 \times 10^{-5}$ & 189 & 0.068 & 2.4 & 1.5 \\
\hline \multicolumn{8}{|c|}{$g G g^{\prime}$ ethylene glycol } \\
\hline $9_{6,4} v=1-8_{6,3} v=0$ & 92.8017 & 39.5 & $3.95 \times 10^{-6}$ & 171 & 0.018 & 2.5 & 1.3 \\
\hline $9_{6,3} v=1-8_{6,2} v=0^{a}$ & 92.8017 & $\ldots$ & $\ldots$ & 133 & $\ldots$ & $\ldots$ & $\ldots$ \\
\hline $9_{5,5} v=1-8_{5,4} v=0^{b}$ & 92.9120 & 34.2 & $4.94 \times 10^{-6}$ & 171 & $\ldots$ & $\ldots$ & $\ldots$ \\
\hline $9_{5,4} v=1-8_{5,3} v=0$ & 92.9144 & 34.2 & $4.94 \times 10^{-6}$ & 133 & 0.011 & 2.7 & 0.87 \\
\hline $22_{6,17} v=1-22_{5,17} v=0$ & 92.9184 & 141.6 & $4.12 \times 10^{-6}$ & 315 & 0.011 & 2.7 & 0.93 \\
\hline $10_{4,7} v=1-9_{4,6} v=0$ & 103.3723 & 34.7 & $8.30 \times 10^{-6}$ & 147 & 0.014 & 2.4 & 1.3 \\
\hline
\end{tabular}

Notes. ${ }^{(a)}$ Blended with above transition. ${ }^{(b)}$ Complete overlap with prominent acetaldehyde $\left(\mathrm{CH}_{3} \mathrm{CHO}\right)$ line; excluded from fit. 
Appendix B: Synthetic spectra fits for glycolaldehyde, ethylene glycol, and acetic acid
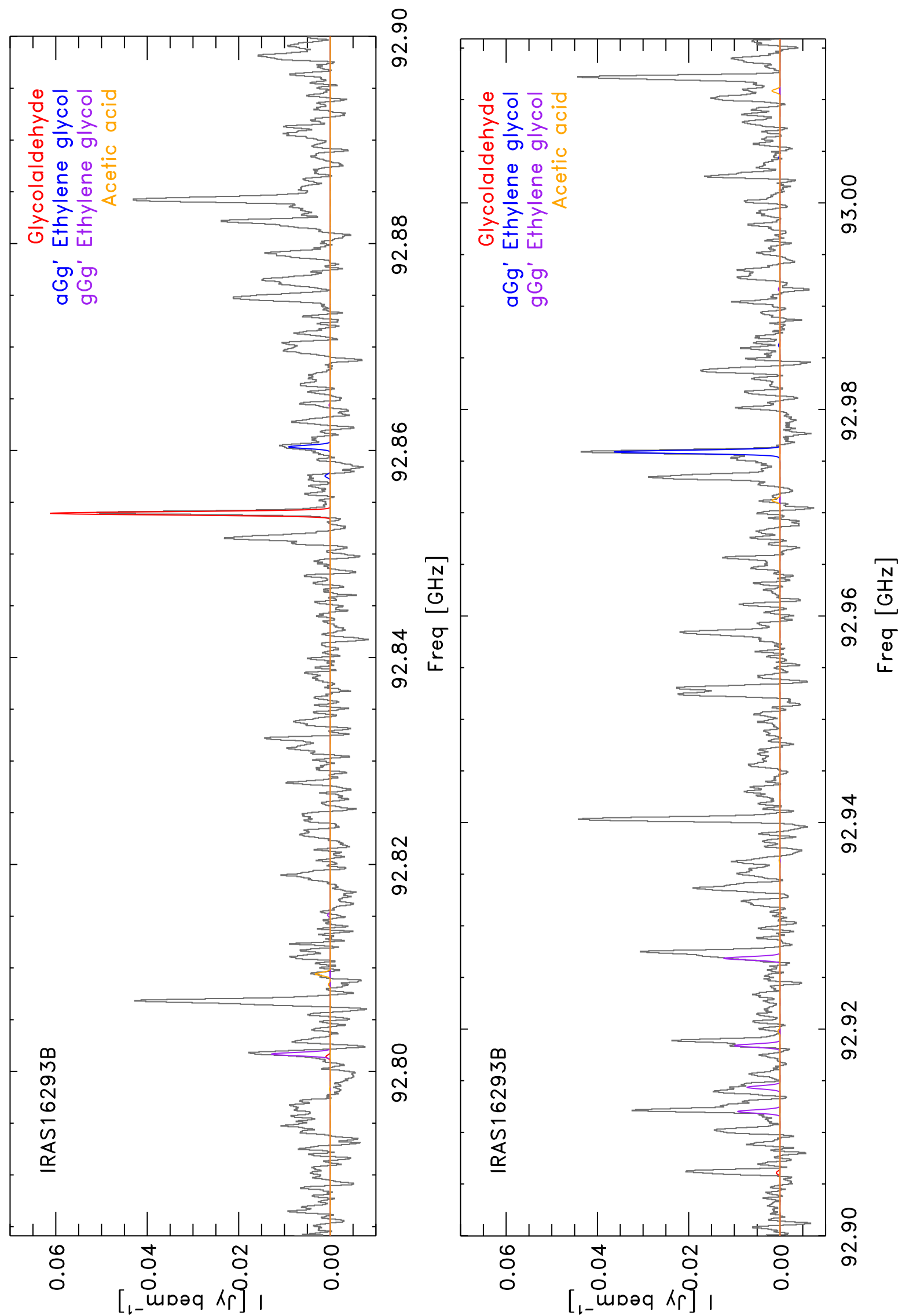

Fig. B.1. Fits to the glycolaldehyde, ethylene glycol, and acetic acid lines at $3 \mathrm{~mm}$ (Band 3). 
J. K. Jørgensen et al.: The ALMA Protostellar Interferometric Line Survey (PILS)

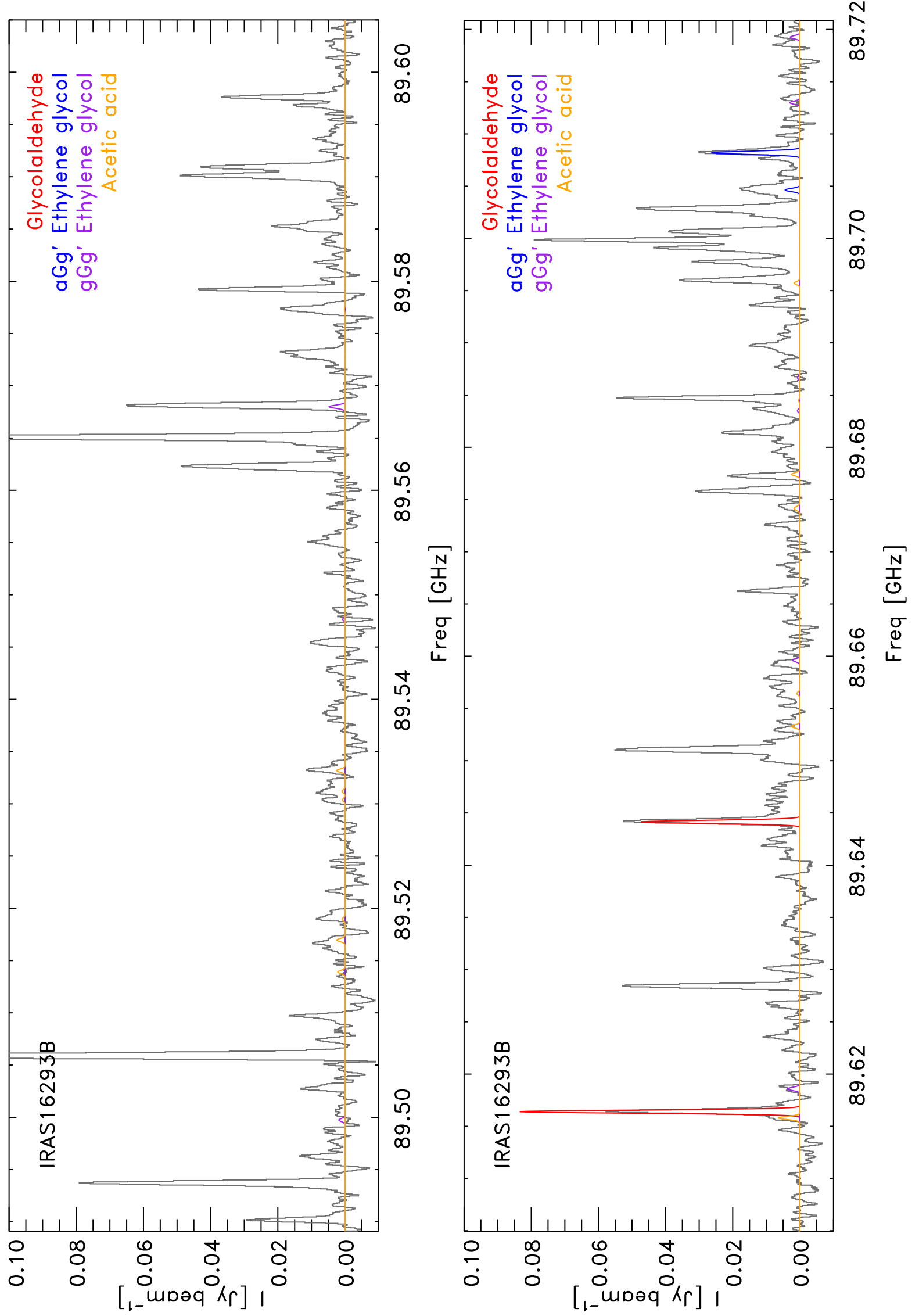

Fig. B.1. continued. 
A\&A 595, A117 (2016)

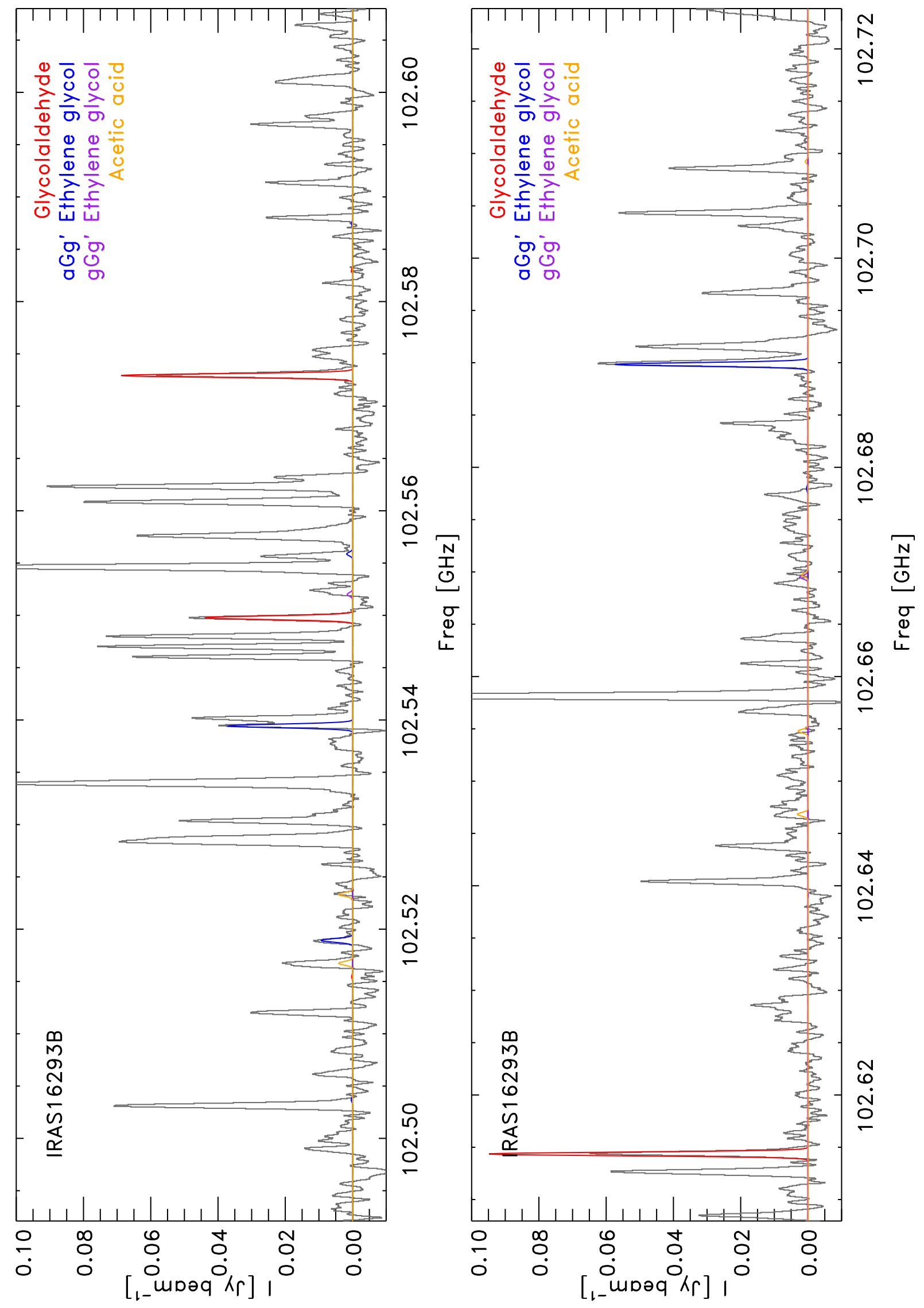

Fig. B.1. continued. 
J. K. Jørgensen et al.: The ALMA Protostellar Interferometric Line Survey (PILS)

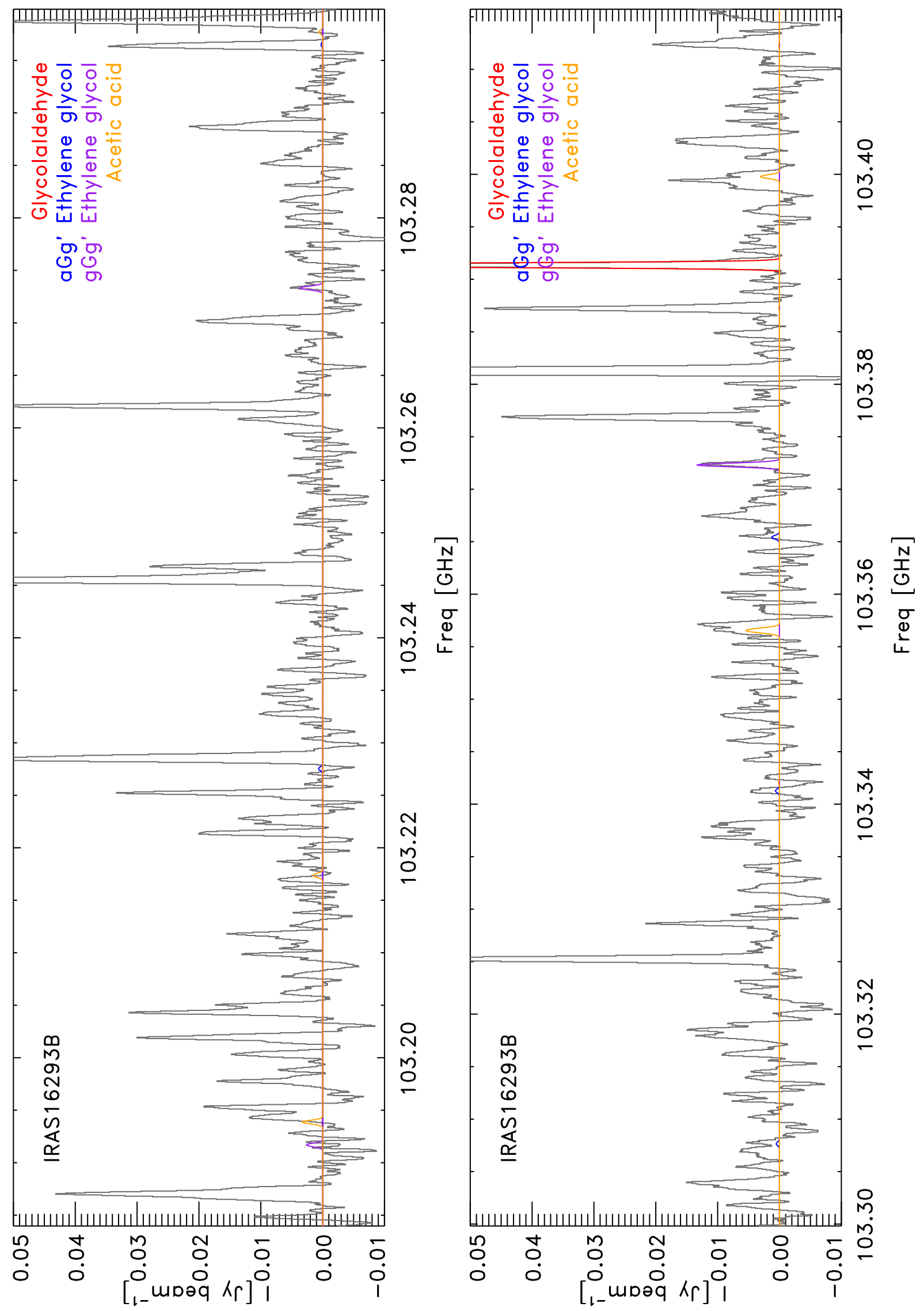

Fig. B.1. continued. 
A\&A 595, A117 (2016)
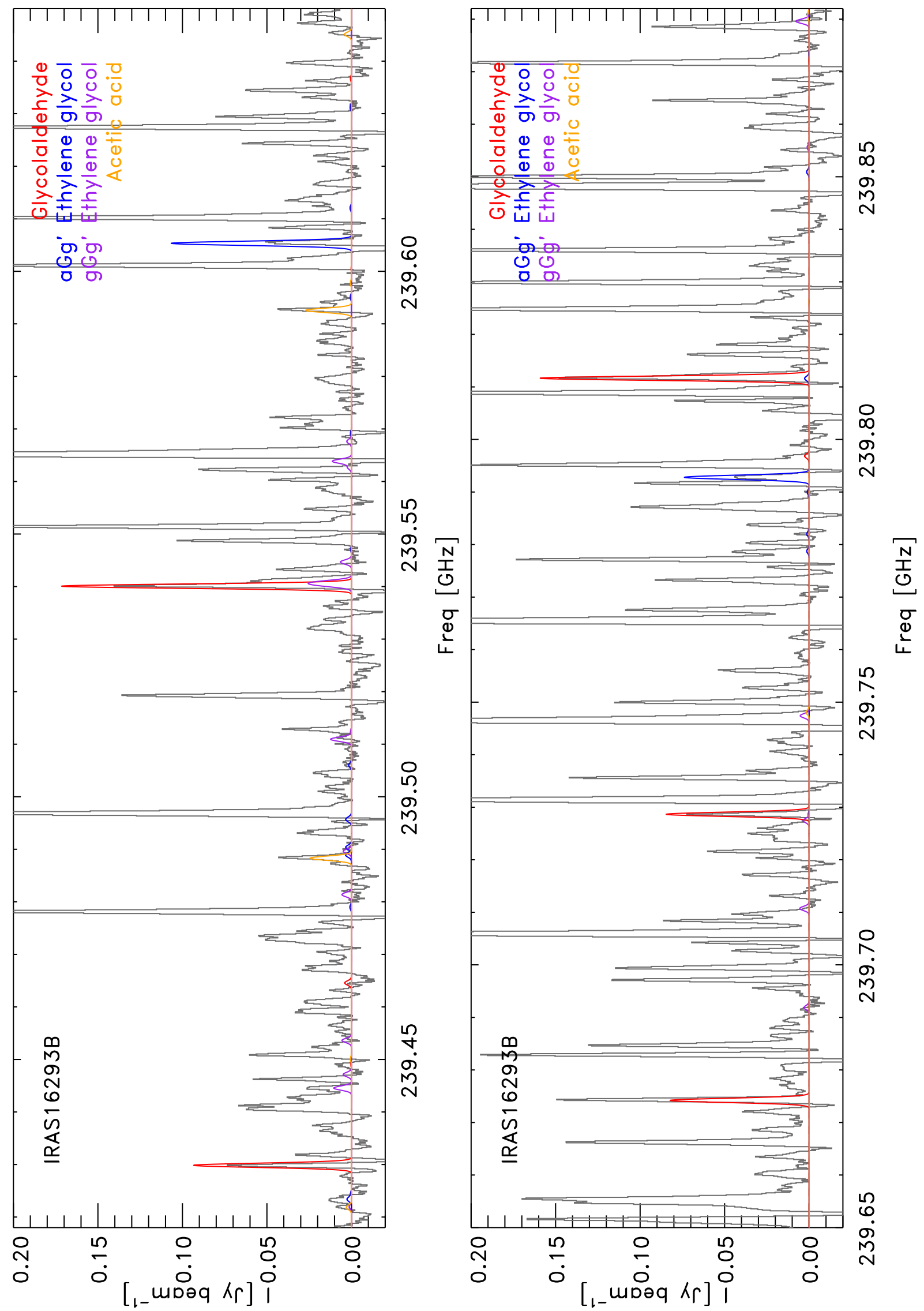

Fig. B.2. Fits to the glycolaldehyde, ethylene glycol, and acetic acid lines at $1.3 \mathrm{~mm}$ (Band 6). 
J. K. Jørgensen et al.: The ALMA Protostellar Interferometric Line Survey (PILS)

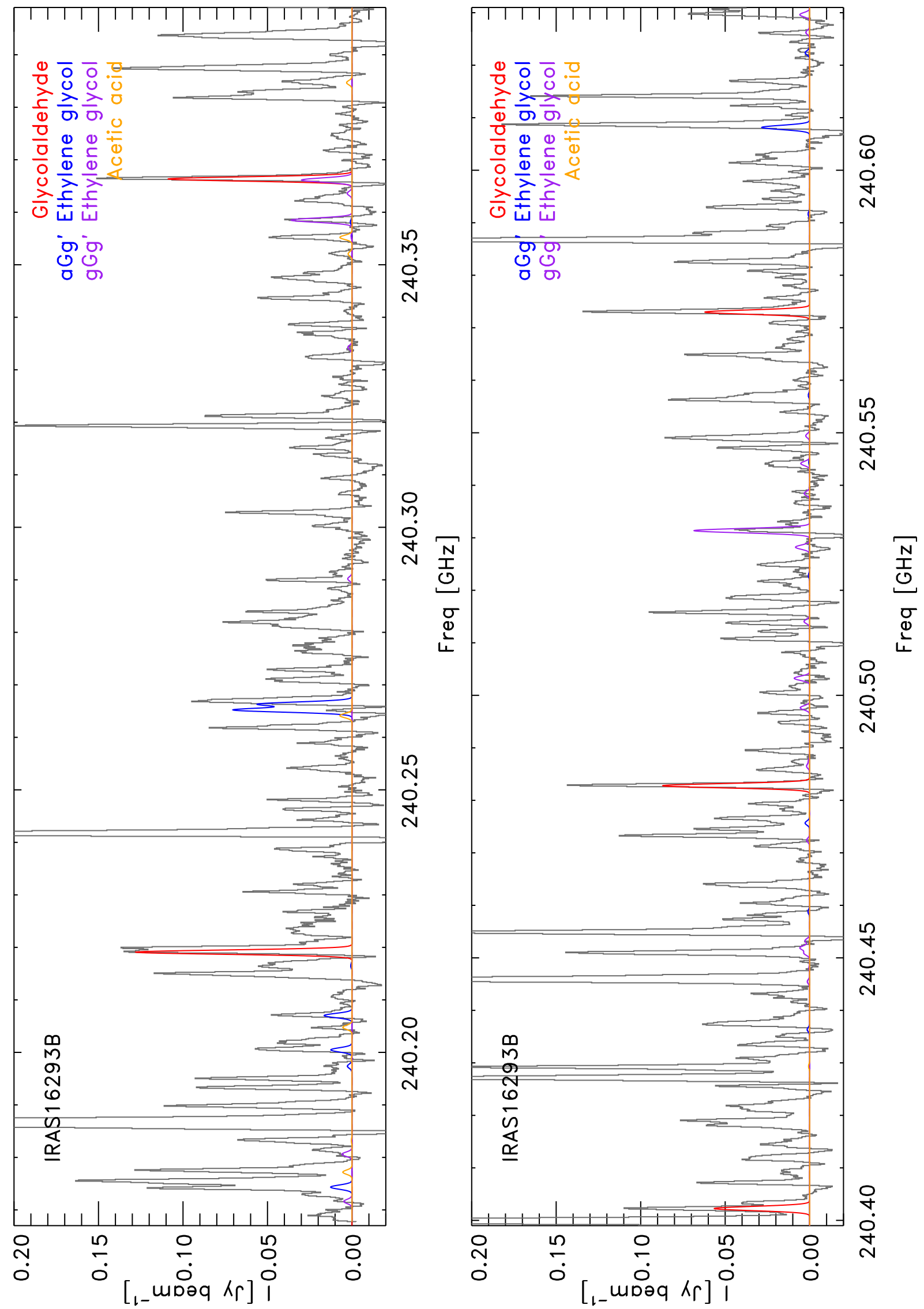

Fig. B.2. continued. 
A\&A 595, A117 (2016)
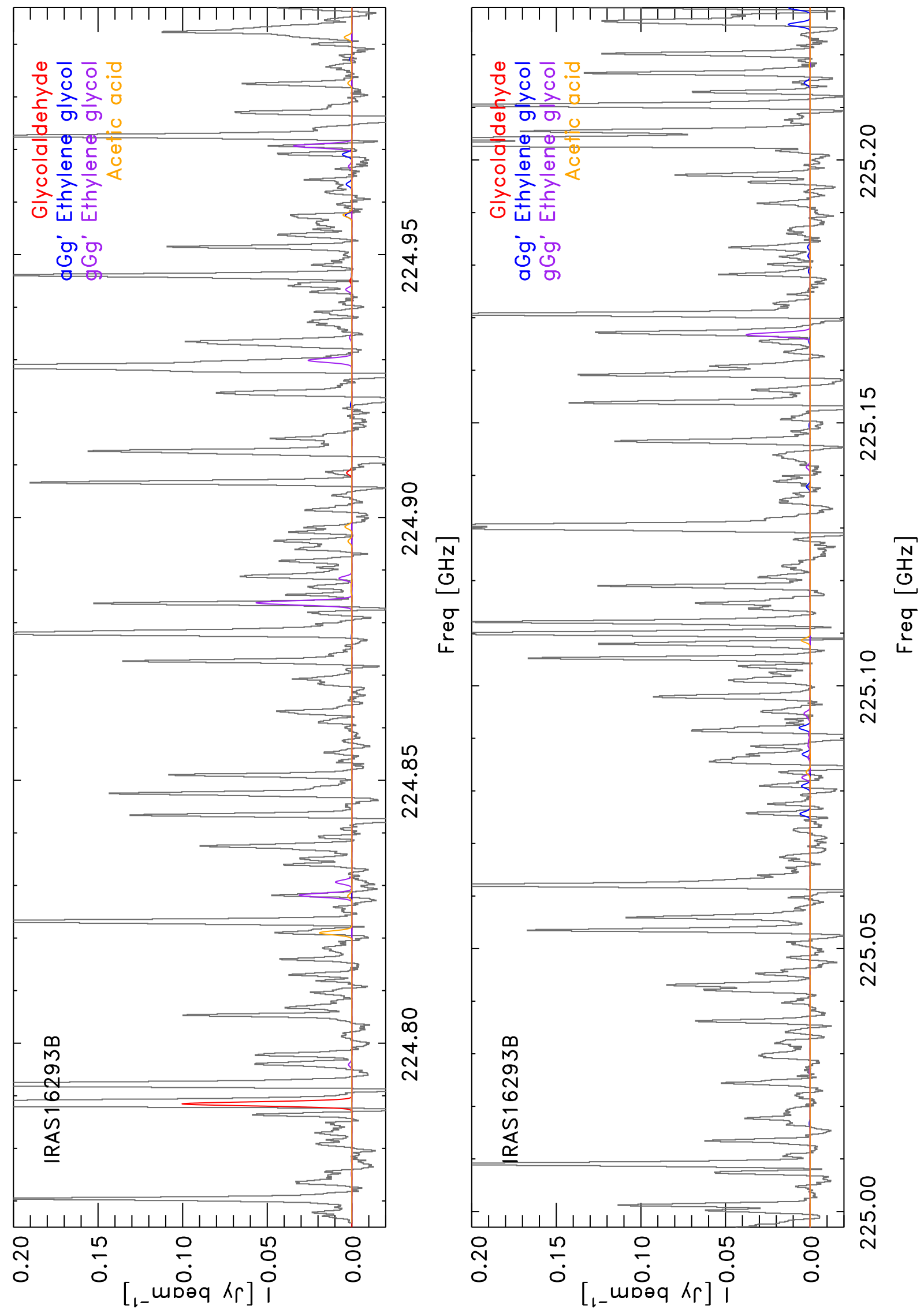

Fig. B.2. continued. 
J. K. Jørgensen et al.: The ALMA Protostellar Interferometric Line Survey (PILS)

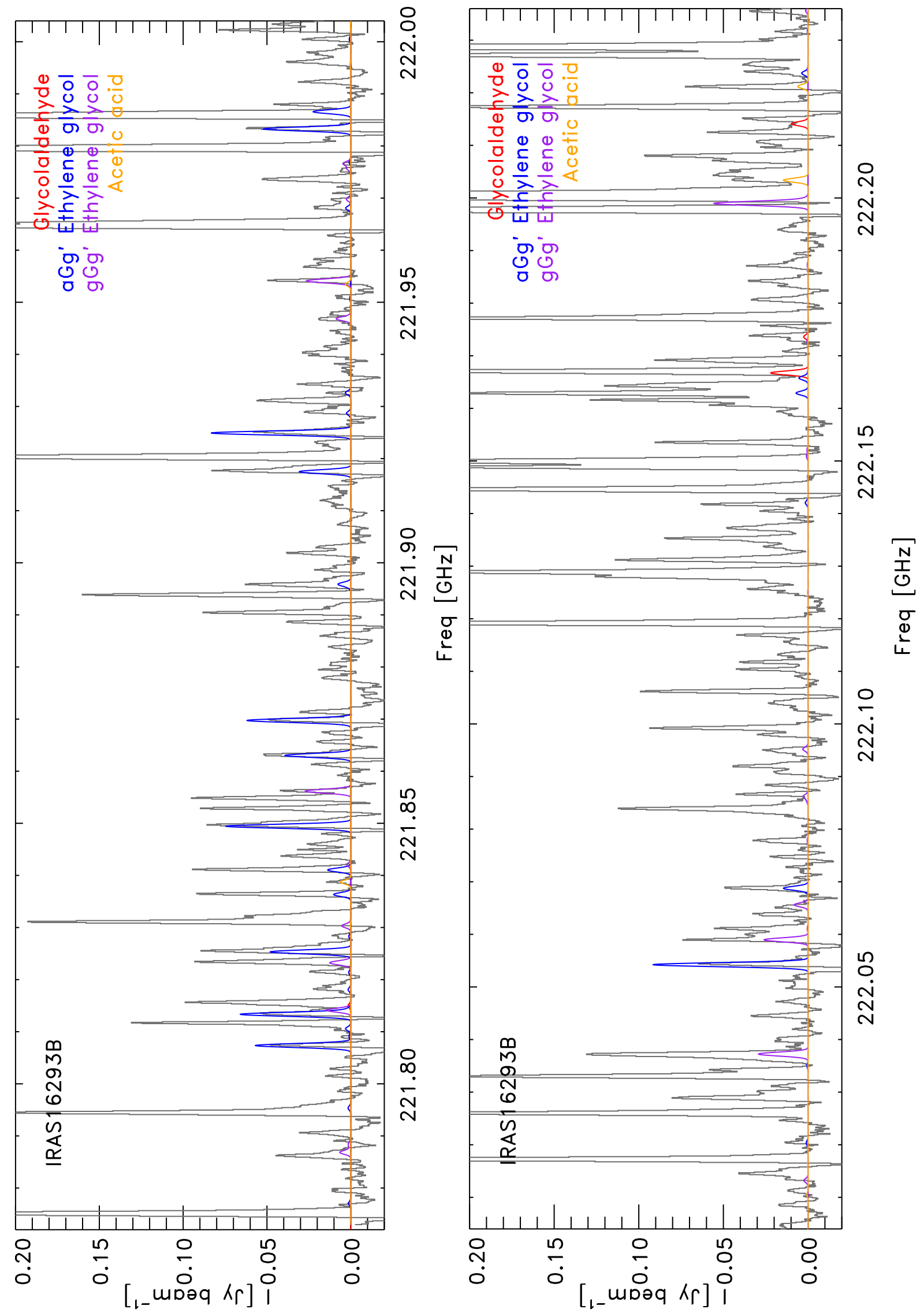

Fig. B.2. continued. 
A\&A 595, A117 (2016)
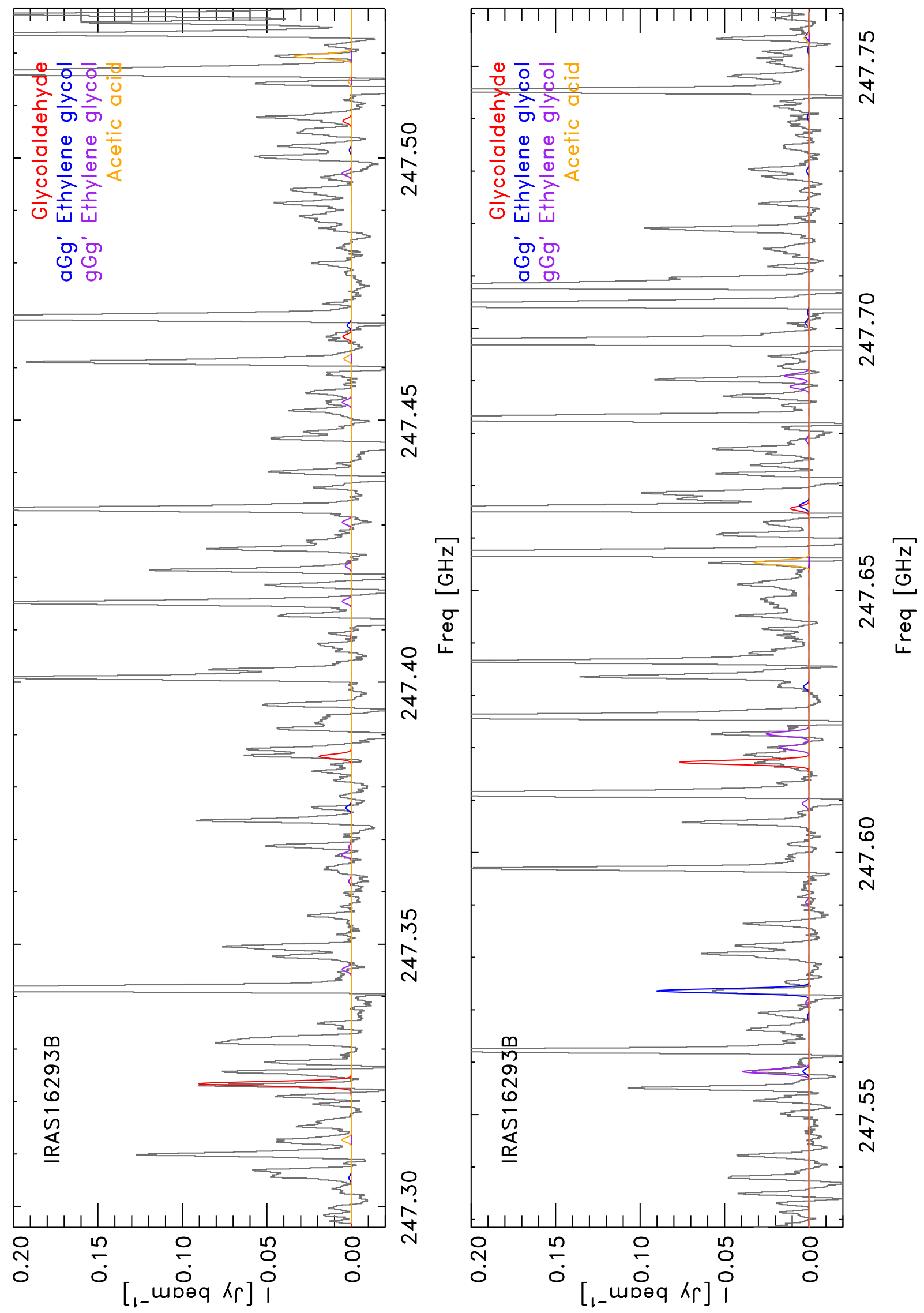

Fig. B.2. continued. 
J. K. Jørgensen et al.: The ALMA Protostellar Interferometric Line Survey (PILS)

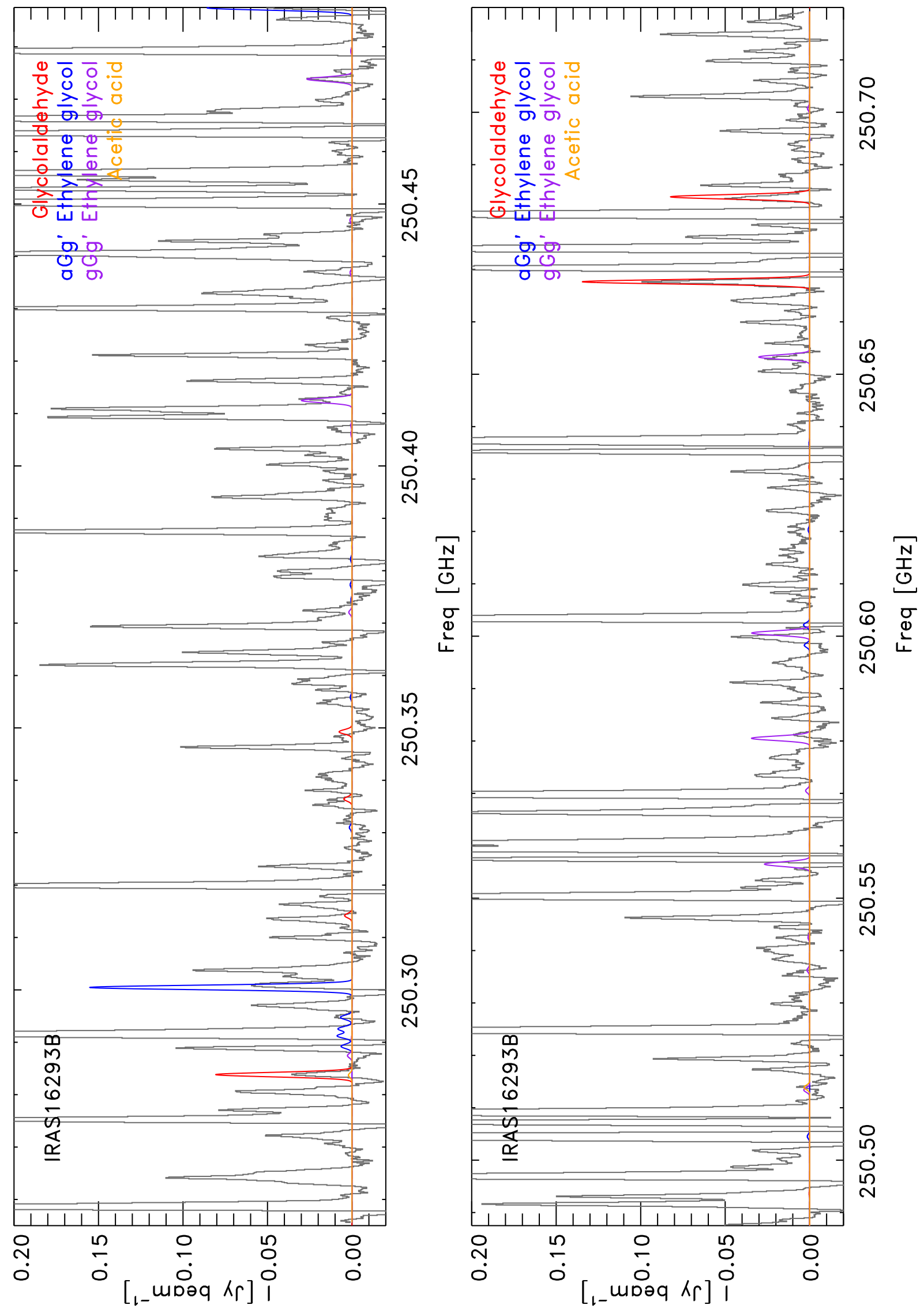

Fig. B.2. continued. 
A\&A 595, A117 (2016)

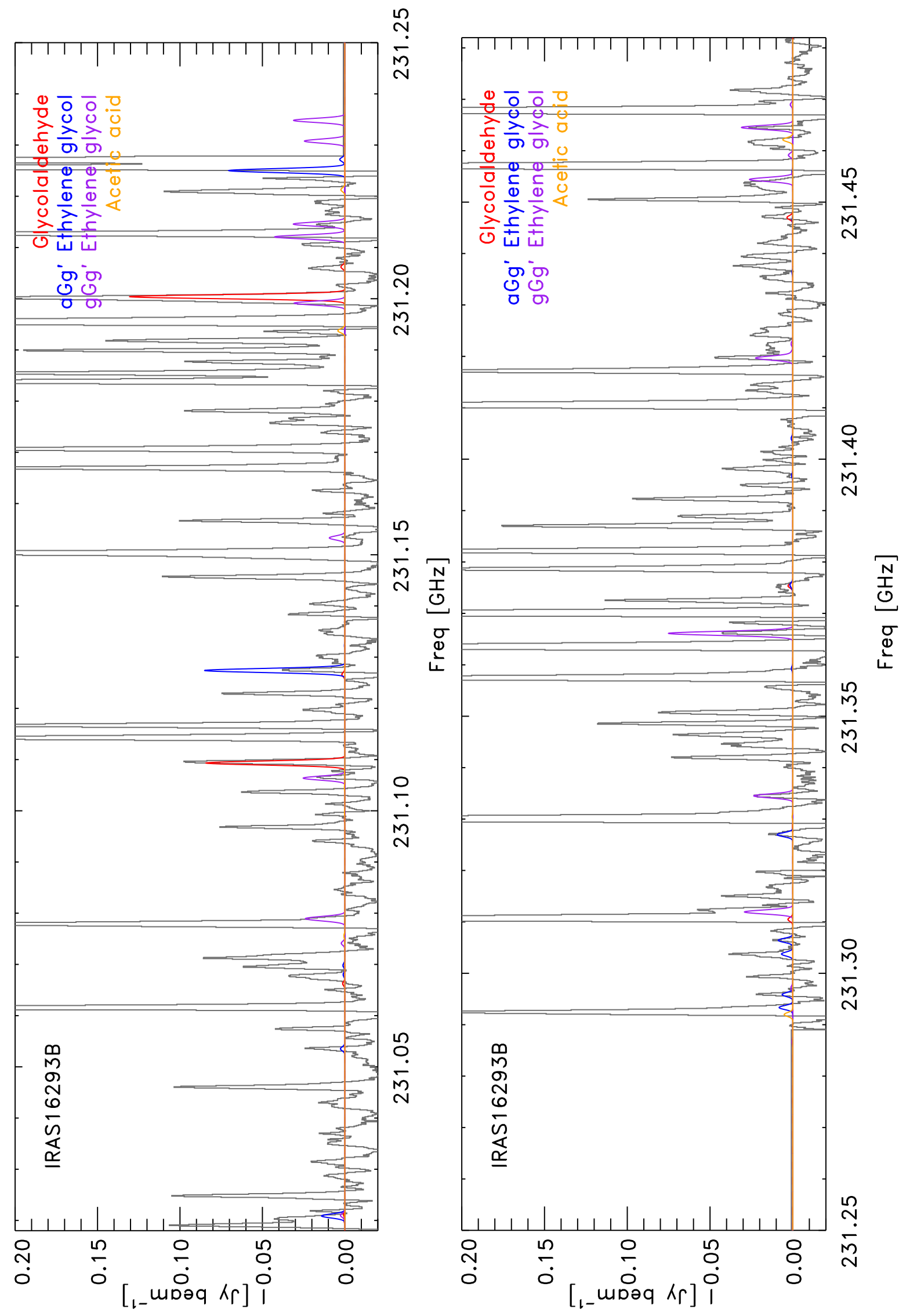

Fig. B.2. continued. 
J. K. Jørgensen et al.: The ALMA Protostellar Interferometric Line Survey (PILS)

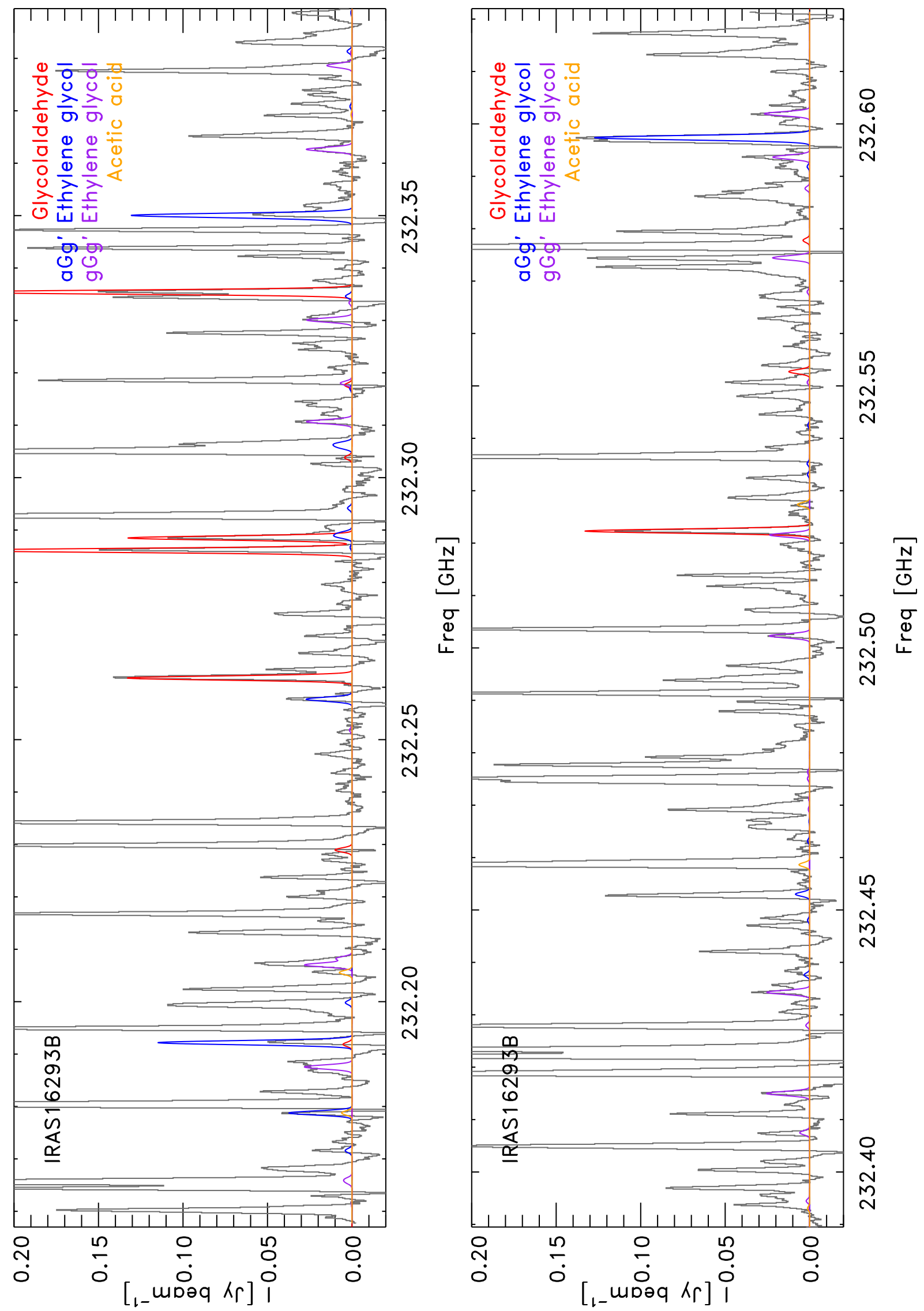

Fig. B.2. continued. 


\section{Appendix C: Synthetic spectra fits for species in Band 7}

C.1. Methanol $\left(\mathrm{CH}_{3}^{18} \mathrm{OH}\right)$
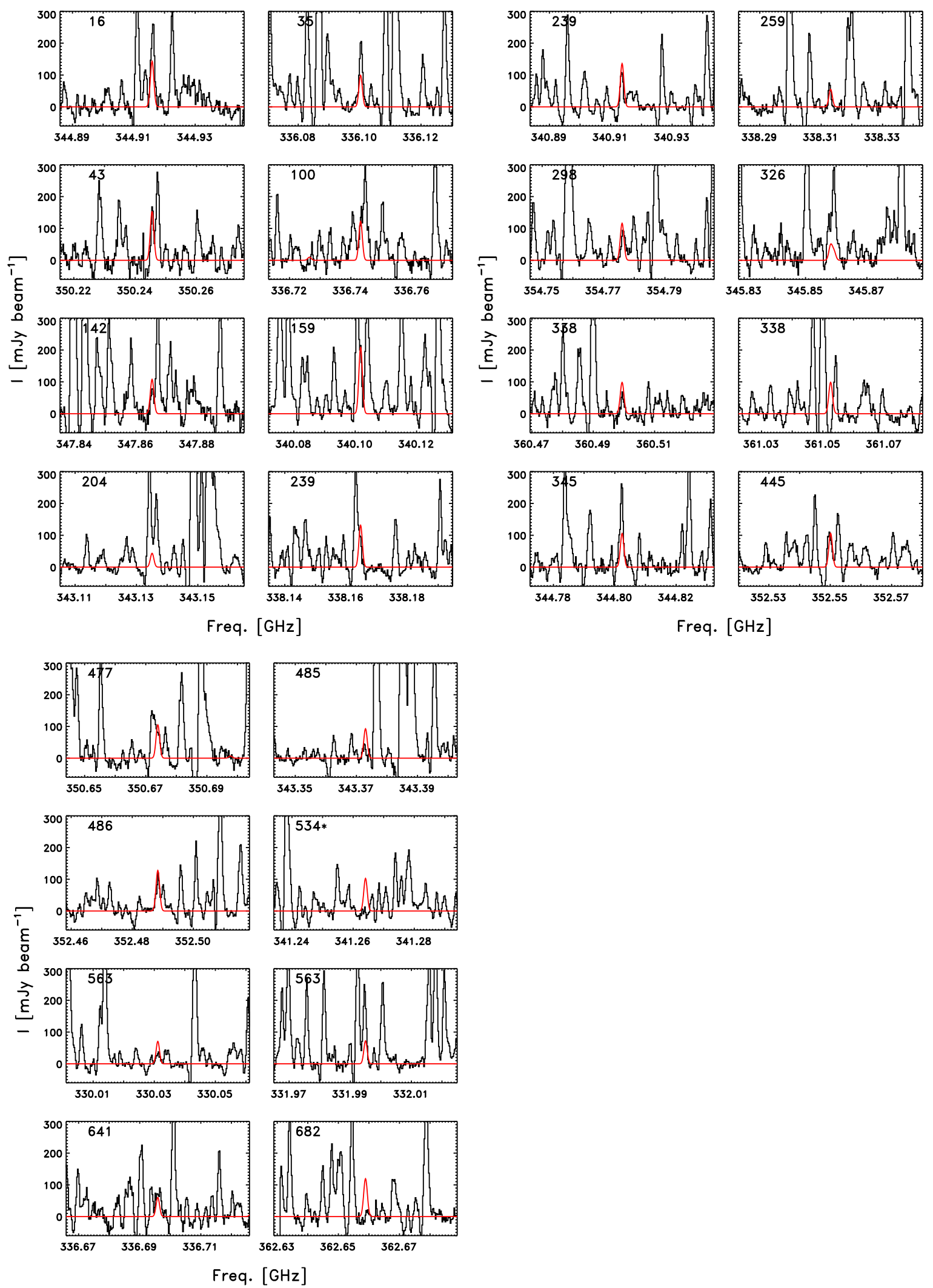

Fig. C.1. The 24 brightest lines of $\mathrm{CH}_{3}^{18} \mathrm{OH}$ as expected from the synthetic spectrum. These lines are sorted according to $E_{\text {up }}$ given in $\mathrm{K}$ in the upper left corner of each panel. For frequencies where multiple transitions are overlapping an asterisk (*) is added after the value of $E_{\text {up}}$. 
C.2. Glycolaldehyde
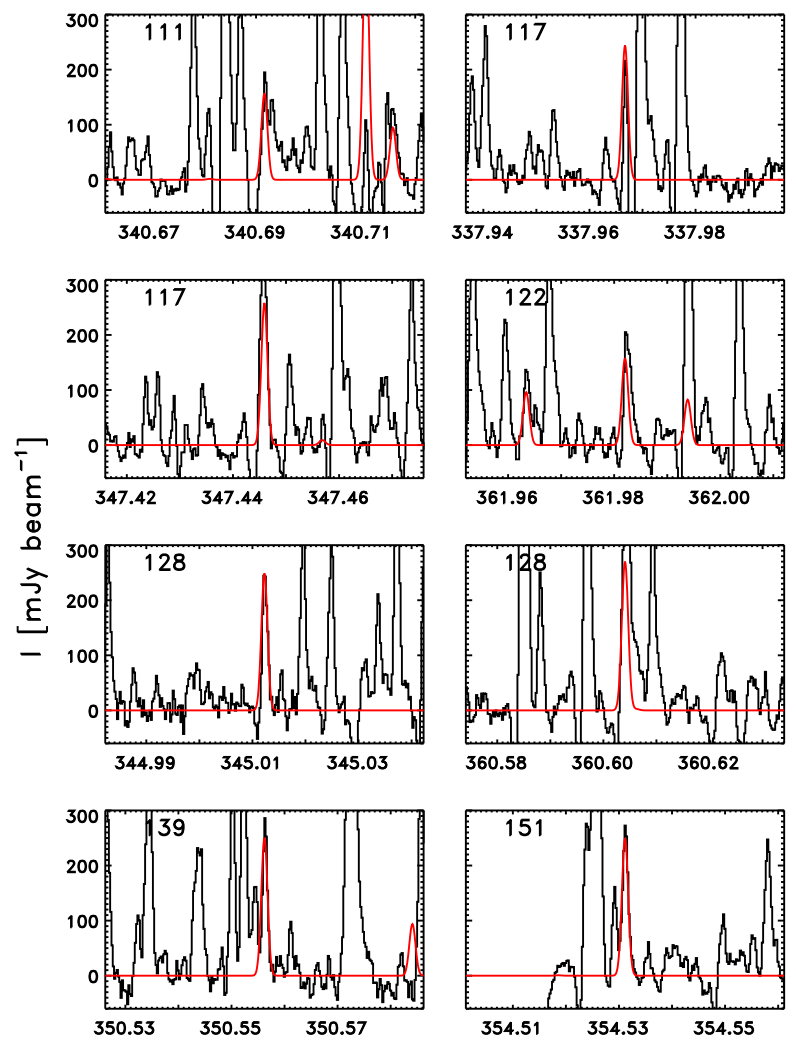

Freq. [GHz]
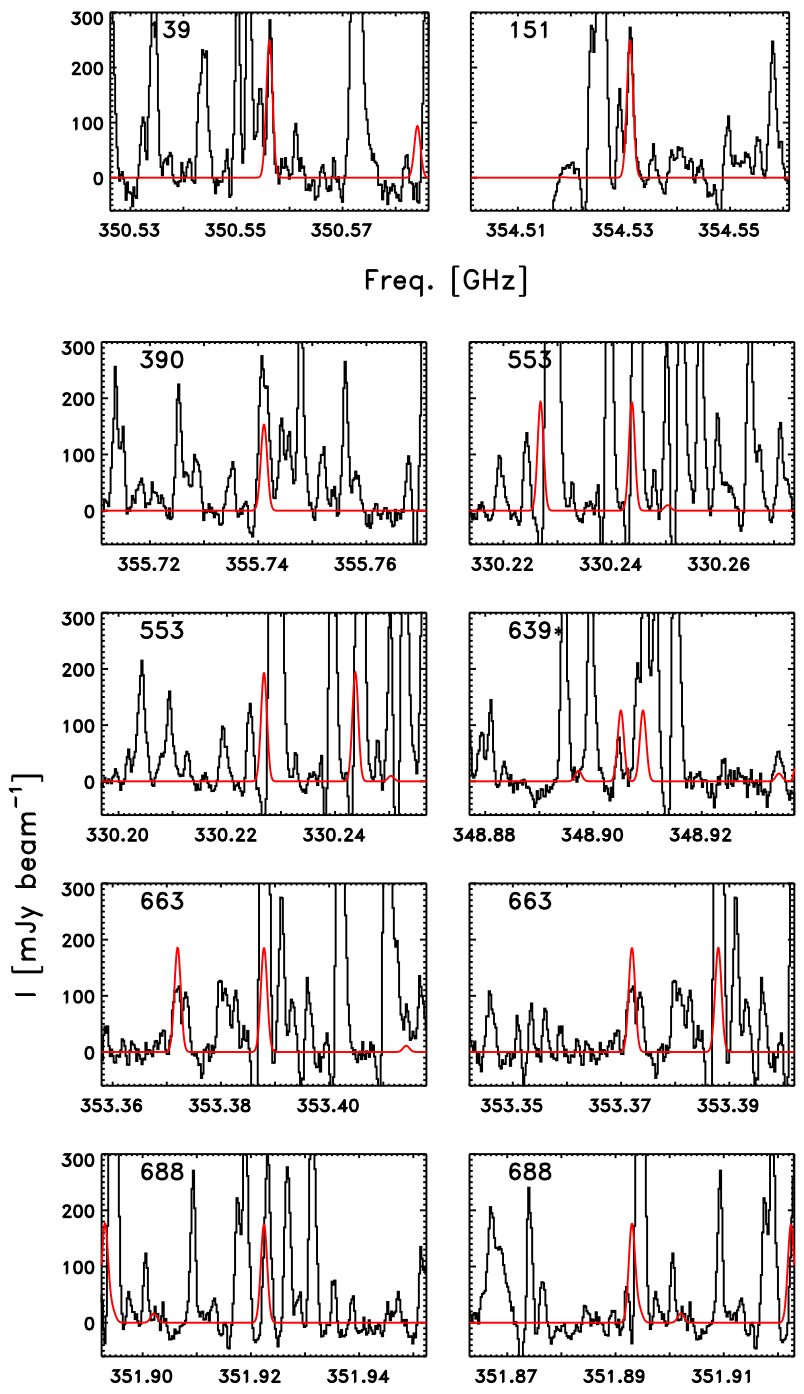
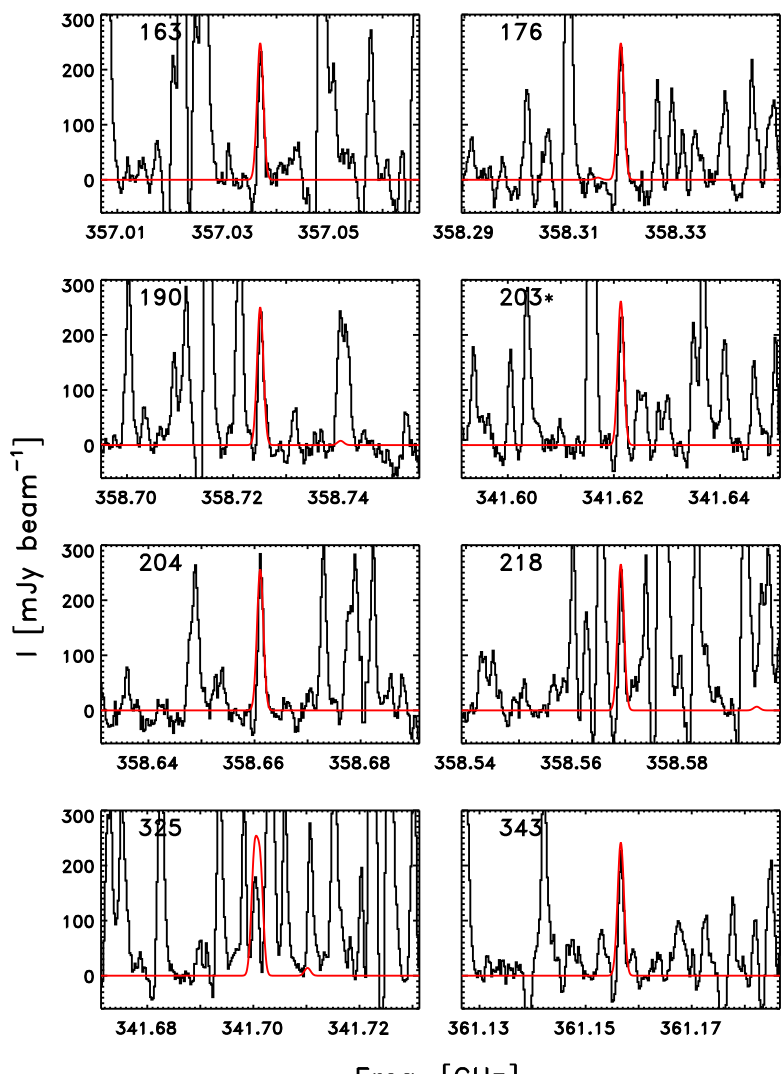

Freq. [GHz]

Freq. [GHz]

Fig. C.2. As in Fig. C.1 for the 24 brightest lines of glycolaldehyde with $\tau<0.2$ as expected from the synthetic spectrum. 
C.3. $a G g^{\prime}$ ethylene glycol
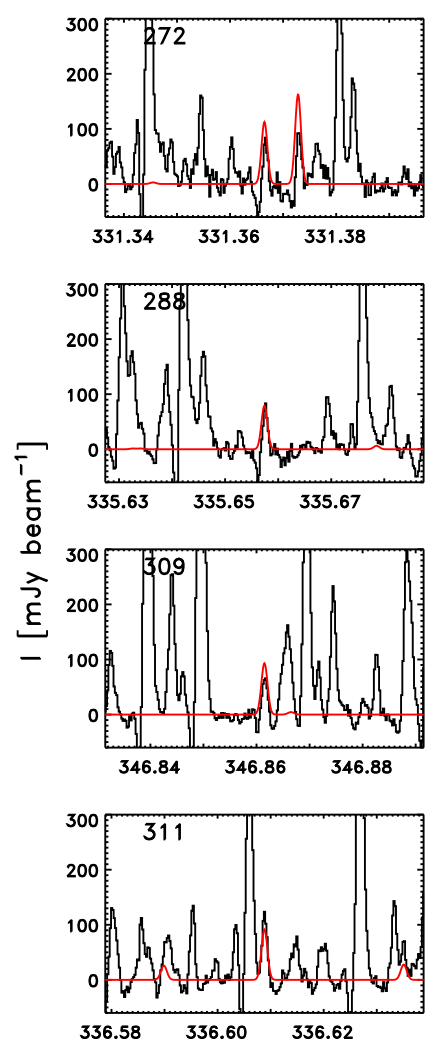

Freq. $[\mathrm{GHz}]$
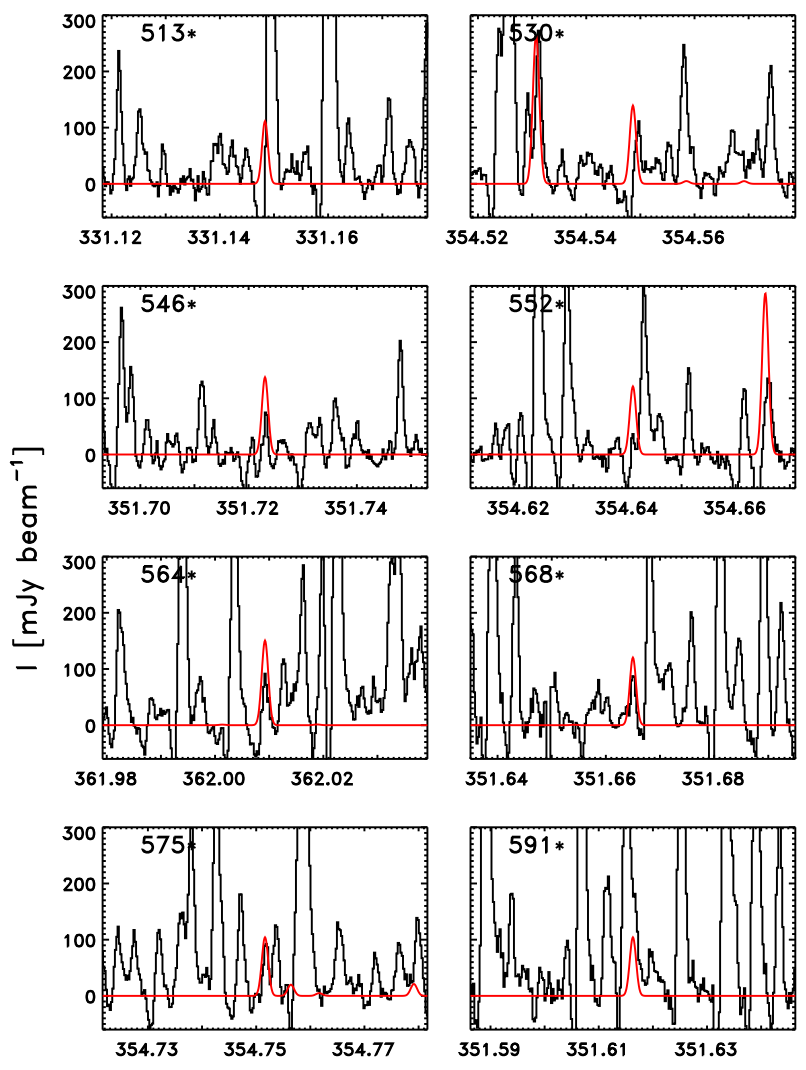

Freq. [GHz]
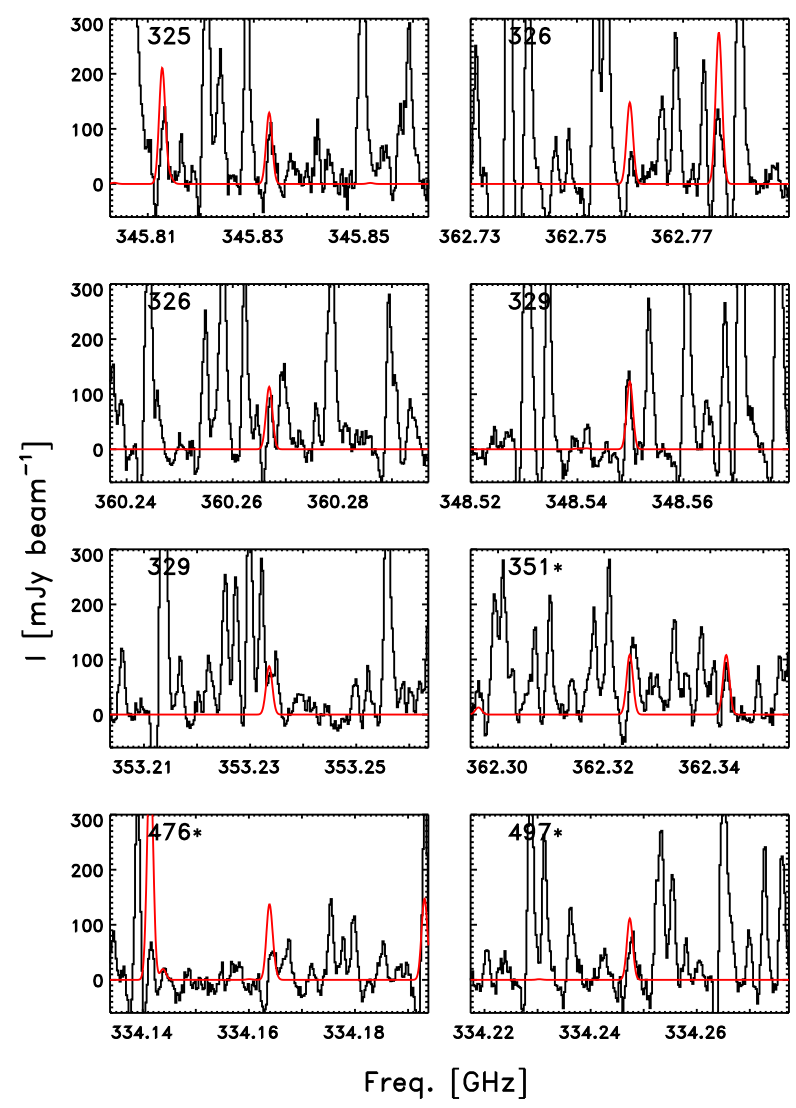

Fig. C.3. As in Fig. C.1 for the 24 brightest lines of $a G g^{\prime}$ ethylene glycol with $\tau<0.2$ as expected from the synthetic spectrum. 
C.4. $g G g^{\prime}$ ethylene glycol
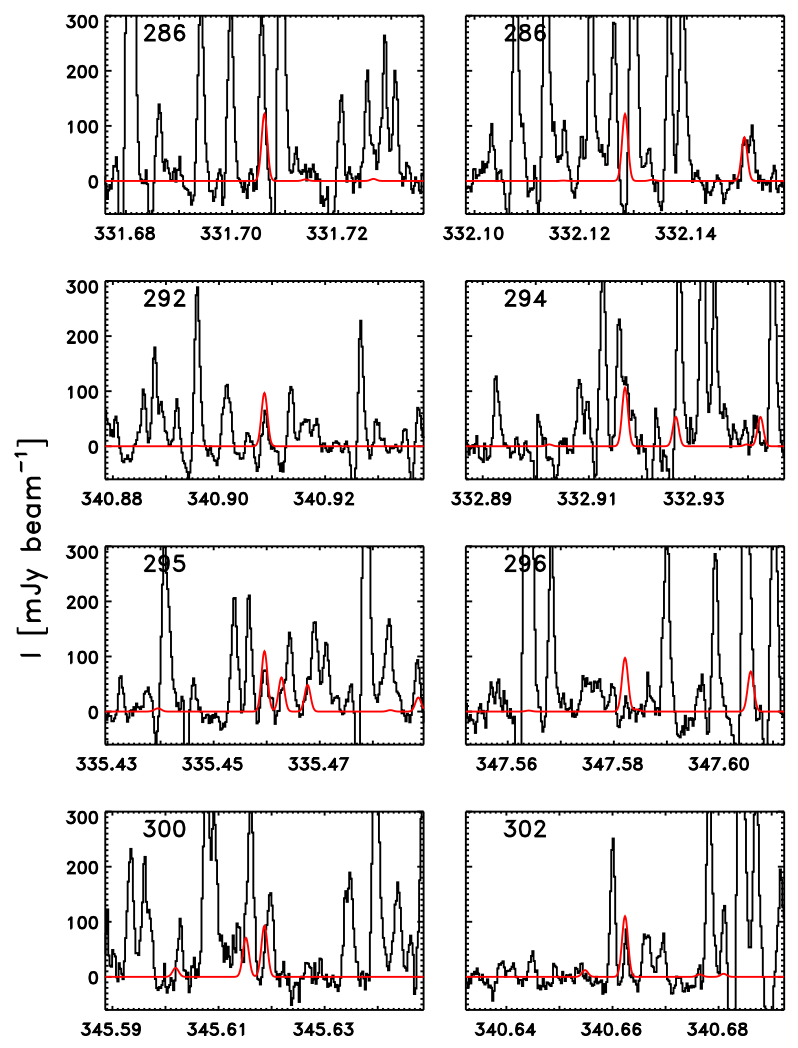

Freq. $[\mathrm{GHz}]$
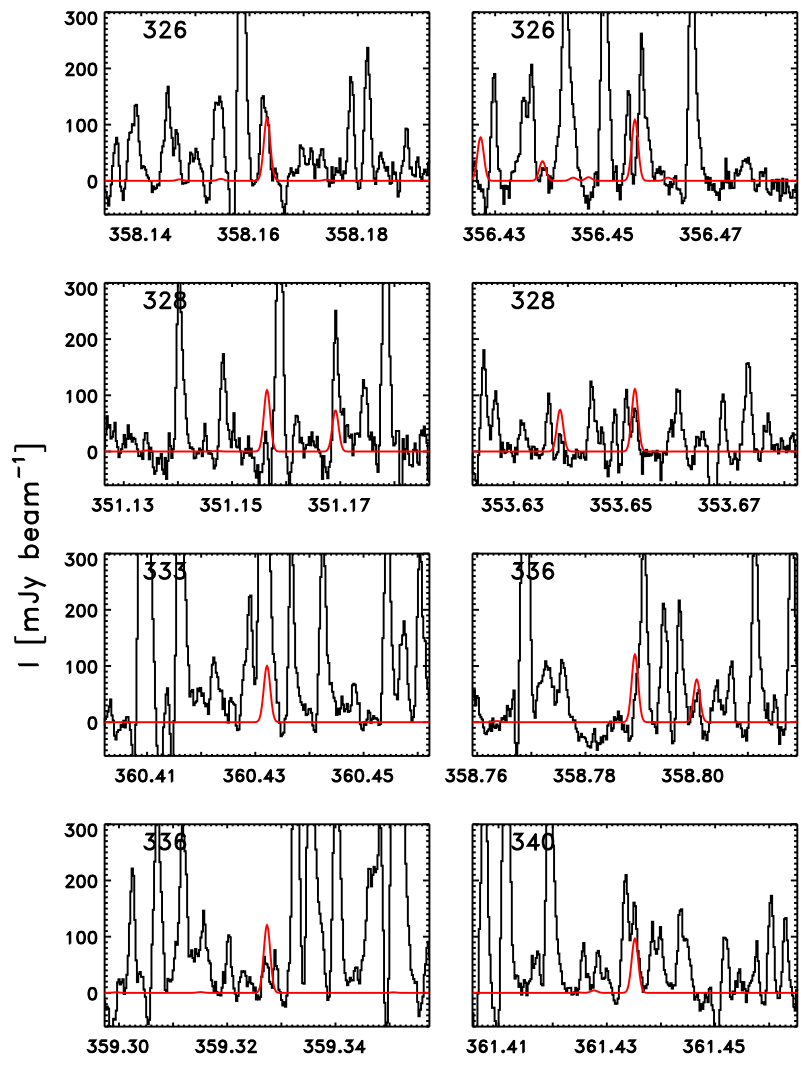

Freq. $[\mathrm{GHz}]$
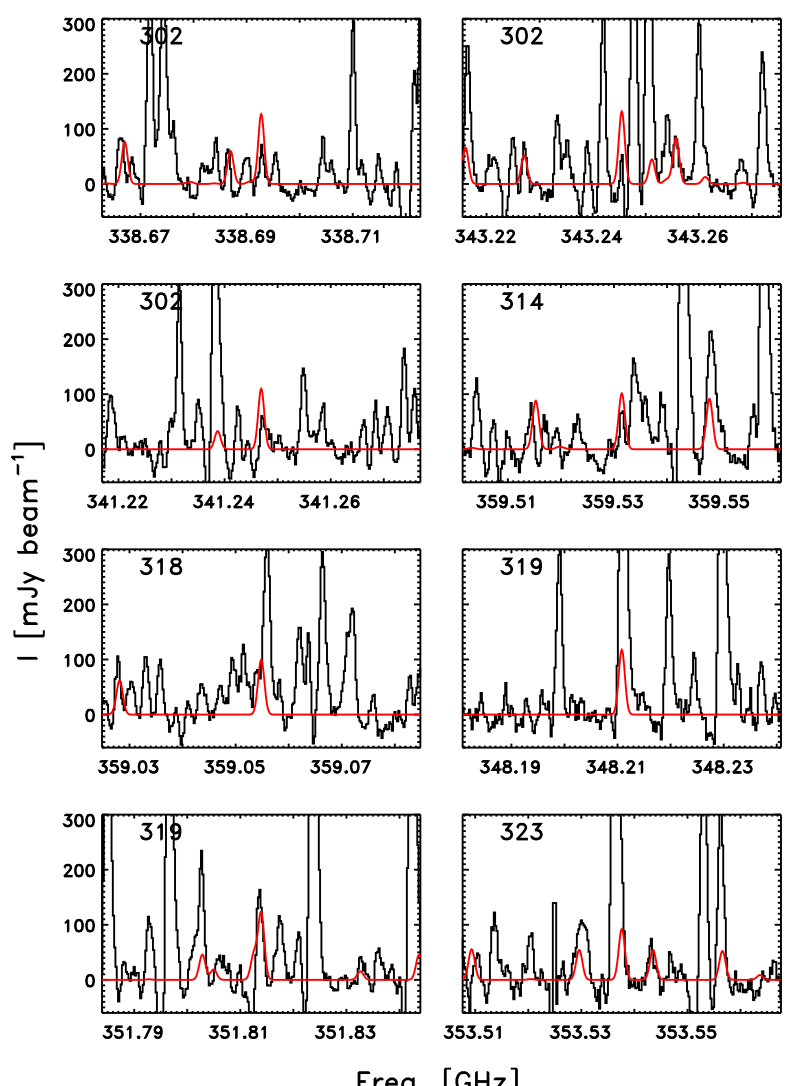

Freq. $[\mathrm{GHz}]$

Fig. C.4. As in Fig. C.1 for the 24 brightest lines of $g G g^{\prime}$ ethylene glycol with $\tau<0.2$ as expected from the synthetic spectrum. 


\section{C.5. Acetic acid}
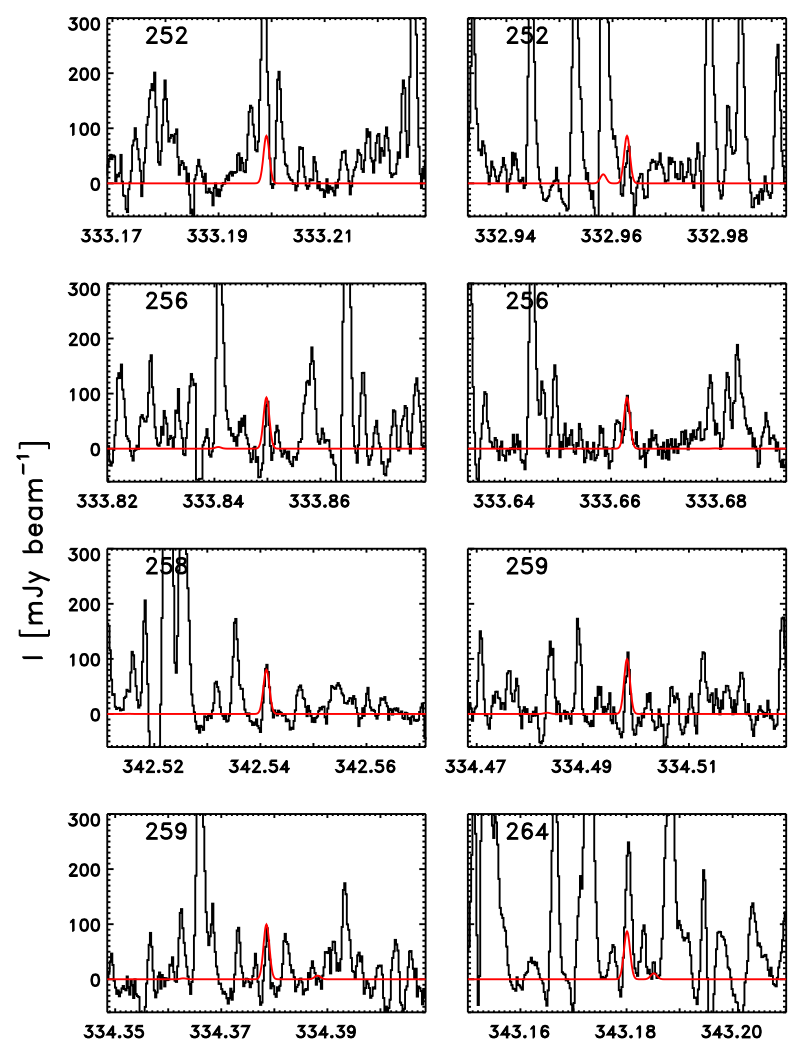

Freq. [GHz]
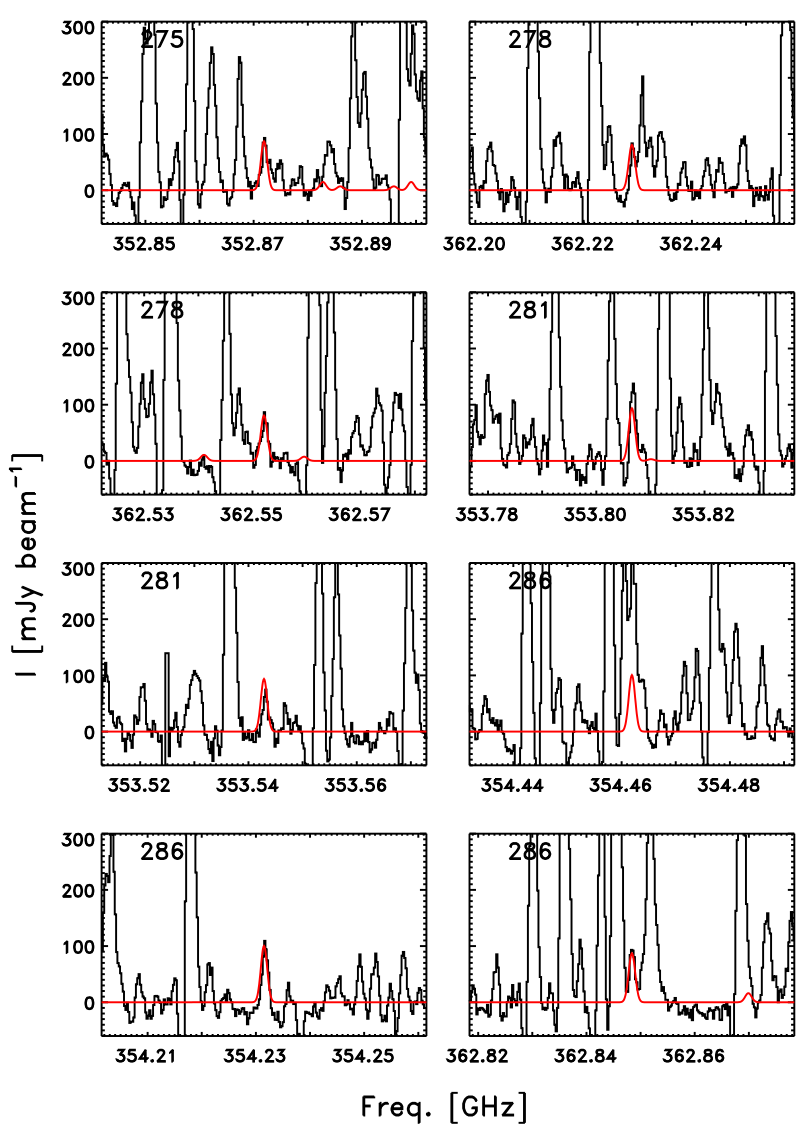

req. $[\mathrm{GHz}]$
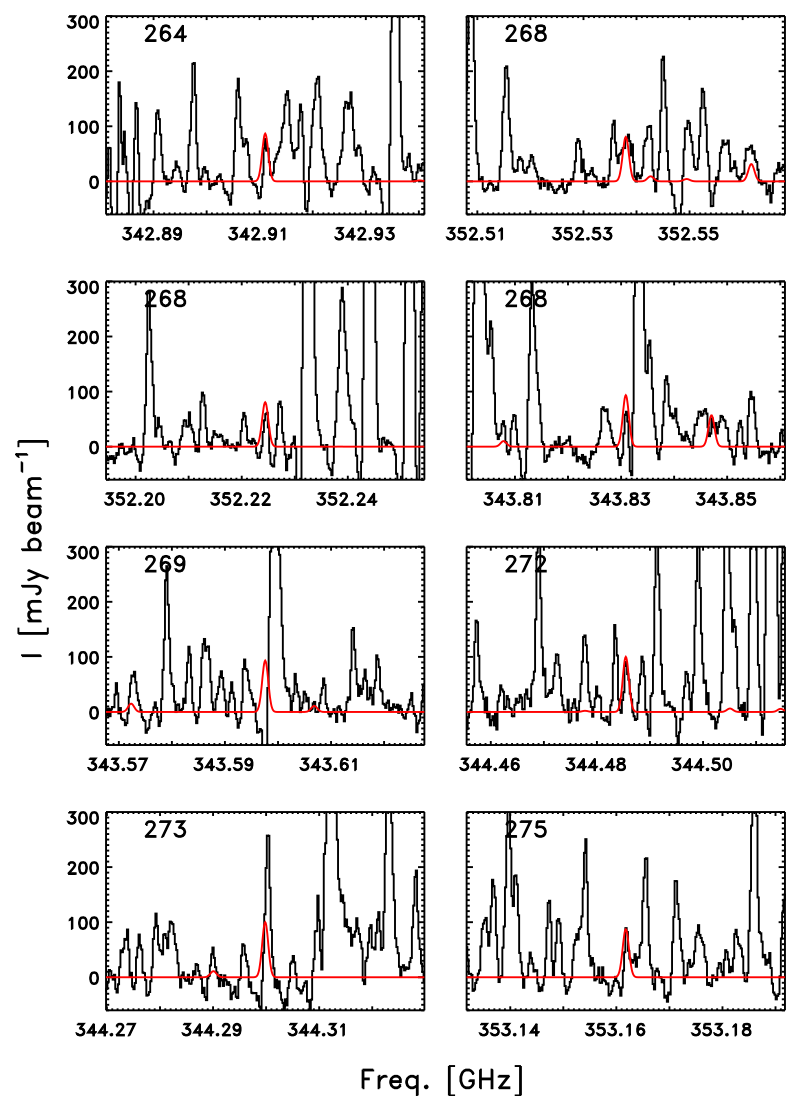

Freq. [GHz]

Fig. C.5. The 24 brightest lines of acetic acid with $\tau<0.2$ as expected from the synthetic spectrum sorted according to $E_{\text {up }}$. 
C.6. Glycolaldehyde: Deuterated isotopologues
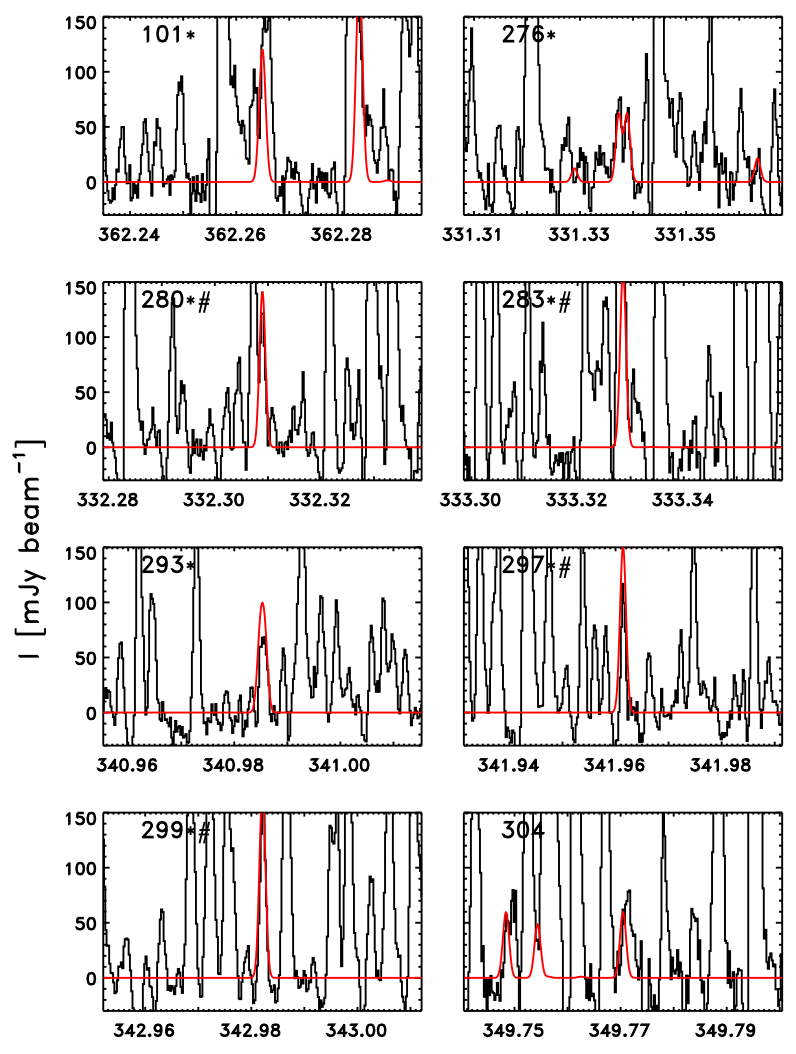

Freq. [GHz]
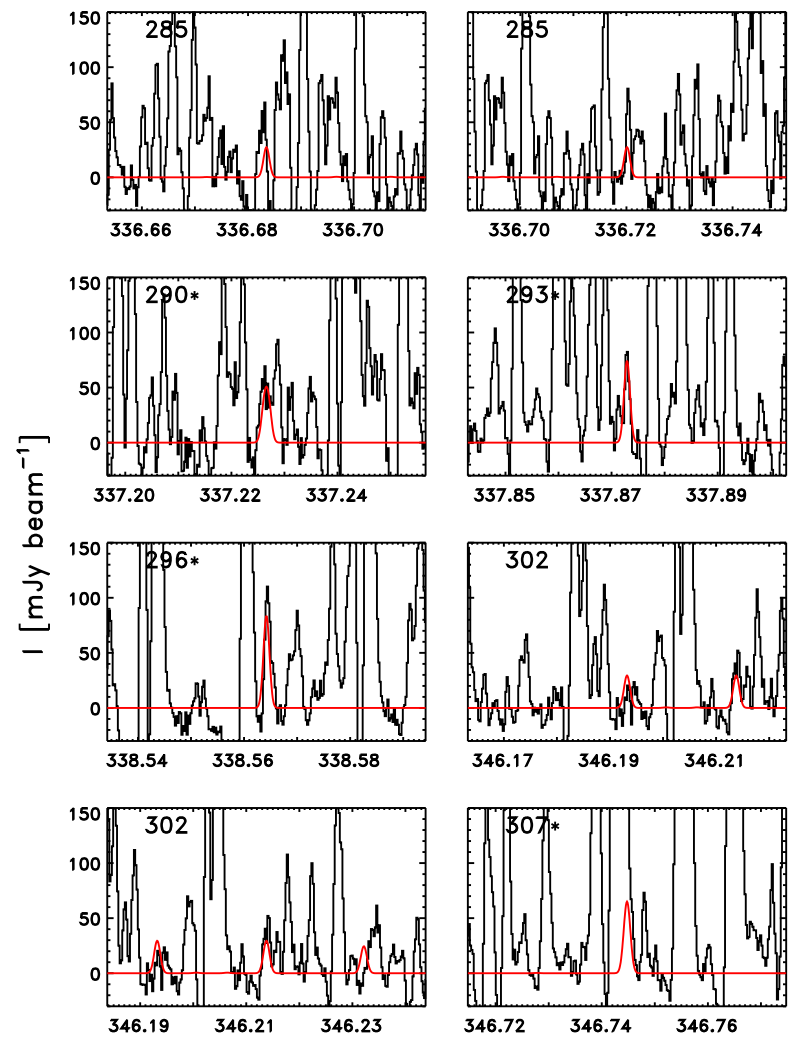

Freq. [GHz]
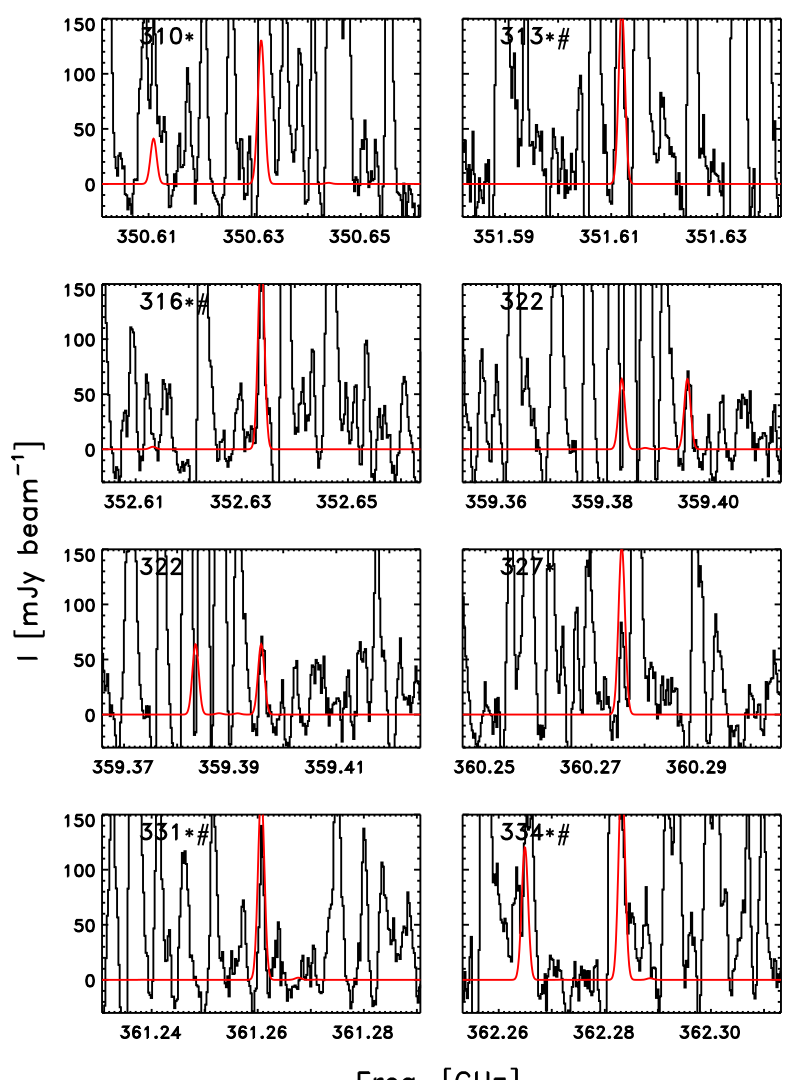

Freq. [GHz]
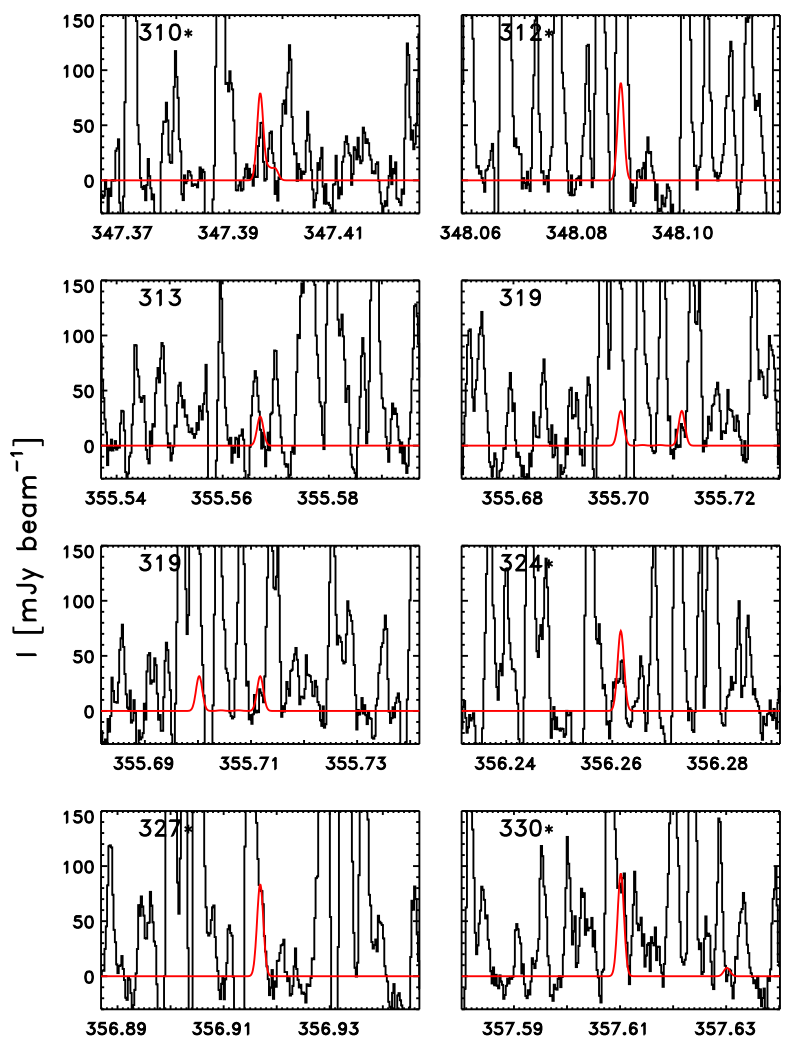

Freq. [GHz]

Fig. C.6. Detection $\mathrm{CHDOHCHO}$ (upper panels) and $\mathrm{CH}_{2} \mathrm{OHCDO}$ (lower panels) showing the 16 brightest lines as expected from the synthetic spectrum. 

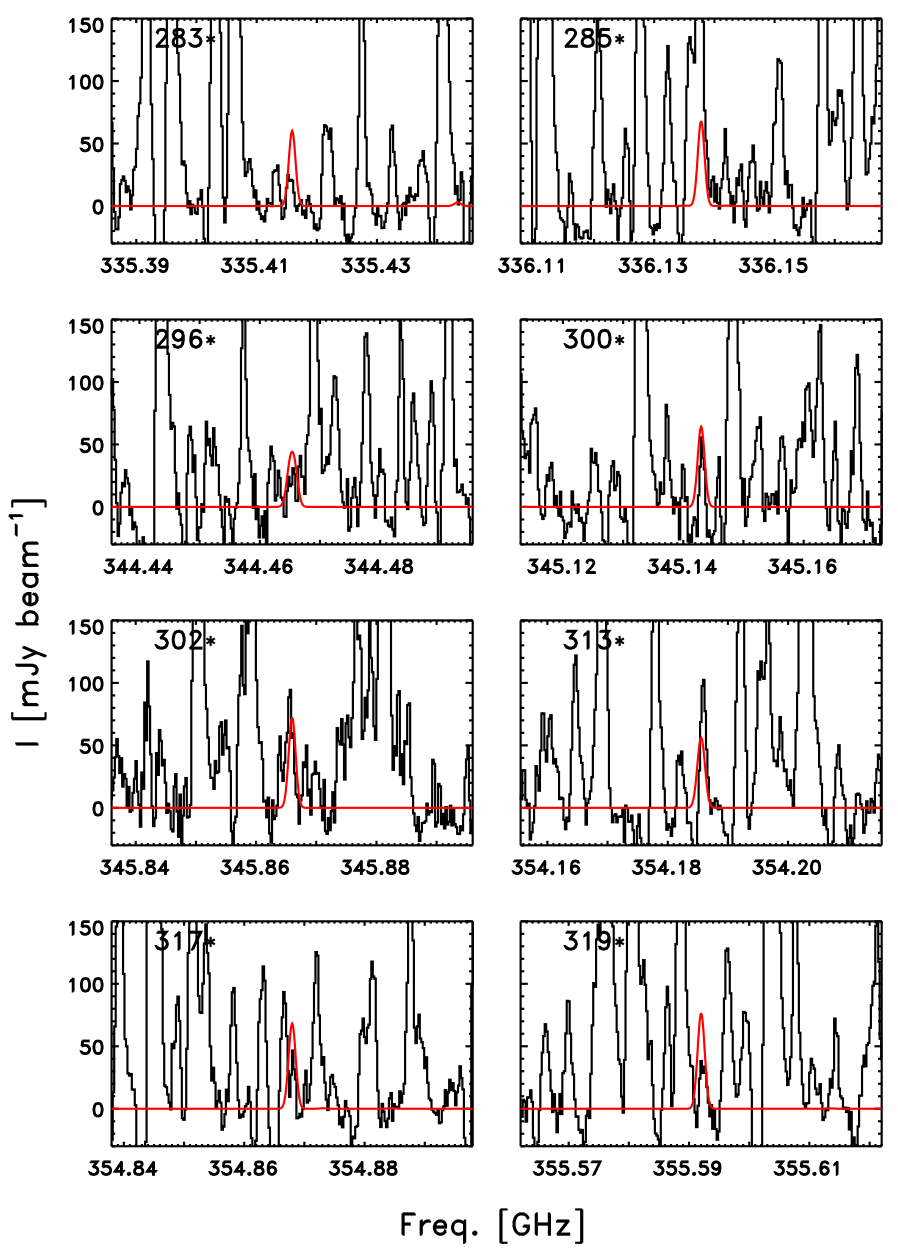

Fig. C.7. Detection of $\mathrm{CH}_{2} \mathrm{ODCHO}$ showing the eight brightest lines as expected from the synthetic spectrum. 
C.7. Glycolaldehyde: ${ }^{13} \mathrm{C}$-isotopologues
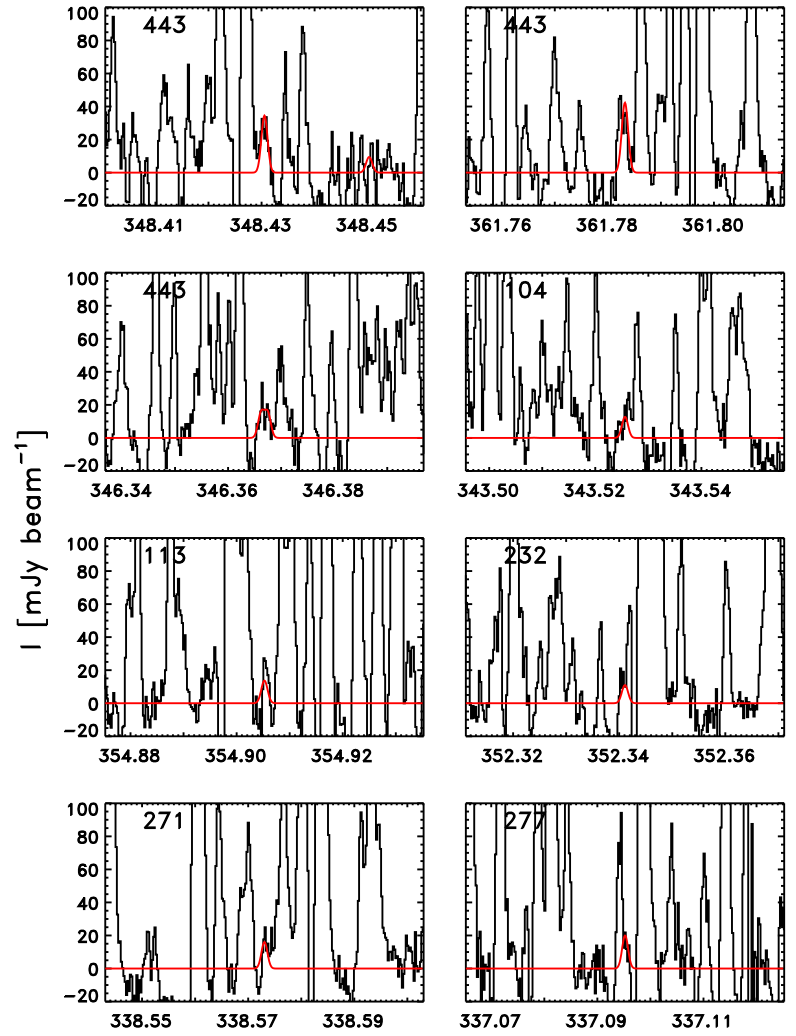

Freq. $[\mathrm{GHz}]$
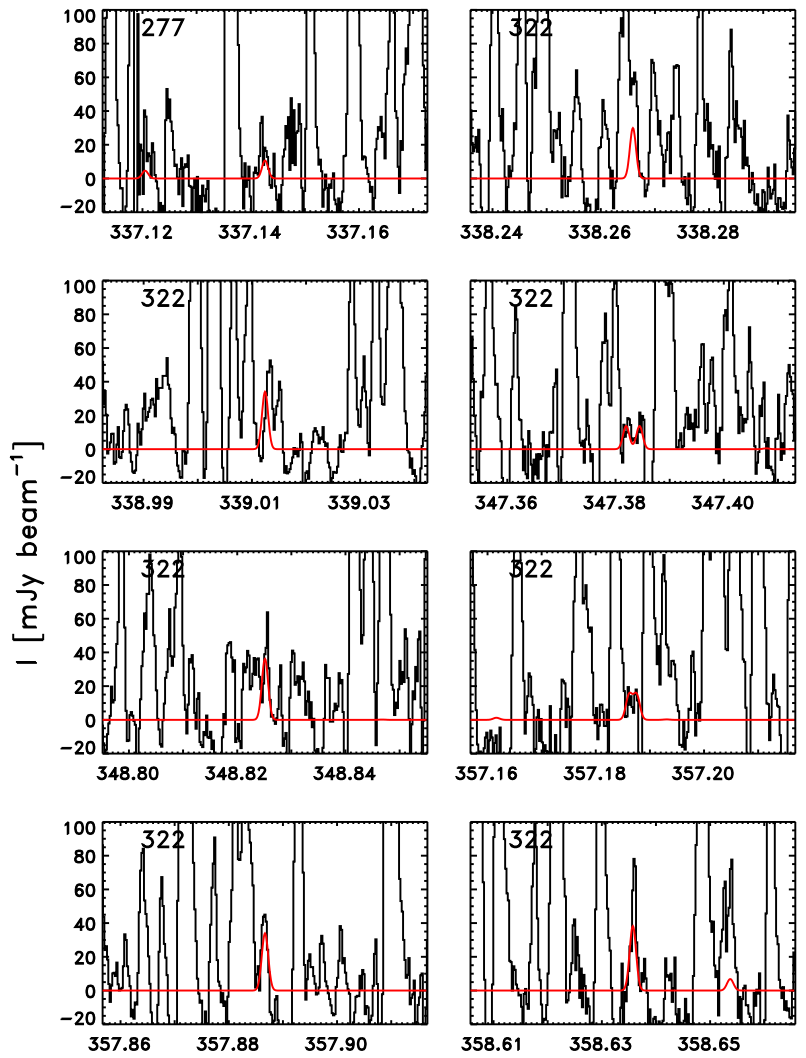

Freq. $[\mathrm{GHz}]$
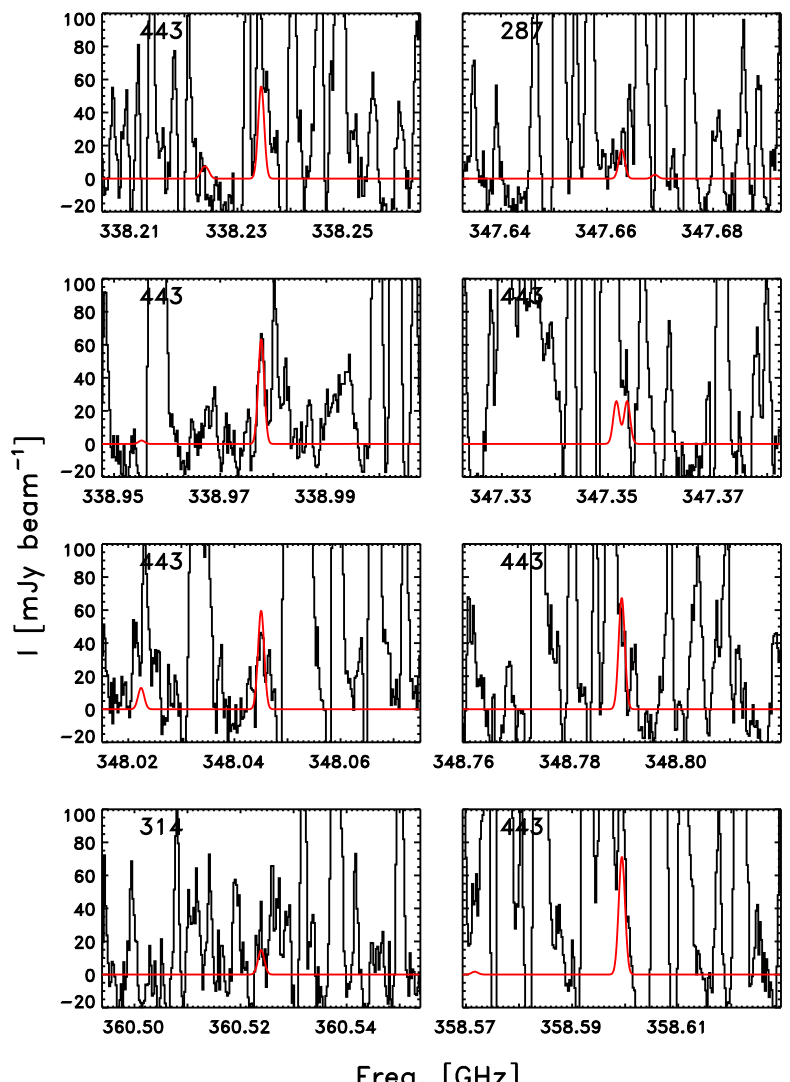

Freq. $[\mathrm{GHz}]$

Fig. C.8. Detection of the ${ }^{13} \mathrm{C}$ isotopologues of glycolaldehyde. The upper panels show the 16 best (bright/relatively well-isolated) lines of ${ }^{13} \mathrm{CH}_{2} \mathrm{OHCHO}$ and the lower panels the 8 best lines of $\mathrm{CH}_{2} \mathrm{OH}^{13} \mathrm{CHO}$. 